Supporting Information

\title{
Diol-Ritter Reaction: Regio- and Stereoselective Synthesis of Protected Vicinal-Aminoalcohols and Mechanistic Aspects of Diol Monoester Disproportionation
}

Mark E. Ondari, ${ }^{1}$ Jerzy Klosin, ${ }^{2}$ William R. Kruper, ${ }^{1}$ Ivan Lysenko ${ }^{3}$, Pulikkottil J. Thomas ${ }^{3}$, Kevin Cheng ${ }^{3}$, Khalil A. Abboud ${ }^{4}$ and William J. Kruper ${ }^{3 *}$

${ }^{1}$ Corteva Agriscience, 1710 Building, Midland, MI, 48674

${ }^{2}$ Corporate R\&D, The Dow Chemical Company, 1776 building, Midland, MI 48674

${ }^{3}$ Michigan State University, St Andrews, Midland MI, 48674

${ }^{4}$ Department of Chemistry, University of Florida, Gainesville, Florida, 32611

kruperw2@msu.edu

\section{Table of Content}

NMR Spectra:

HPLC Separation Data:

DSC Data:

X-ray Data:
S1-S60

S61-S62

S63-S64

S65-S73 
Figure S1: ${ }^{1} \mathrm{H}$ NMR spectrum of 1-acetamido-3-chloropropan-2-yl acetate ((rac)-4a) $\left(\mathrm{CDCl}_{3}, 300 \mathrm{MHz}\right)$.

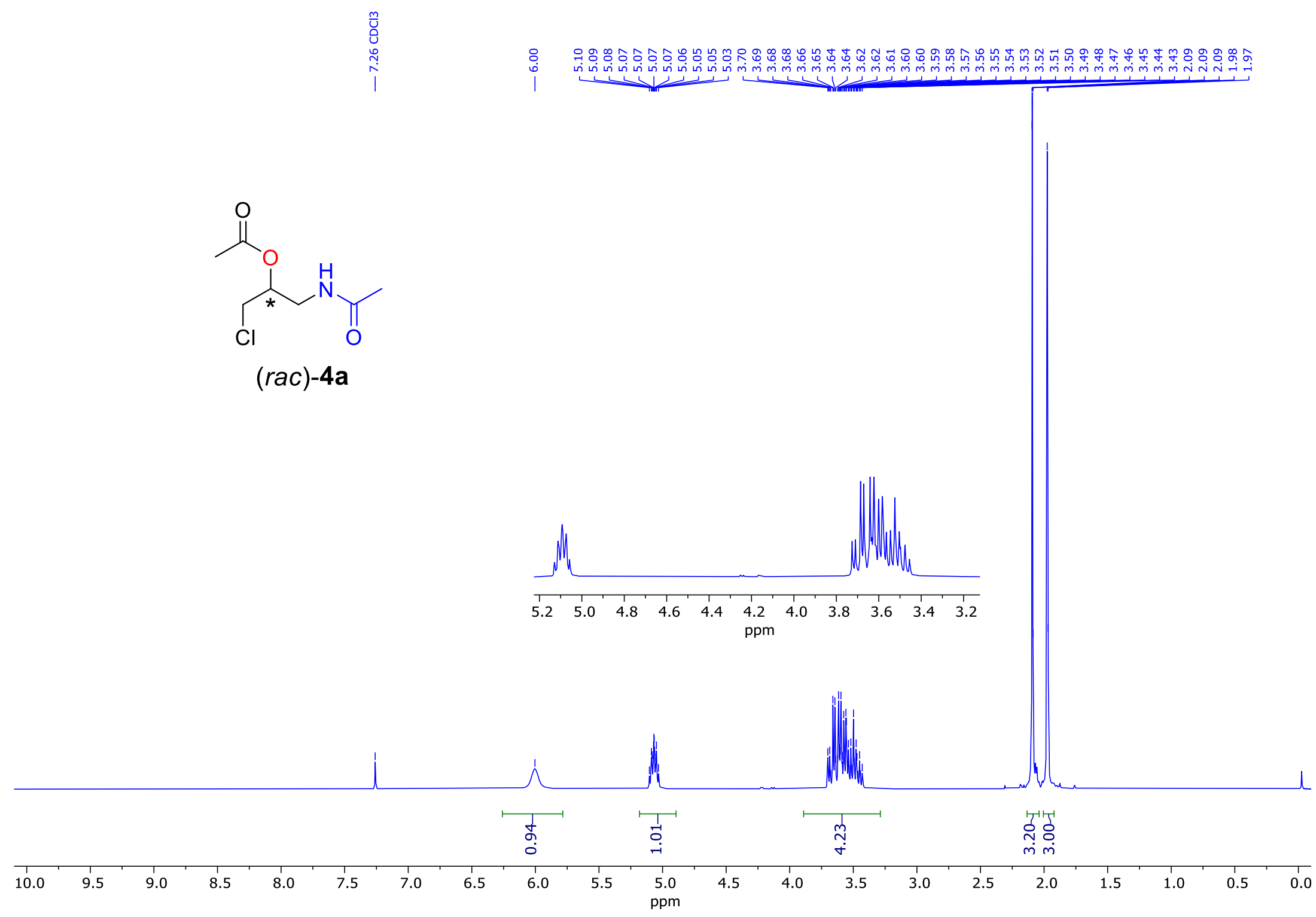


Figure S2: ${ }^{13} \mathrm{C}\left\{{ }^{1} \mathrm{H}\right\}$ NMR spectrum of 1-acetamido-3-chloropropan-2-yl acetate ((rac)-4a) $\left(\mathrm{CDCl}_{3}, 75 \mathrm{MHz}\right)$.

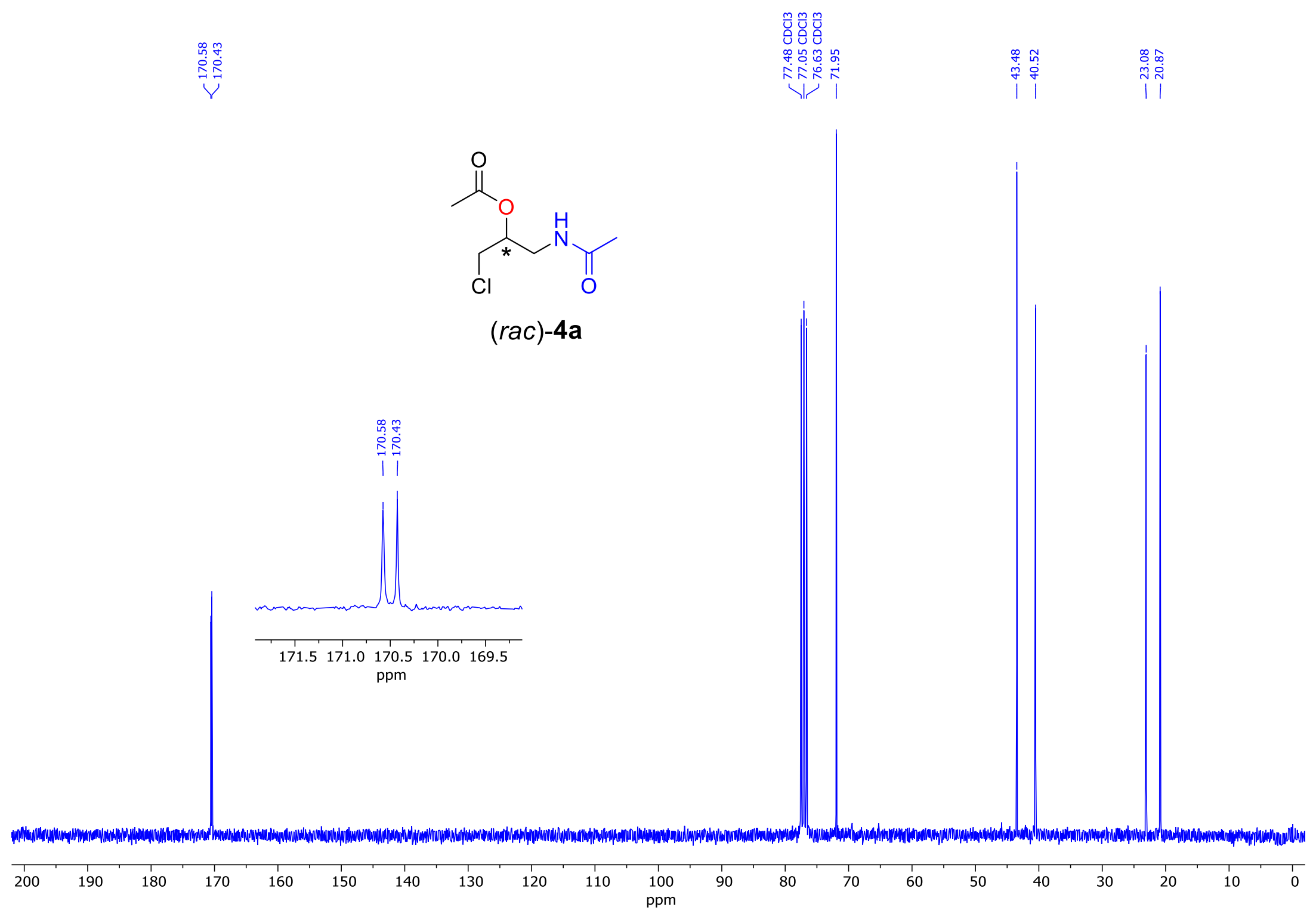


Figure S3: ${ }^{1} \mathrm{H}$ NMR spectrum of 1-acetamido-3-chloropropan-2-yl acetate $((R)-4 \mathbf{c})\left(\mathrm{CDCl}_{3}, 300 \mathrm{MHz}\right)$.

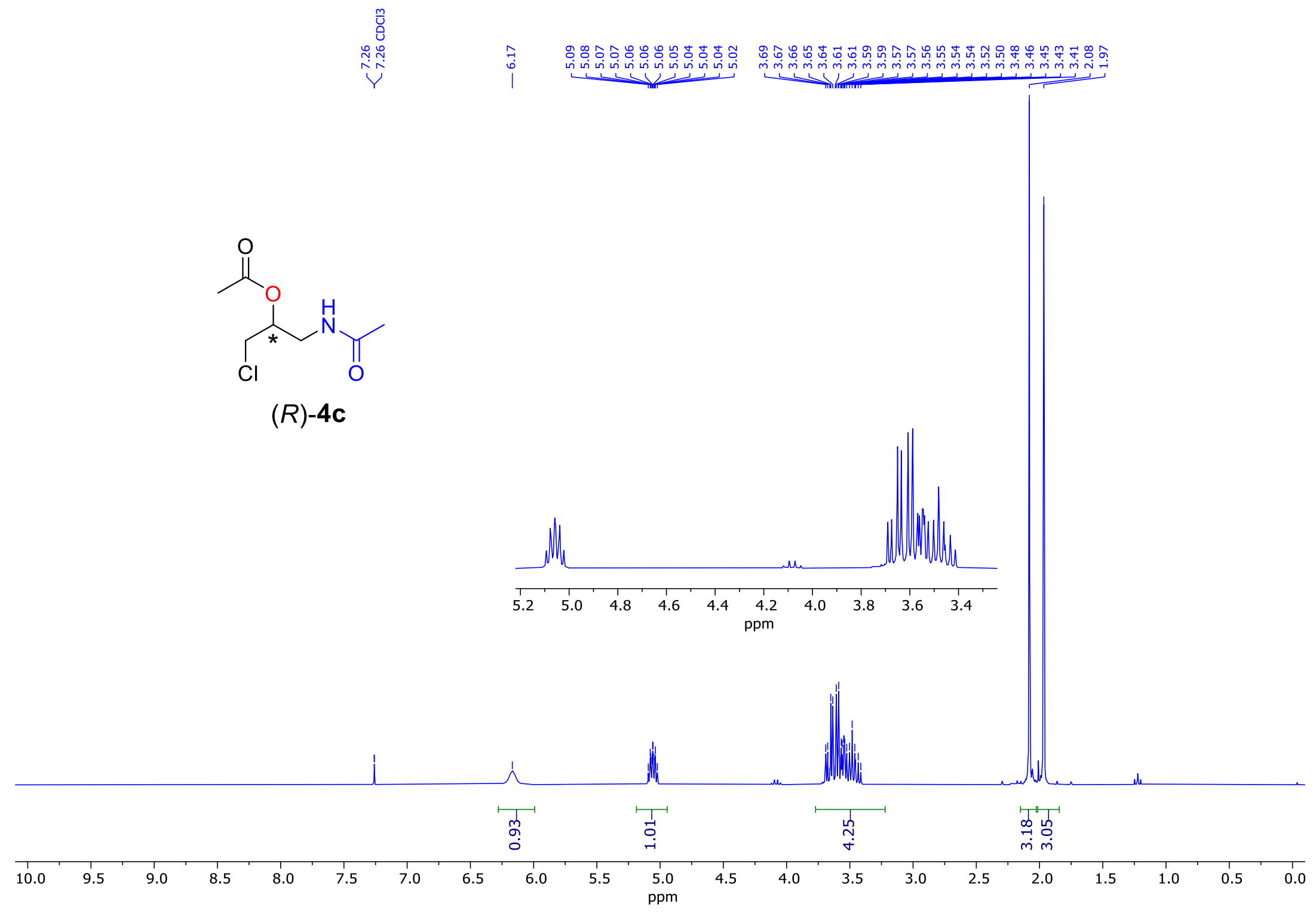


Figure S4: ${ }^{1} \mathrm{H}$ NMR spectrum of 1-acetamido-3-chloropropan-2-yl acetate $((S)-\mathbf{4 b})\left(\mathrm{CDCl}_{3}, 300 \mathrm{MHz}\right)$.

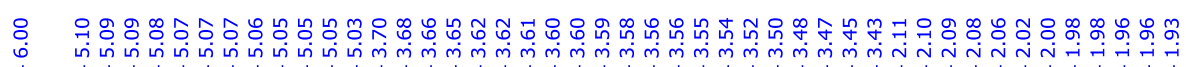

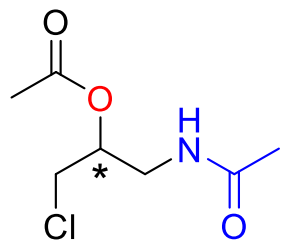

(S)-4b

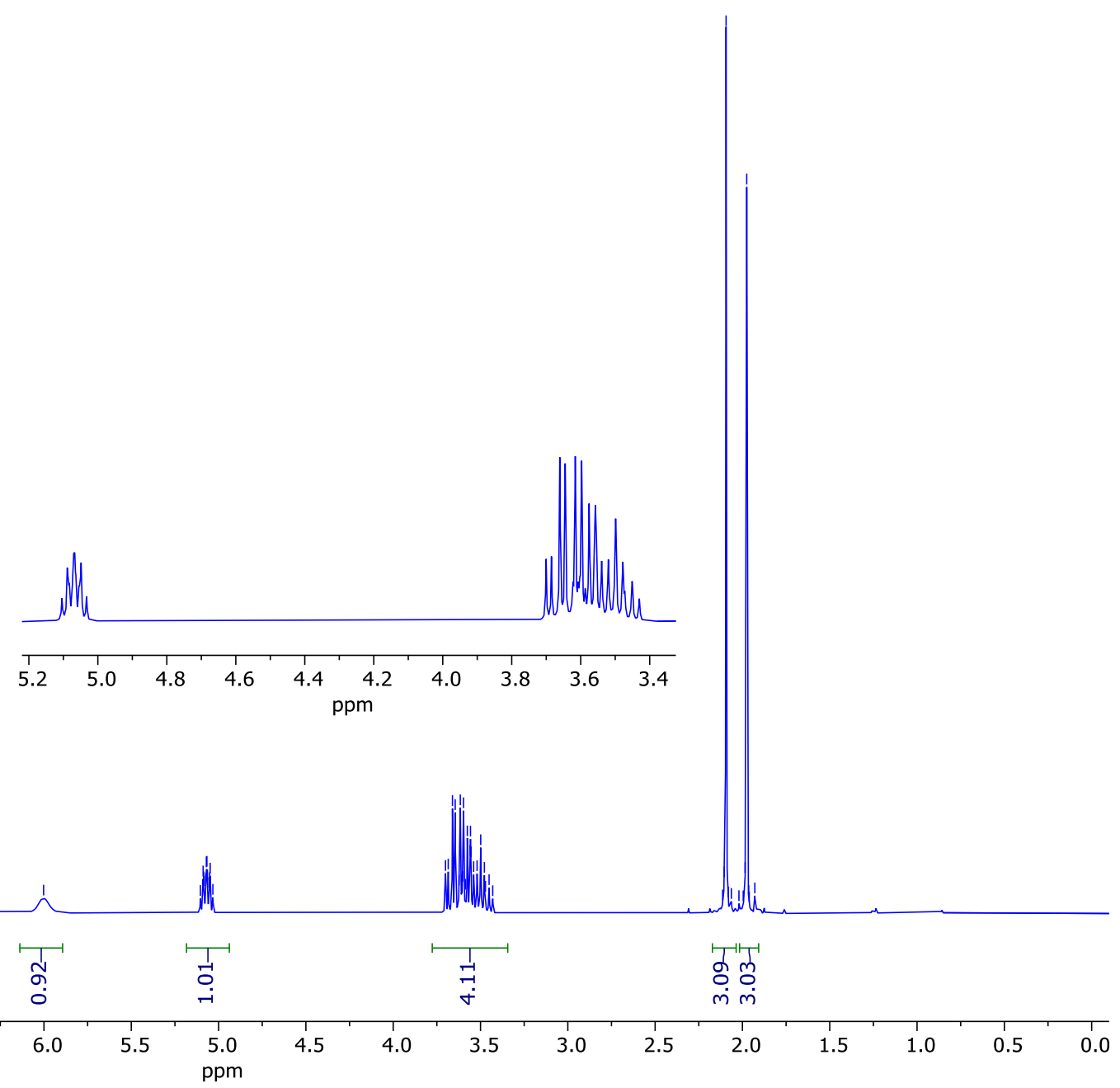


Figure S5: ${ }^{13} \mathrm{C}\left\{{ }^{1} \mathrm{H}\right\}$ NMR spectrum of 1 -acetamido-3-chloropropan-2-yl acetate $((S)-\mathbf{4 b})\left(\mathrm{CDCl}_{3}, 75 \mathrm{MHz}\right)$.

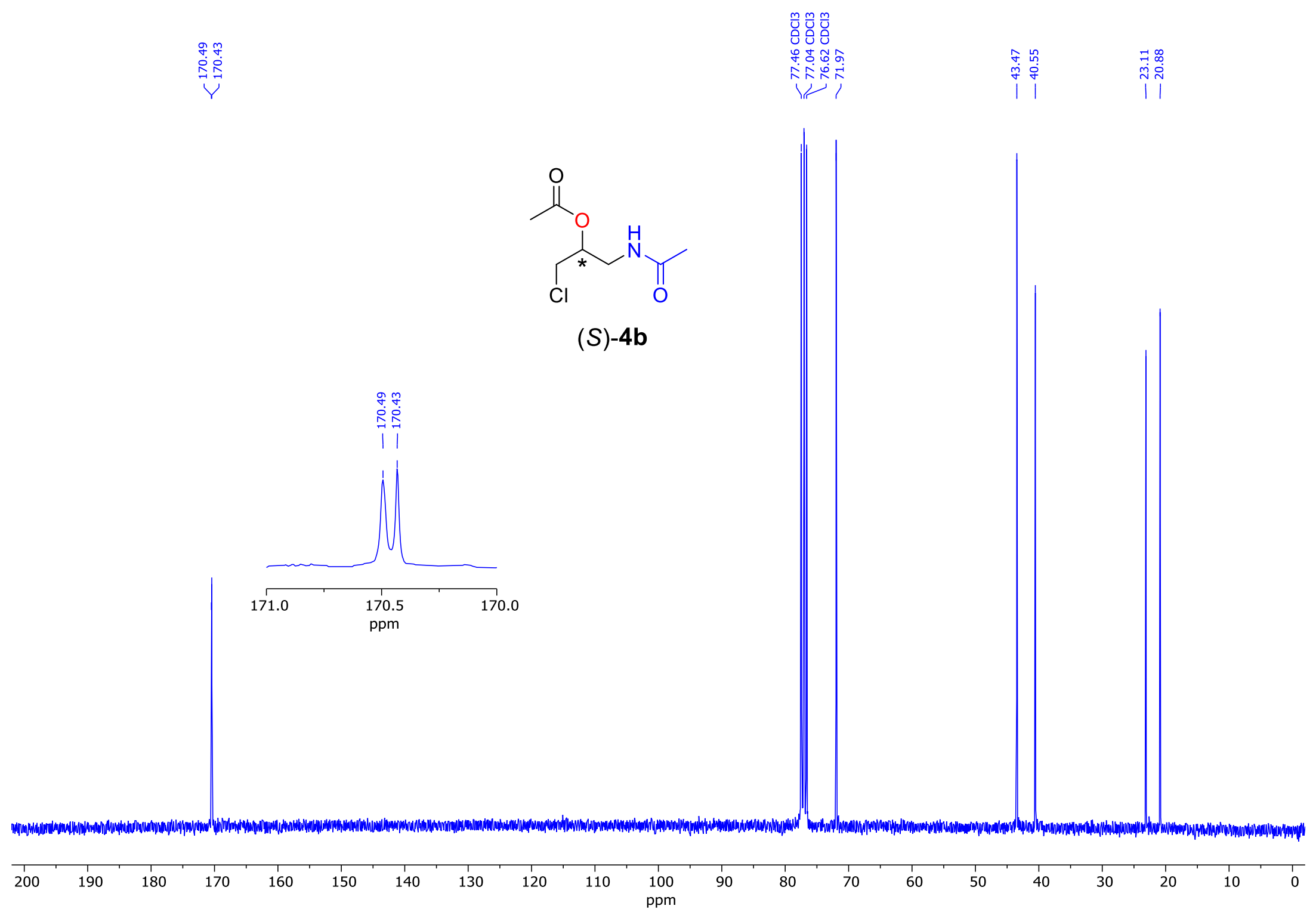


Figure S6: HSQC NMR spectrum of 1-acetamido-3-chloropropan-2-yl acetate $((S)-\mathbf{4 b})\left(\mathrm{CDCl}_{3},\right)$.

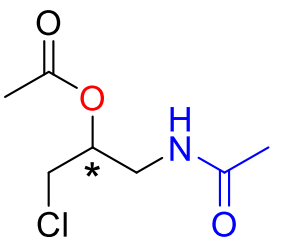

(S)-4b

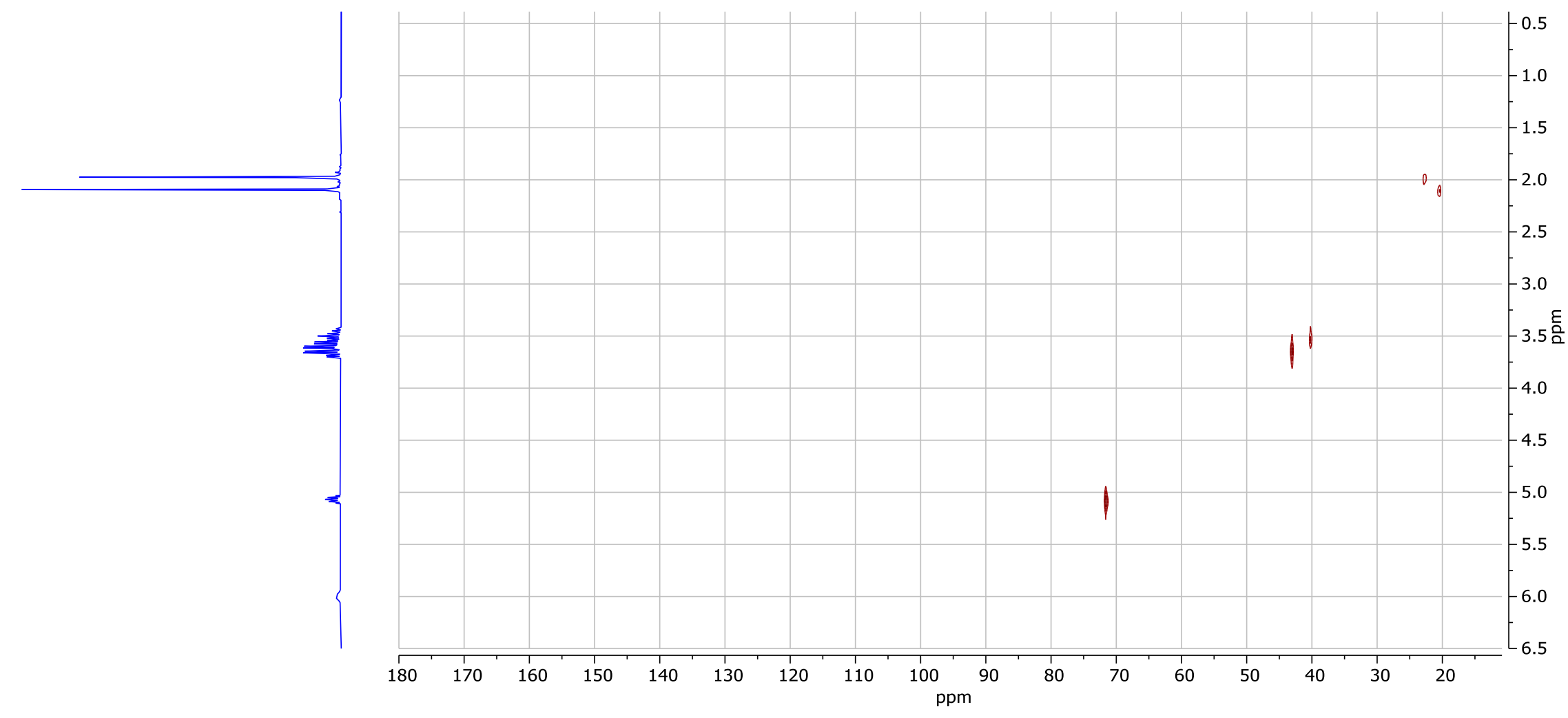


Figure S7: ${ }^{1} \mathrm{H}$ NMR spectrum of 1-(acetamido-2,2,2-d 3 )-3-chloropropan-2-yl acetate ( $\left.(r a c)-4 d\right)\left(\mathrm{CDCl}_{3}, 300 \mathrm{MHz}\right)$.

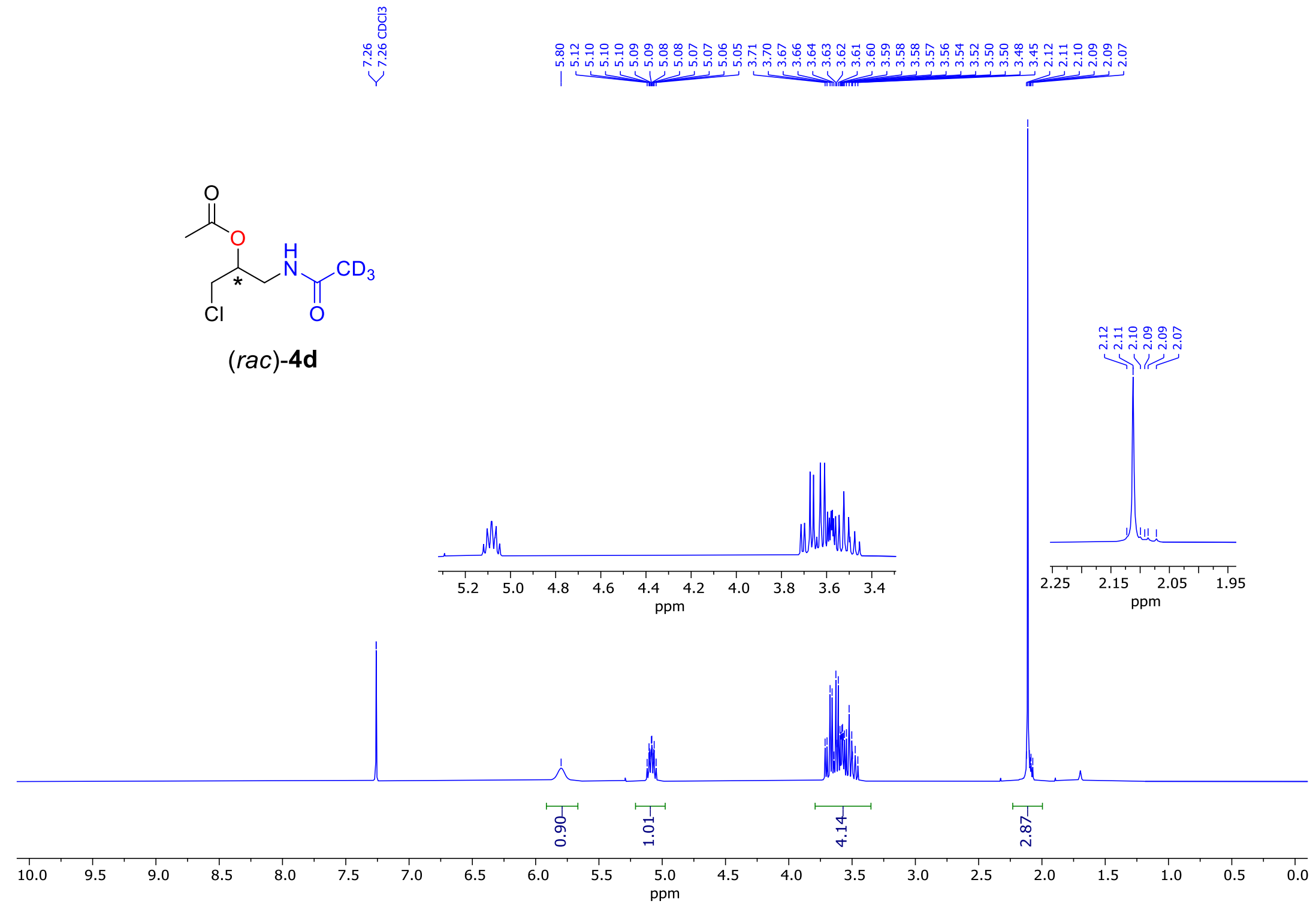


Figure S8: ${ }^{13} \mathrm{C}\left\{{ }^{1} \mathrm{H}\right\}$ NMR spectrum of 1-(acetamido-2,2,2-d 3$)-3$-chloropropan-2-yl acetate ((rac-4d) $\left(\mathrm{CDCl}_{3}, 75 \mathrm{MHz}\right)$.

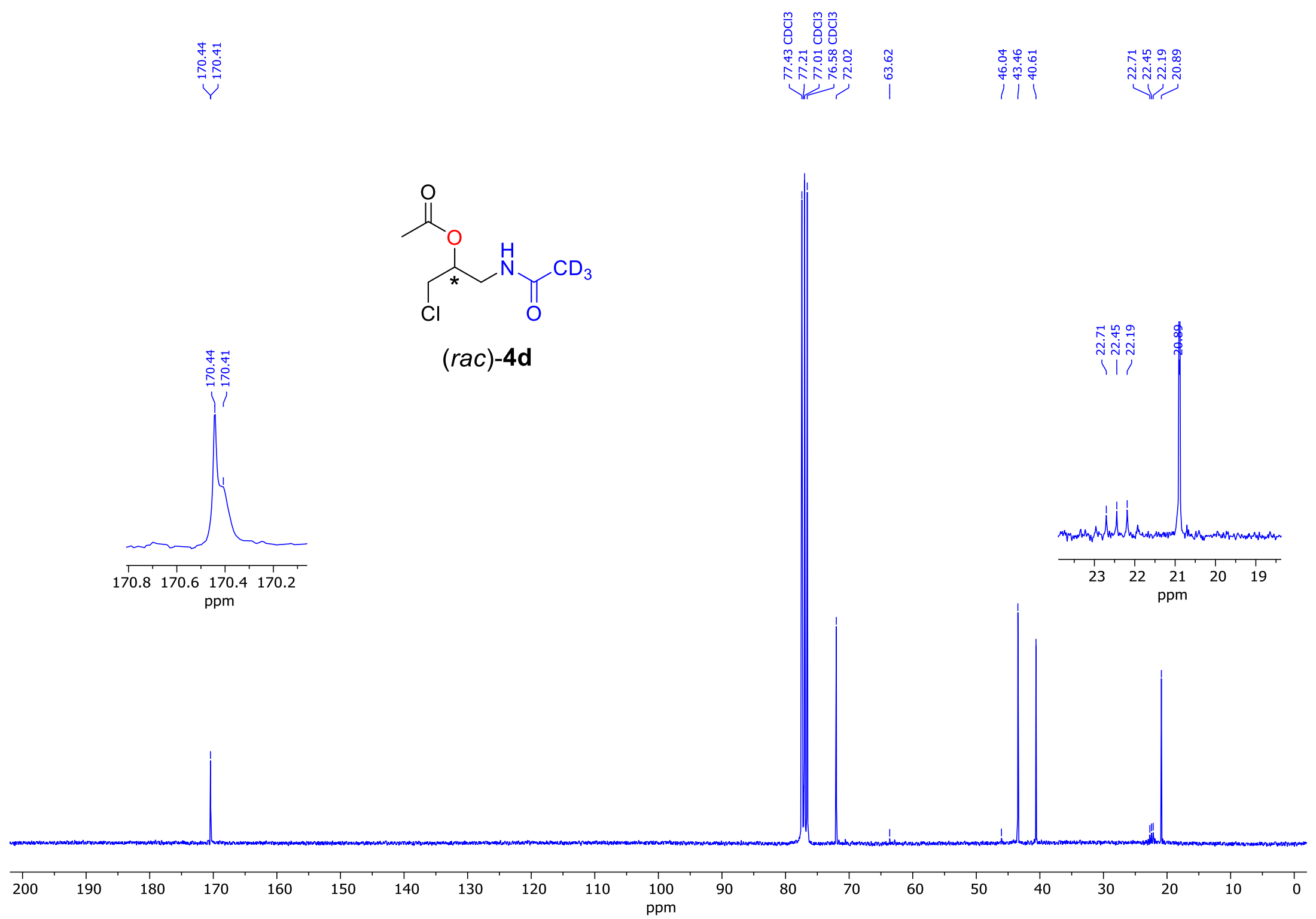


Figure S9: (A): ${ }^{1} \mathrm{H}$ NMR spectrum of amidoester 4 in $\mathrm{CDCl}_{3}$; (B): with 1.0 eq $\mathrm{BF}_{3}$ etherate; (C): with 1.1 eq $\mathrm{KF}$ extraction to $\mathrm{BF}_{3}-$ free $4\left(\mathrm{CDCl}_{3}\right.$, $300 \mathrm{MHz}$ ).

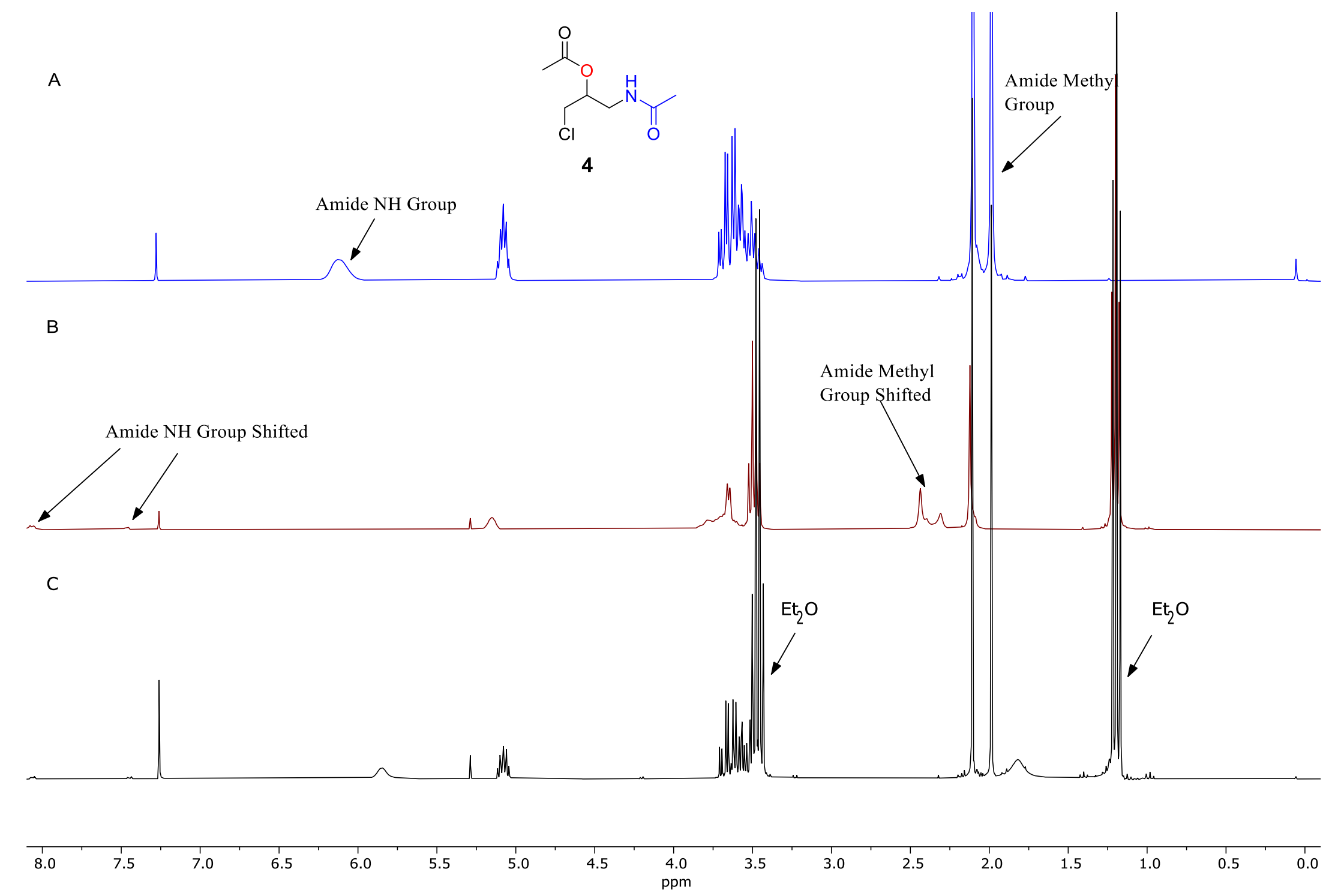


Figure S10: ${ }^{1} \mathrm{H}$ NMR spectrum of 1-acetamido-3-chloropropan-2-yl benzoate $((R)-6 \mathbf{b})\left(\mathrm{CDCl}_{3}, 300 \mathrm{MHz}\right)$.

$$
\text { Uับ }
$$

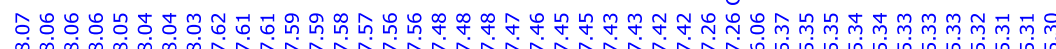

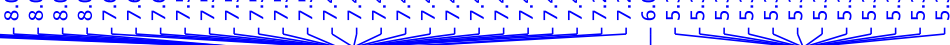

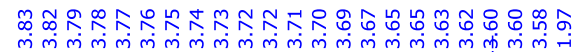
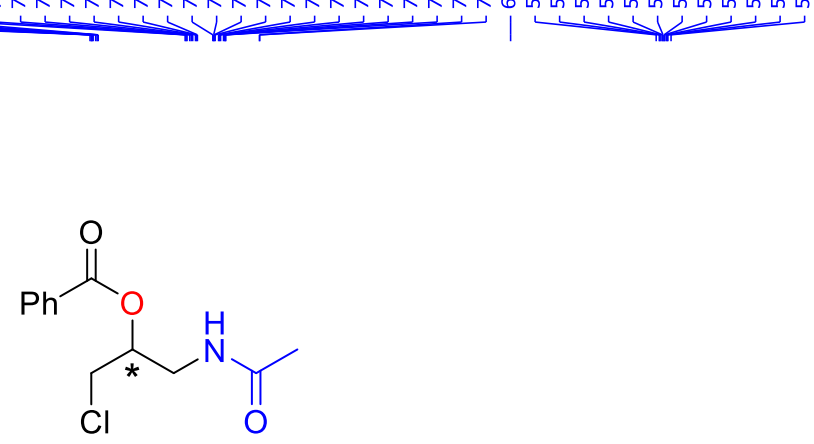

$(R)-6 \mathbf{b}$

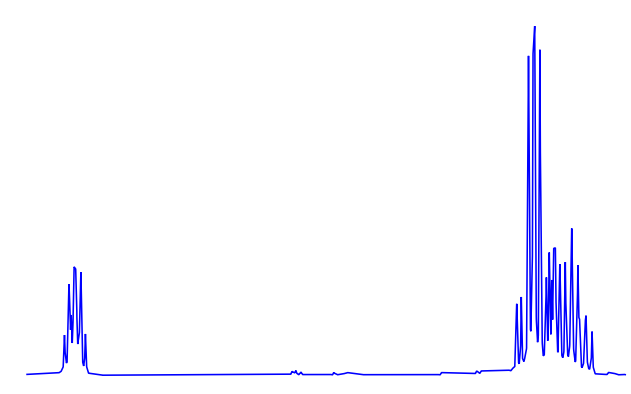

$\begin{array}{llllllllllll}5.4 & 5.2 & 5.0 & 4.8 & 4.6 & 4.4 & 4.2 & 4.0 & 3.8 & 3.6\end{array}$
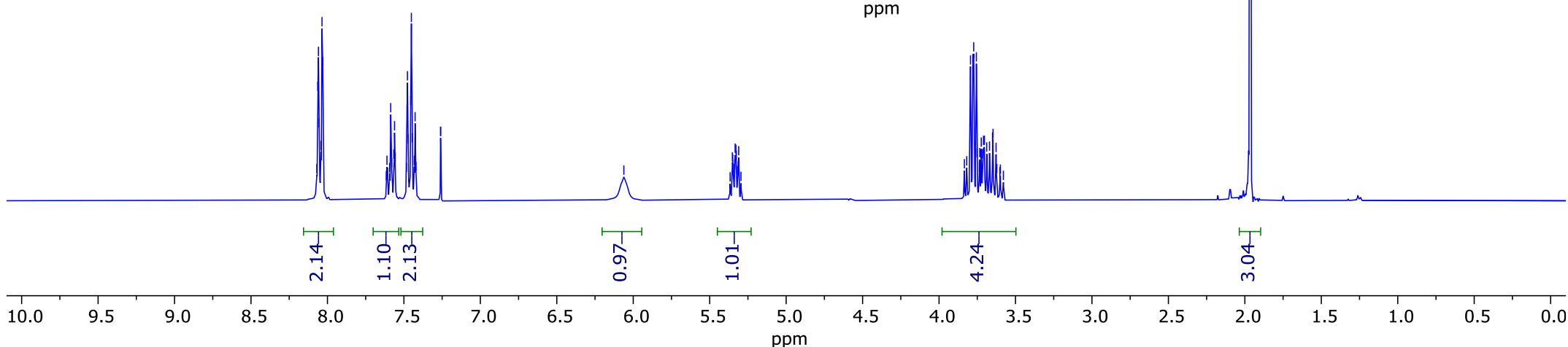
Figure S11: ${ }^{13} \mathrm{C}\left\{{ }^{1} \mathrm{H}\right\}$ NMR spectrum of 1-acetamido-3-chloropropan-2-yl benzoate $((R)-6 \mathbf{b})\left(\mathrm{CDCl}_{3}, 75 \mathrm{MHz}\right)$.

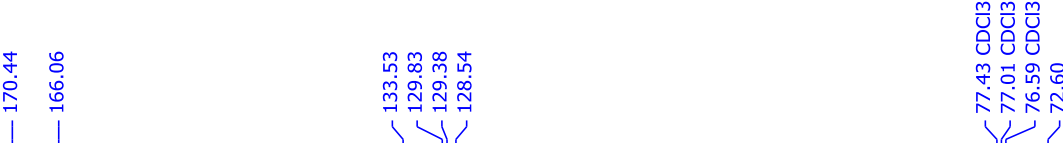

1

i

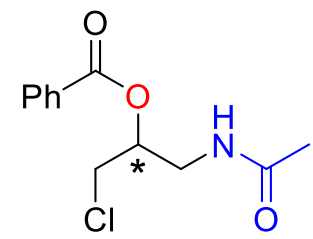

$(R)-6 \mathbf{b}$

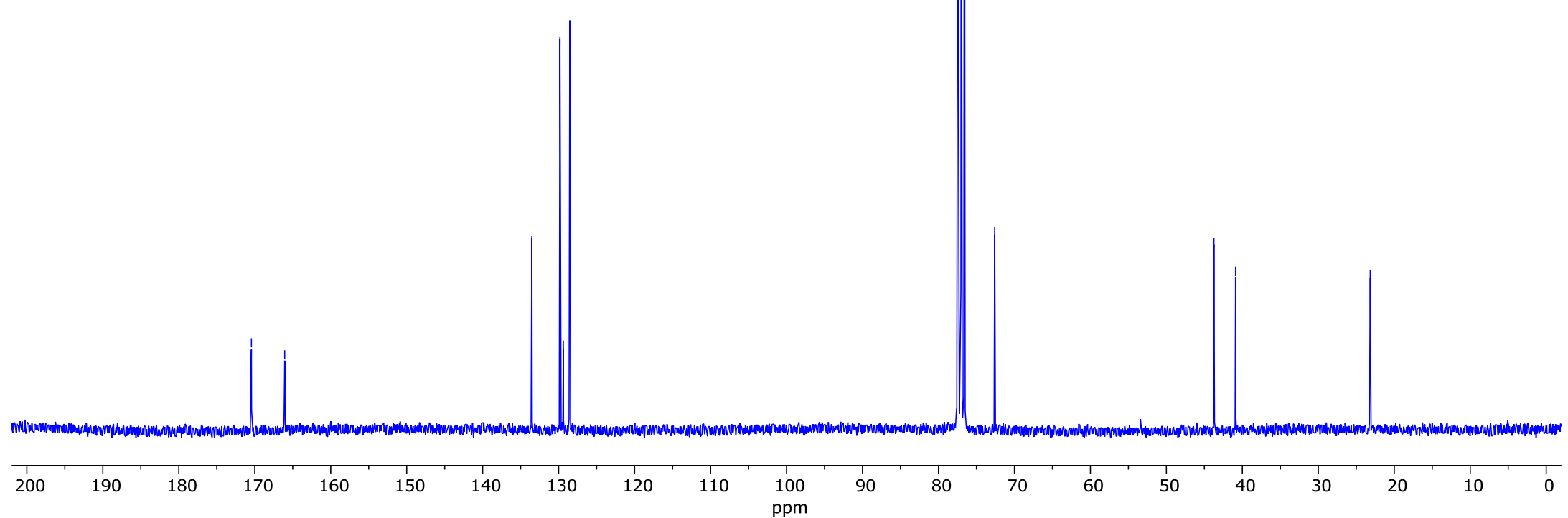


Figure S12: ${ }^{1} \mathrm{H}$ NMR spectrum of 1-acetamido-3-chloropropan-2-yl pivalate ( $\left.(\mathrm{rac})-\mathbf{8}\right)\left(\mathrm{CDCl}_{3}, 300 \mathrm{MHz}\right)$.

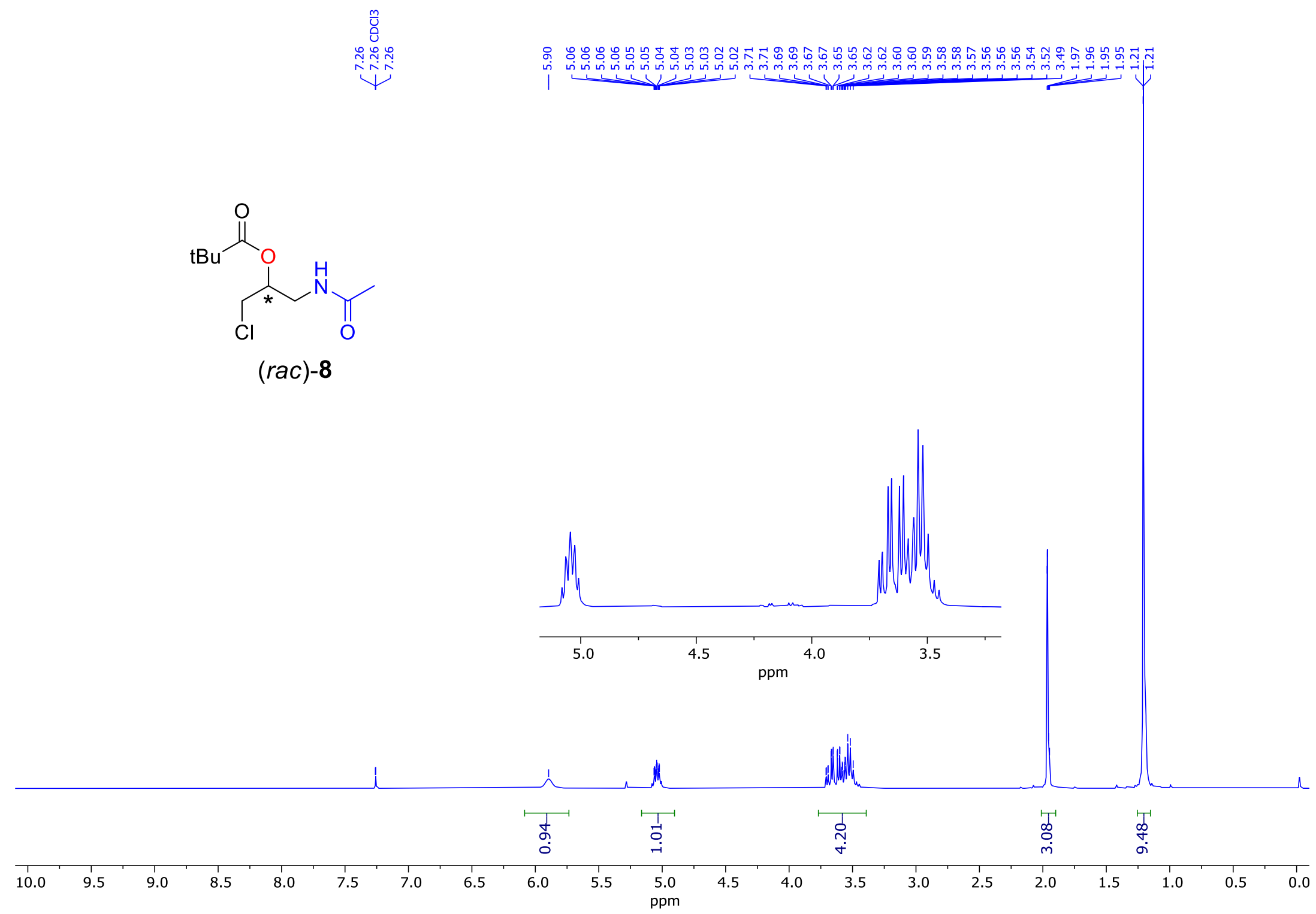


Figure S13: ${ }^{13} \mathrm{C}\left\{{ }^{1} \mathrm{H}\right\}$ NMR spectrum of 1-acetamido-3-chloropropan-2-yl pivalate $((\mathrm{rac})-8)\left(\mathrm{CDCl}_{3}, 75 \mathrm{MHz}\right)$.

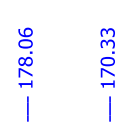
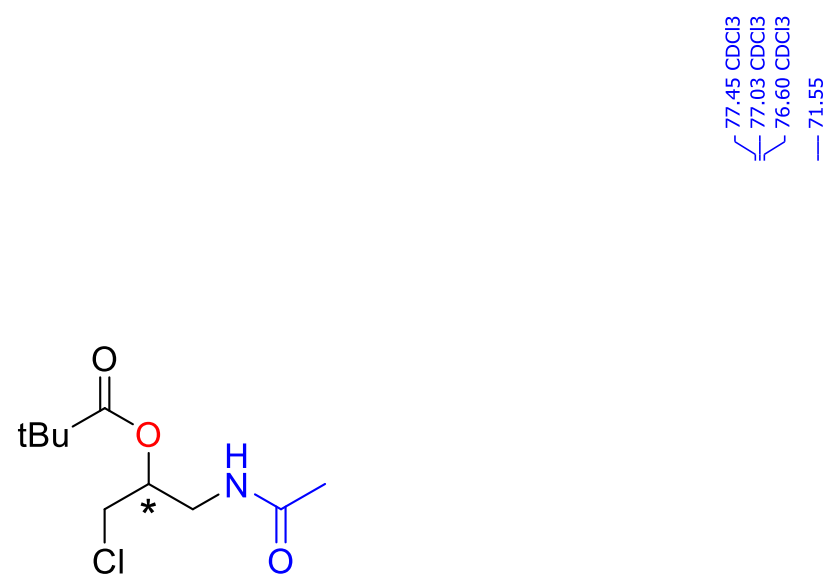

(rac)-8
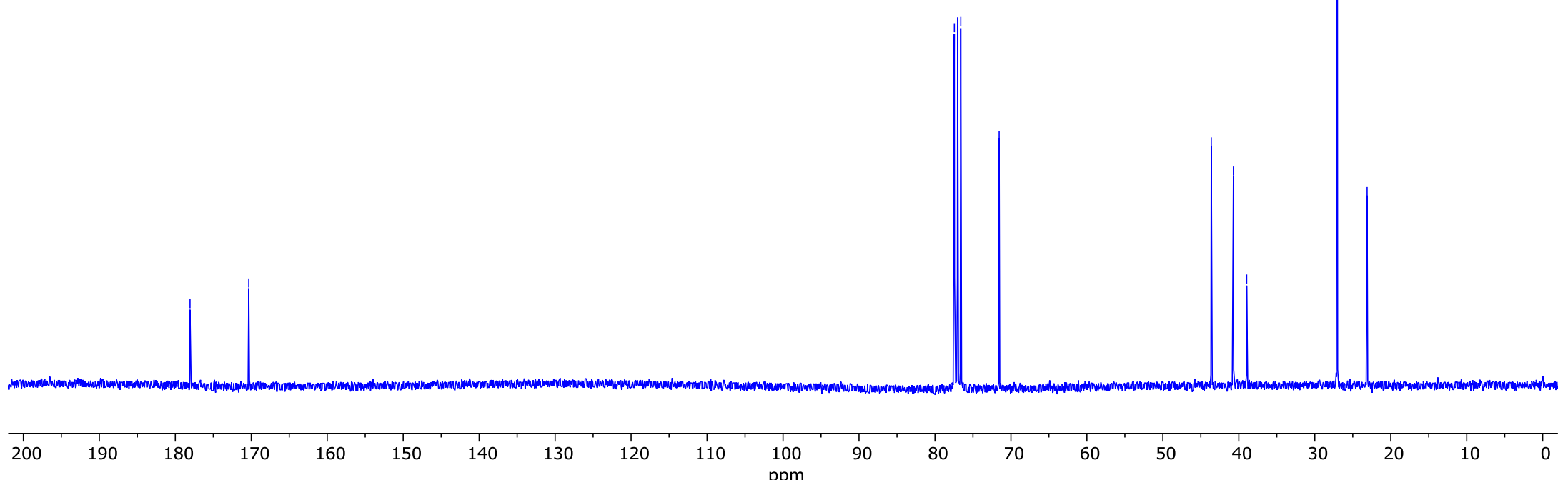
Figure S14: ${ }^{1} \mathrm{H}$ NMR spectrum of 1-acetamido-3-(1,3-dioxoisoindolin-2-yl)propan-2-yl acetate ((rac)-10a) $\left(\mathrm{CDCl}_{3}, 300 \mathrm{MHz}\right)$.

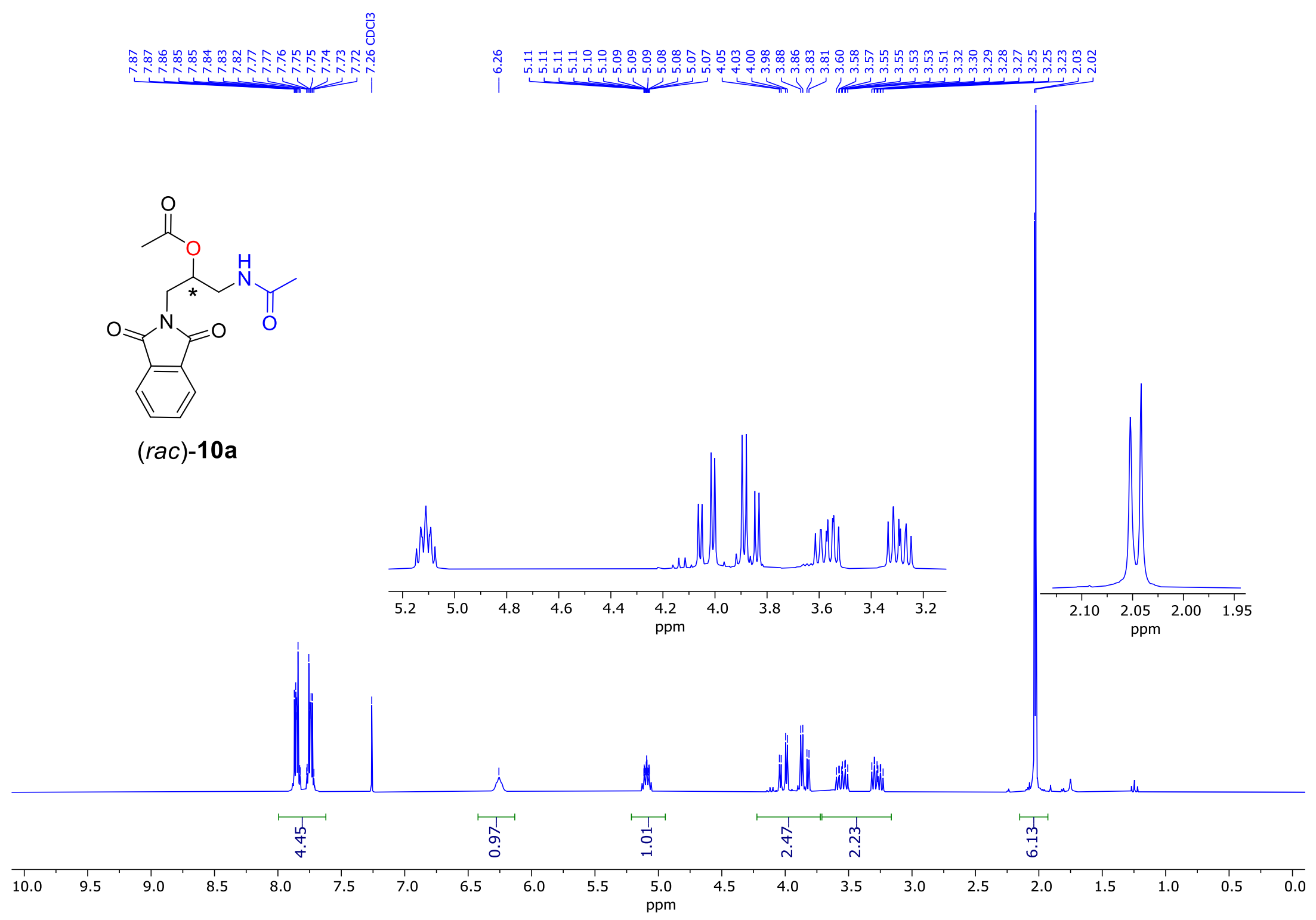


Figure S15: ${ }^{13} \mathrm{C}\left\{{ }^{1} \mathrm{H}\right\}$ NMR spectrum of 1-acetamido-3-(1,3-dioxoisoindolin-2-yl)propan-2-yl acetate ((rac)-10a) $\left(\mathrm{CDCl}_{3}, 75 \mathrm{MHz}\right)$.

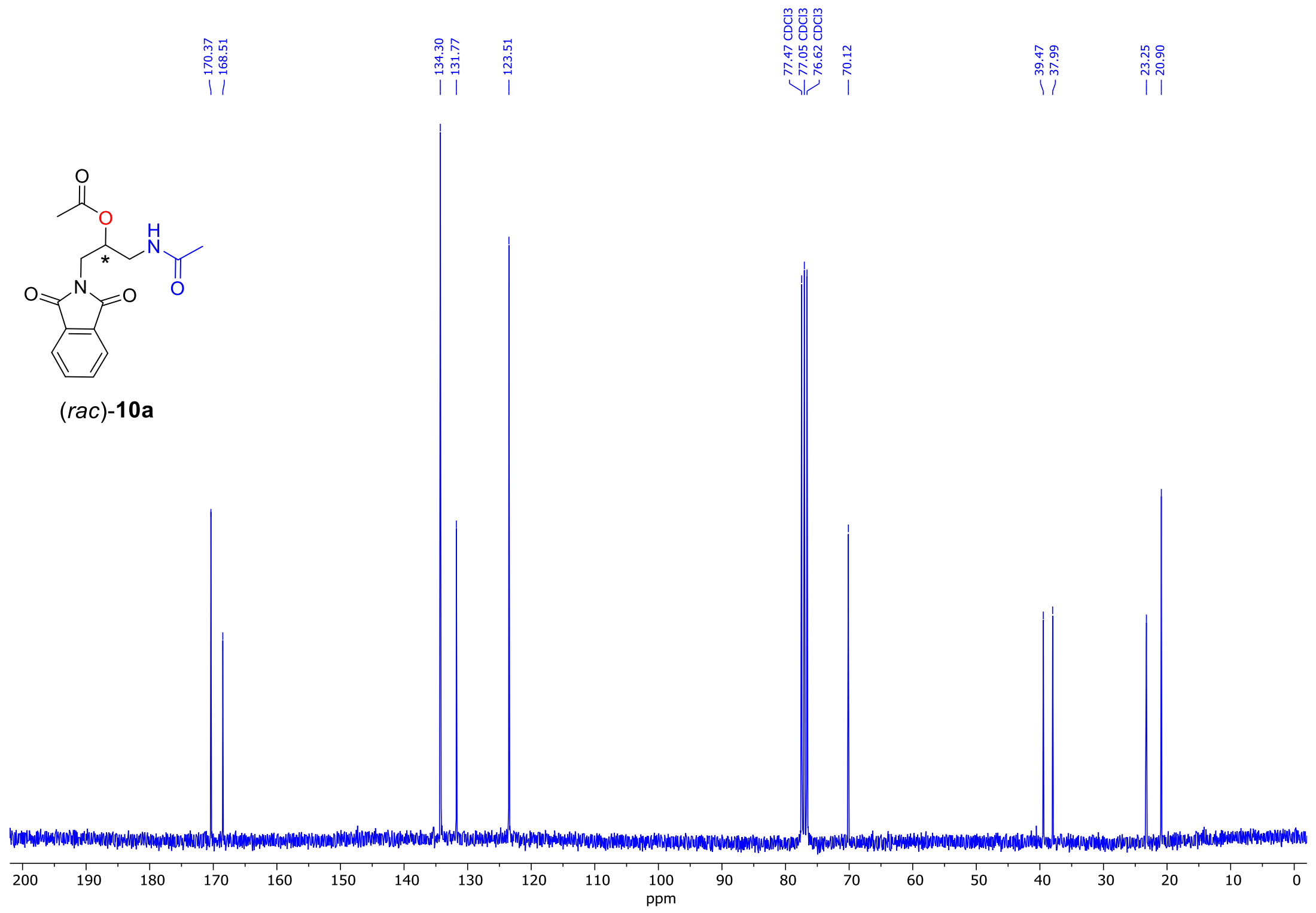


Figure S16: ${ }^{1} \mathrm{H}$ NMR spectrum of 1-acetamido-3-(1,3-dioxoisoindolin-2-yl)propan-2-yl acetate $((R)-10 b)\left(\mathrm{CDCl}_{3}, 300 \mathrm{MHz}\right)$.

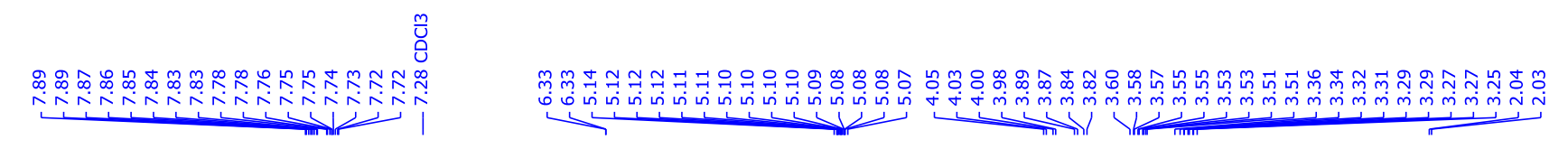

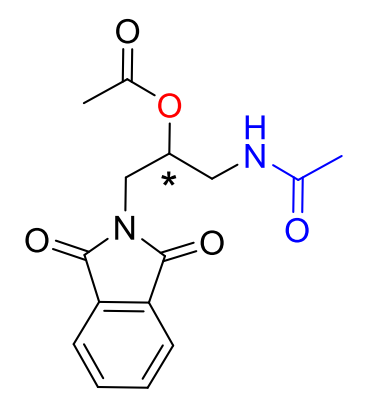

$(R)-10 \mathrm{~b}$

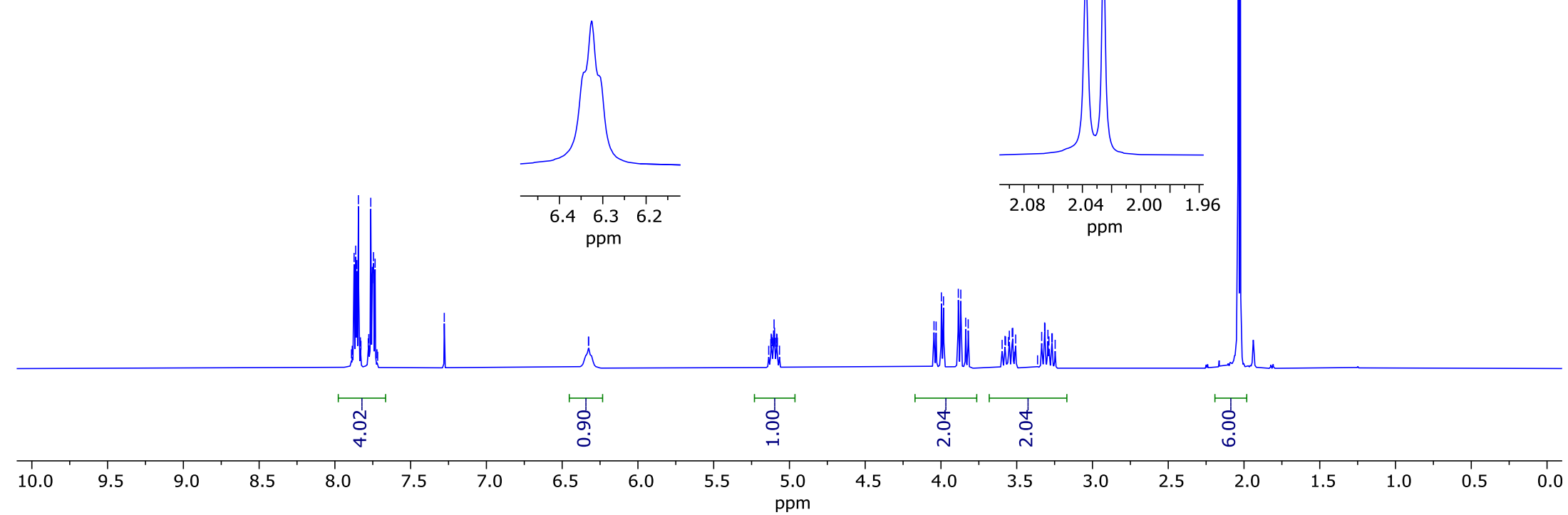


Figure S17. ${ }^{13} \mathrm{C}\left\{{ }^{1} \mathrm{H}\right\}$ NMR spectrum of 1-acetamido-3-(1,3-dioxoisoindolin-2-yl)propan-2-yl acetate $((R)-10 b)\left(\mathrm{CDCl}_{3}, 75 \mathrm{MHz}\right)$.

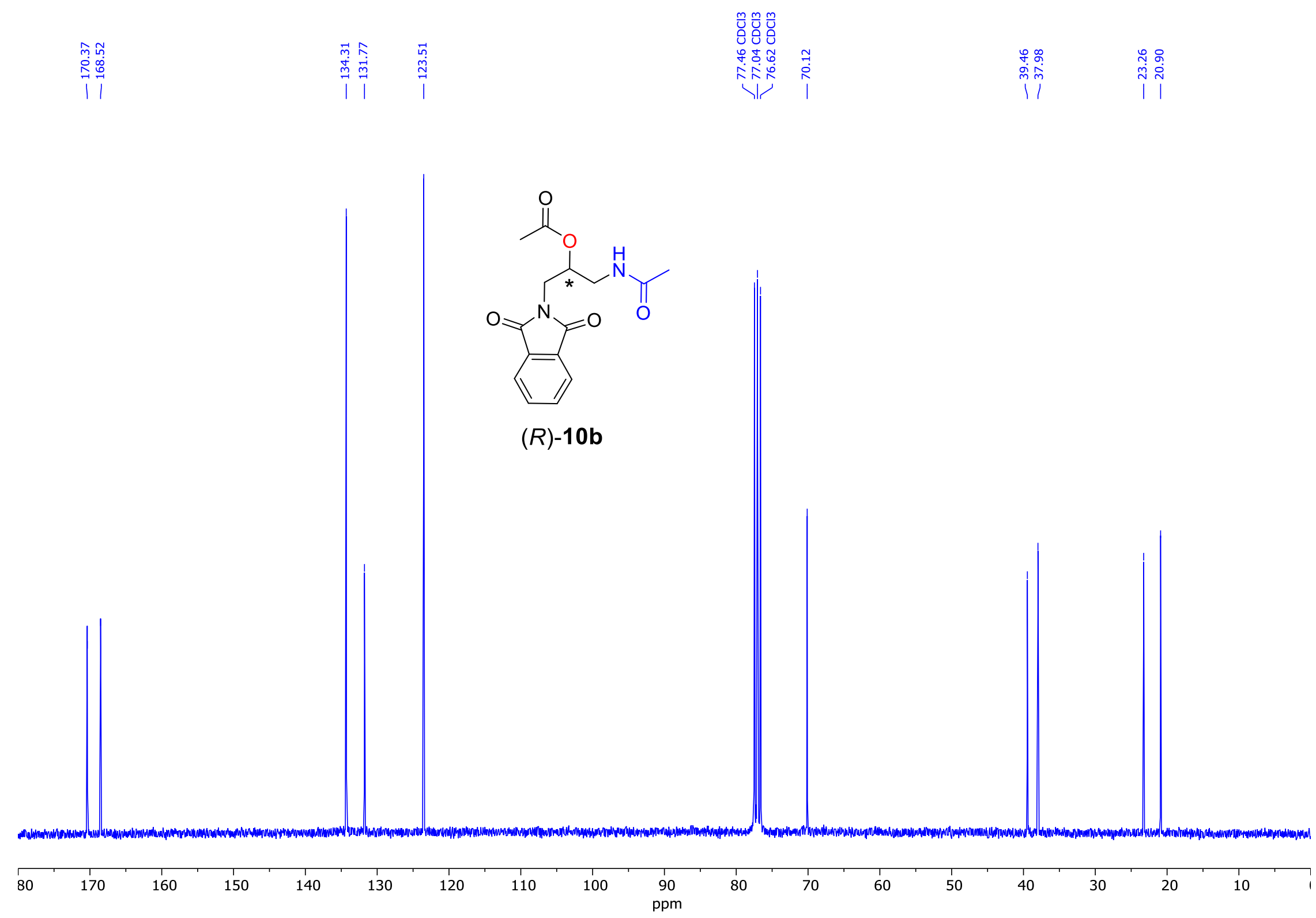


Figure S18: ${ }^{19} \mathrm{~F}$ NMR spectrum of 1-acetamido-3-(1,3-dioxoisoindolin-2-yl)propan-2-yl acetate $\left((R)\right.$-10b) after $\mathrm{KF}$ wash $\left(\mathrm{CDCl}_{3}, 282 \mathrm{MHz}\right)$.

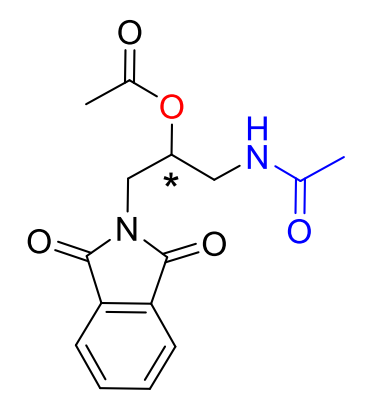

$(R)-10 b$

10

$-30$

$-40$

$-50 \quad-60$

$-70$

$-80 \quad-90$

$-100$

$\begin{array}{llll}-110 & -120 & -130 & -140\end{array}$

$\begin{array}{lllllll}-150 & -160 & -170 & -180 & -190 & -200 & -210\end{array}$ 
Figure S19: ${ }^{1} \mathrm{H}$ NMR spectrum of 1-acetamido-3-phenoxypropan-2-yl acetate ((rac)-12) $\left(\mathrm{CDCl}_{3}, 300 \mathrm{MHz}\right)$.

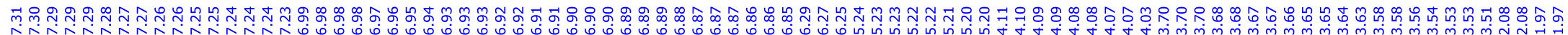

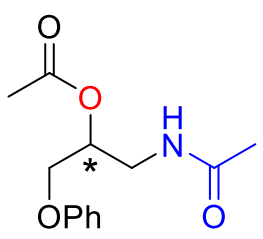

(rac)-12
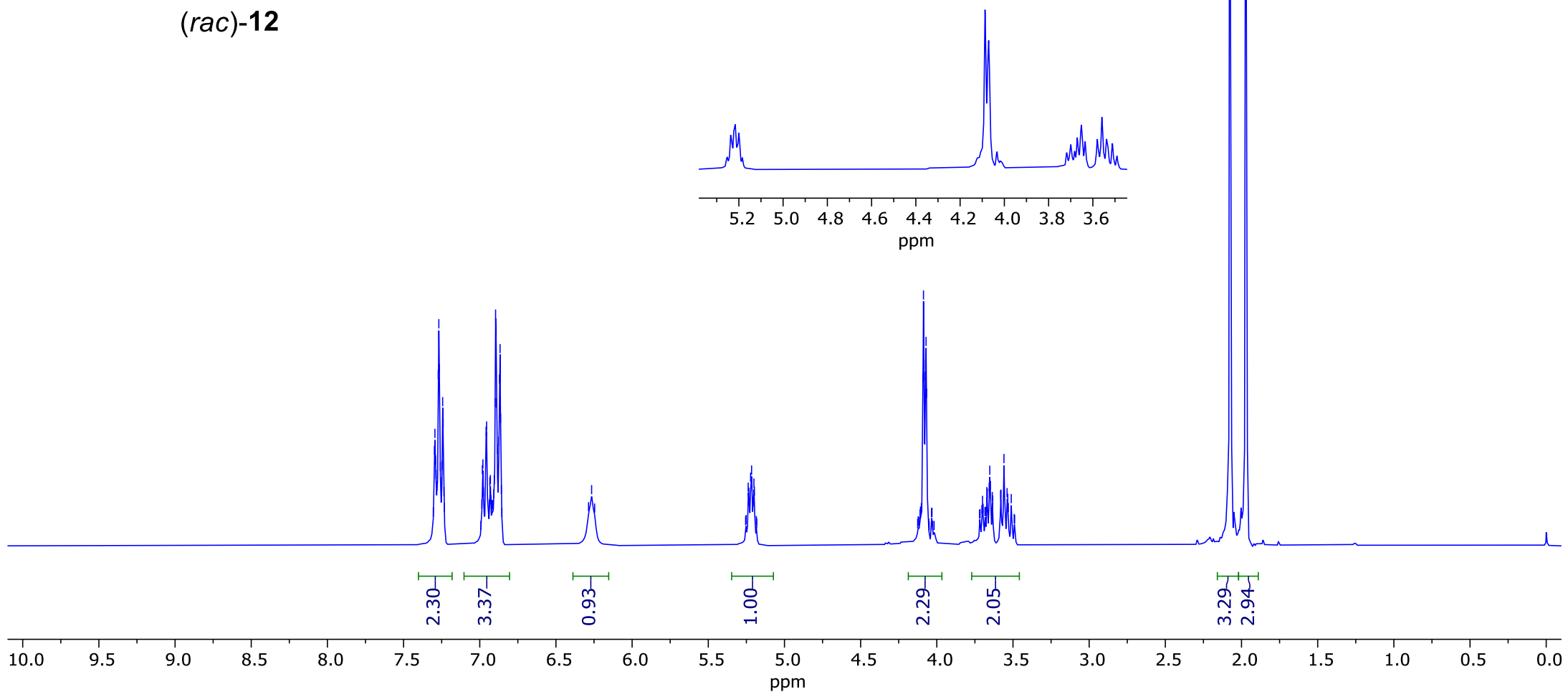
Figure S20: ${ }^{13} \mathrm{C}\left\{{ }^{1} \mathrm{H}\right\}$ NMR spectrum of 1-acetamido-3-phenoxypropan-2-yl acetate $((\mathrm{rac})-\mathbf{1 2})\left(\mathrm{CDCl}_{3}, 75 \mathrm{MHz}\right)$.

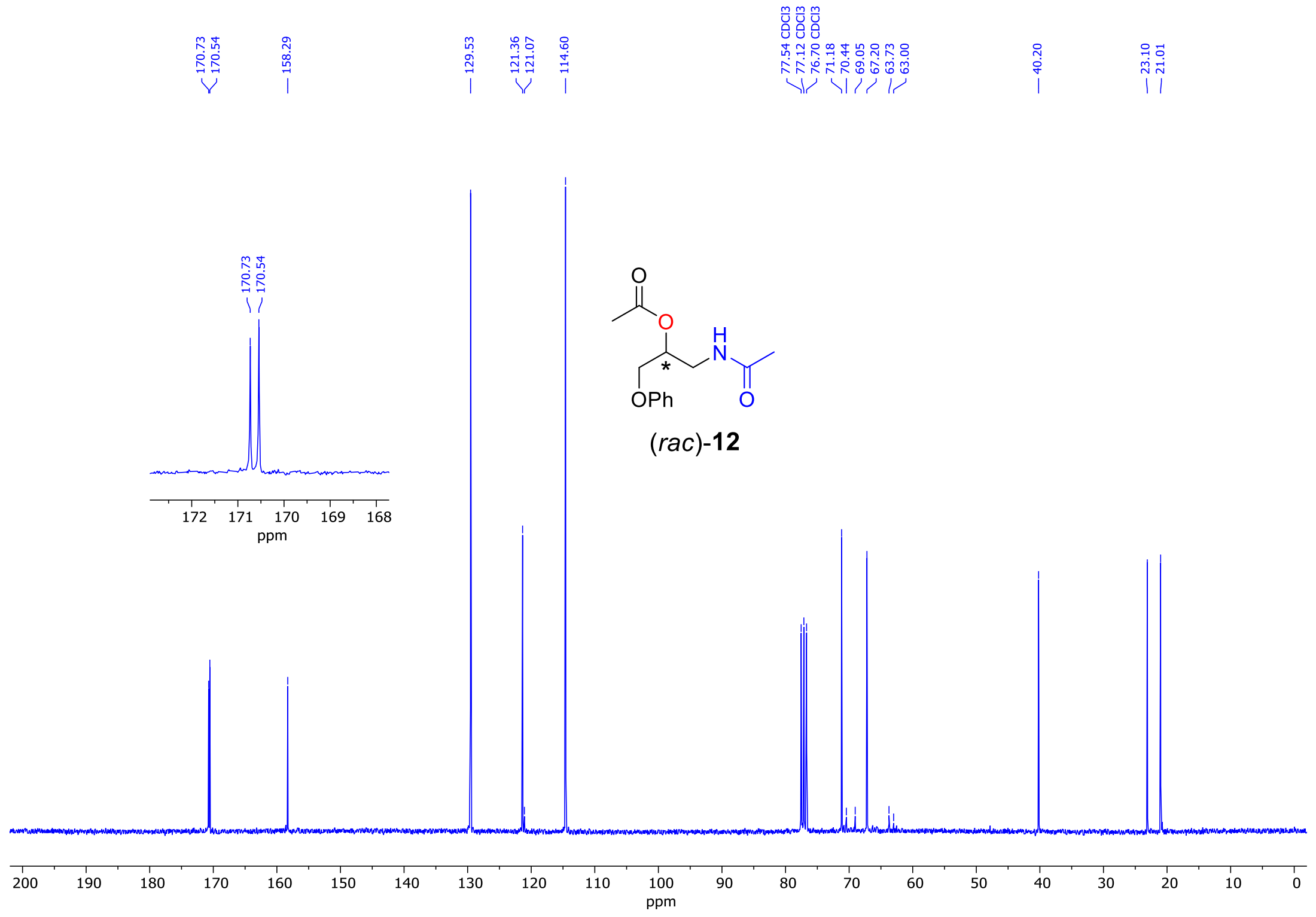


Figure S21: ${ }^{1} \mathrm{H}$ NMR spectrum of 1-acetamido-3-phenoxypropan-2-yl pivalate ((rac)-14) $\left(\mathrm{CDCl}_{3}, 300 \mathrm{MHz}\right)$.

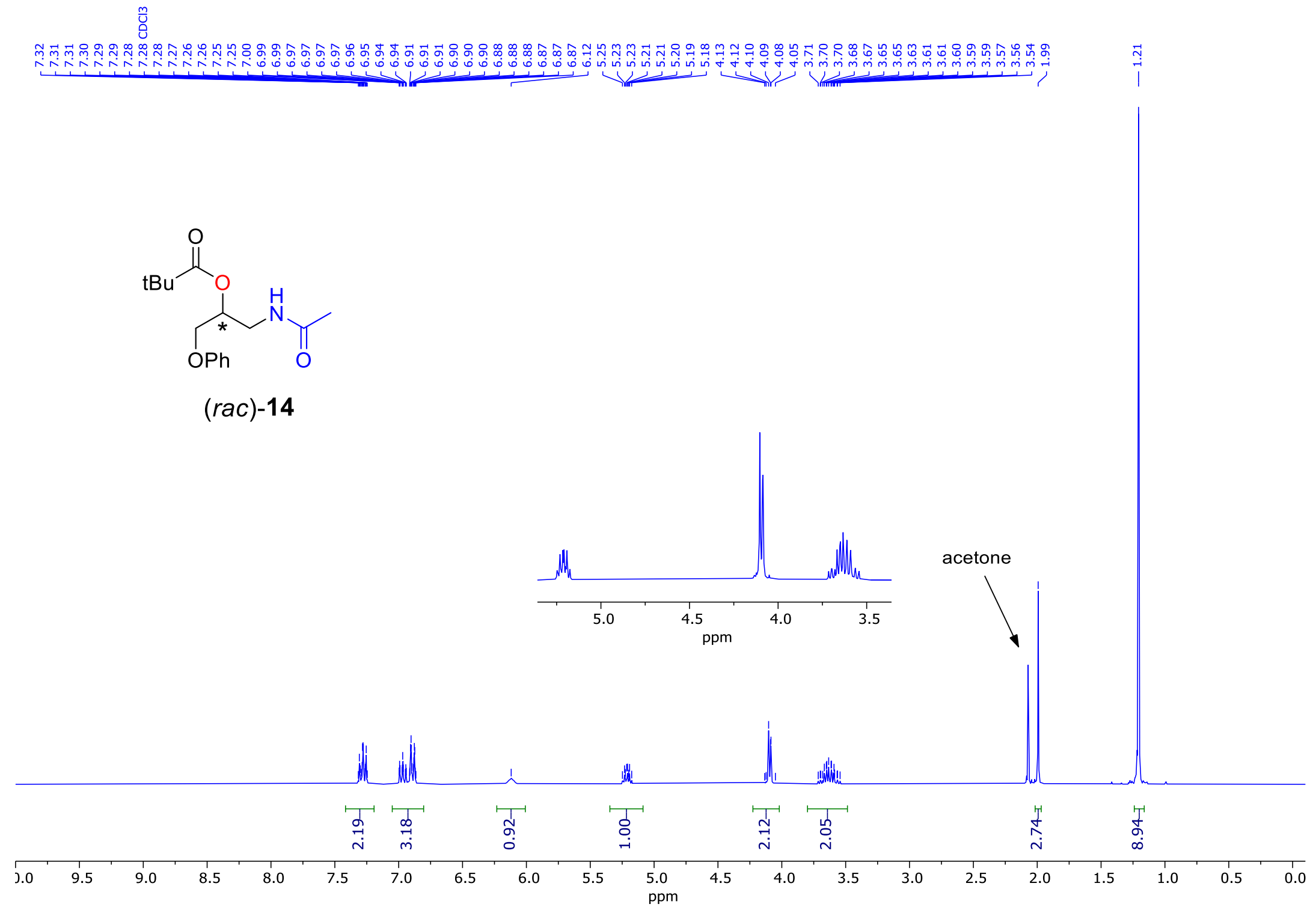


Figure S22: ${ }^{13} \mathrm{C}\left\{{ }^{1} \mathrm{H}\right\}$ NMR spectrum of 1-acetamido-3-phenoxypropan-2-yl pivalate $((\mathrm{rac})-\mathbf{1 4})\left(\mathrm{CDCl}_{3}, 75 \mathrm{MHz}\right)$.

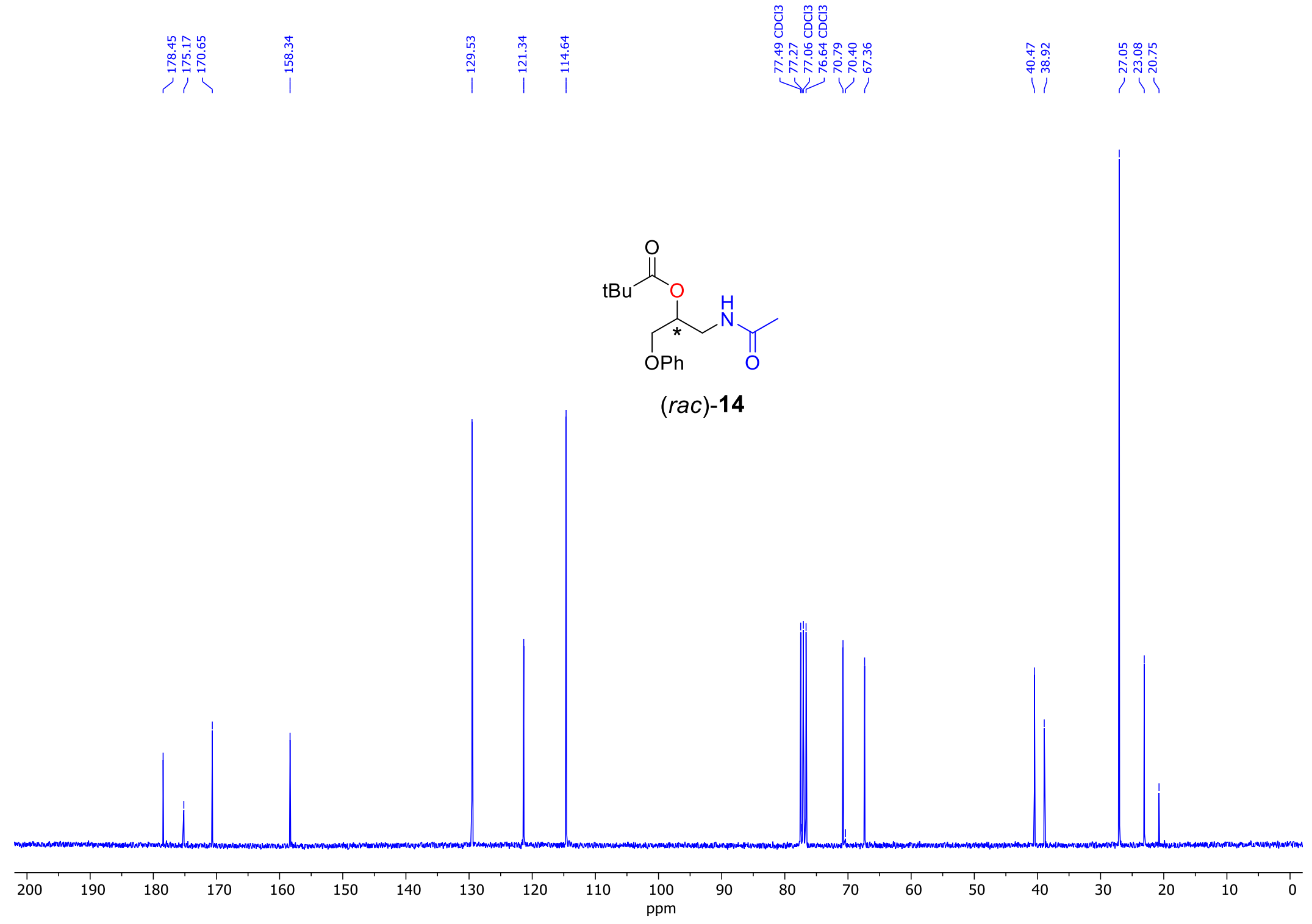


Figure S23: ${ }^{1} \mathrm{H}$ NMR spectrum of 1-acetamido-3-alloxypropan-2-yl acetate ( $\left.(\mathrm{rac})-\mathbf{1 6}\right)\left(\mathrm{CDCl}_{3}, 300 \mathrm{MHz}\right)$.

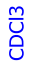

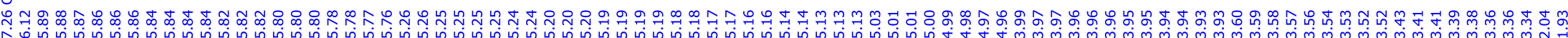<smiles>C=CCOCC(CNC(C)=O)OC(C)=O</smiles>

$($ rac)-16
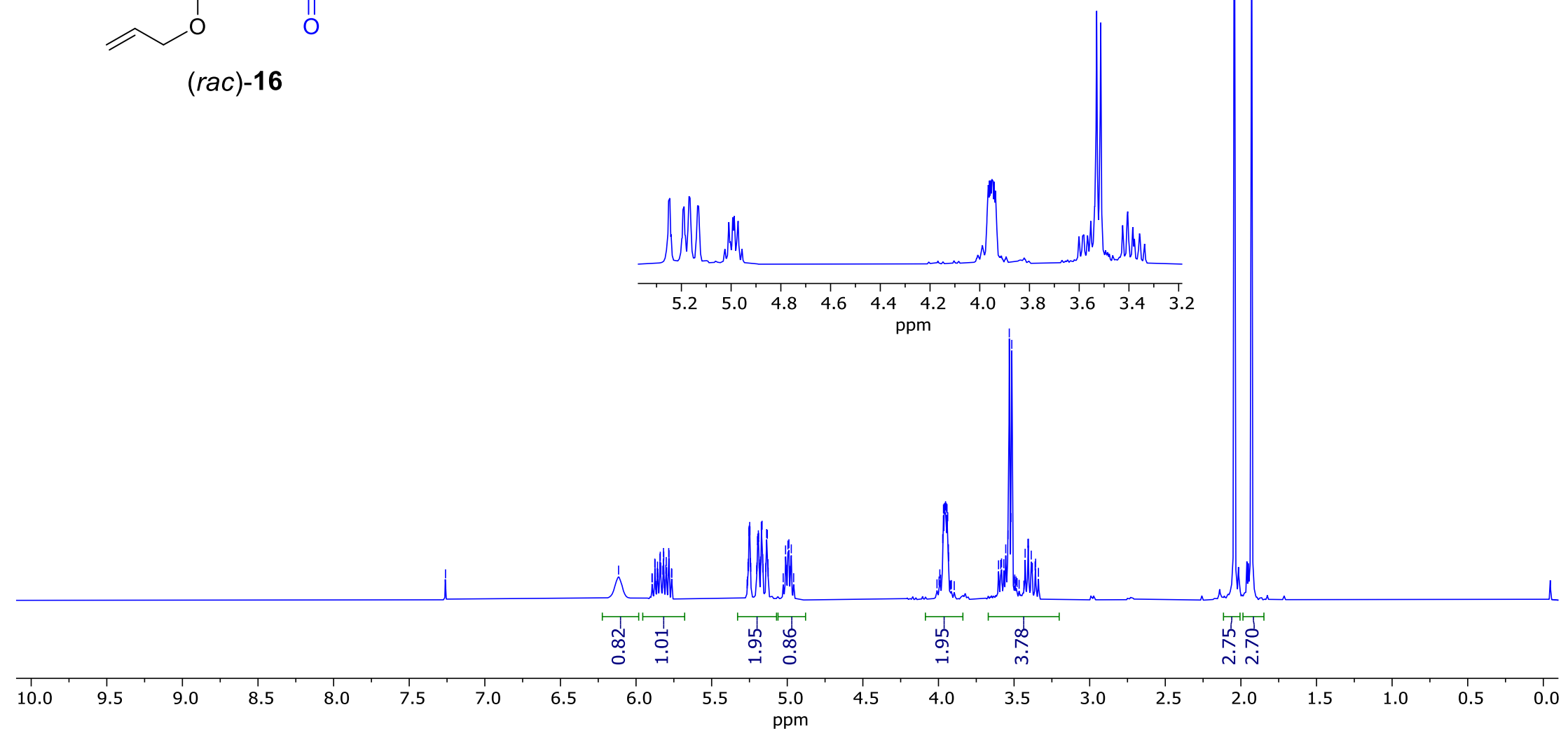
Figure S24: ${ }^{13} \mathrm{C}\left\{{ }^{1} \mathrm{H}\right\}$ NMR spectrum of 1 -acetamido-3-alloxypropan-2-yl acetate $((\mathrm{rac})-\mathbf{1 6})\left(\mathrm{CDCl}_{3}, 75 \mathrm{MHz}\right)$.
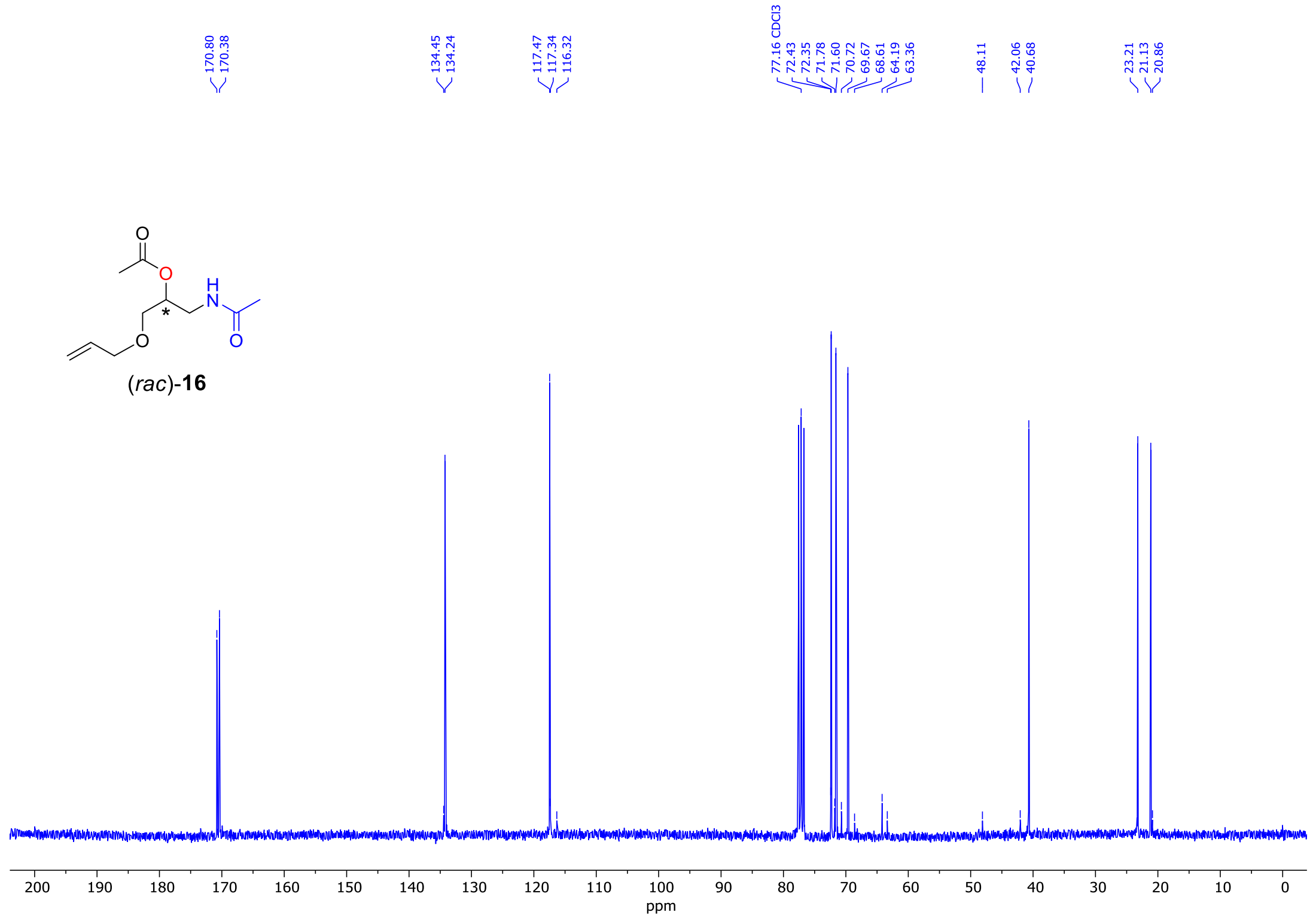
Figure S25: ${ }^{1} \mathrm{H}$ NMR spectrum of 1-acetamido-3-((2-ethylhexyl)oxy)propan-2-yl acetate ((rac)-18) $\left(\mathrm{CDCl}_{3}, 300 \mathrm{MHz}\right)$.
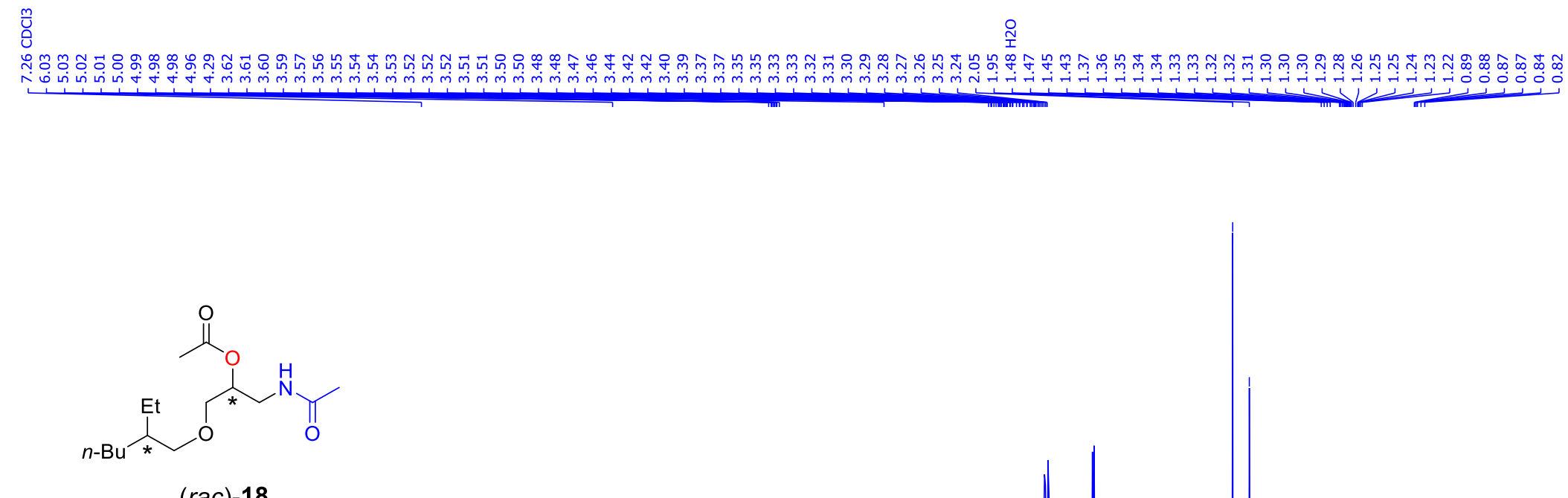

(rac)-18

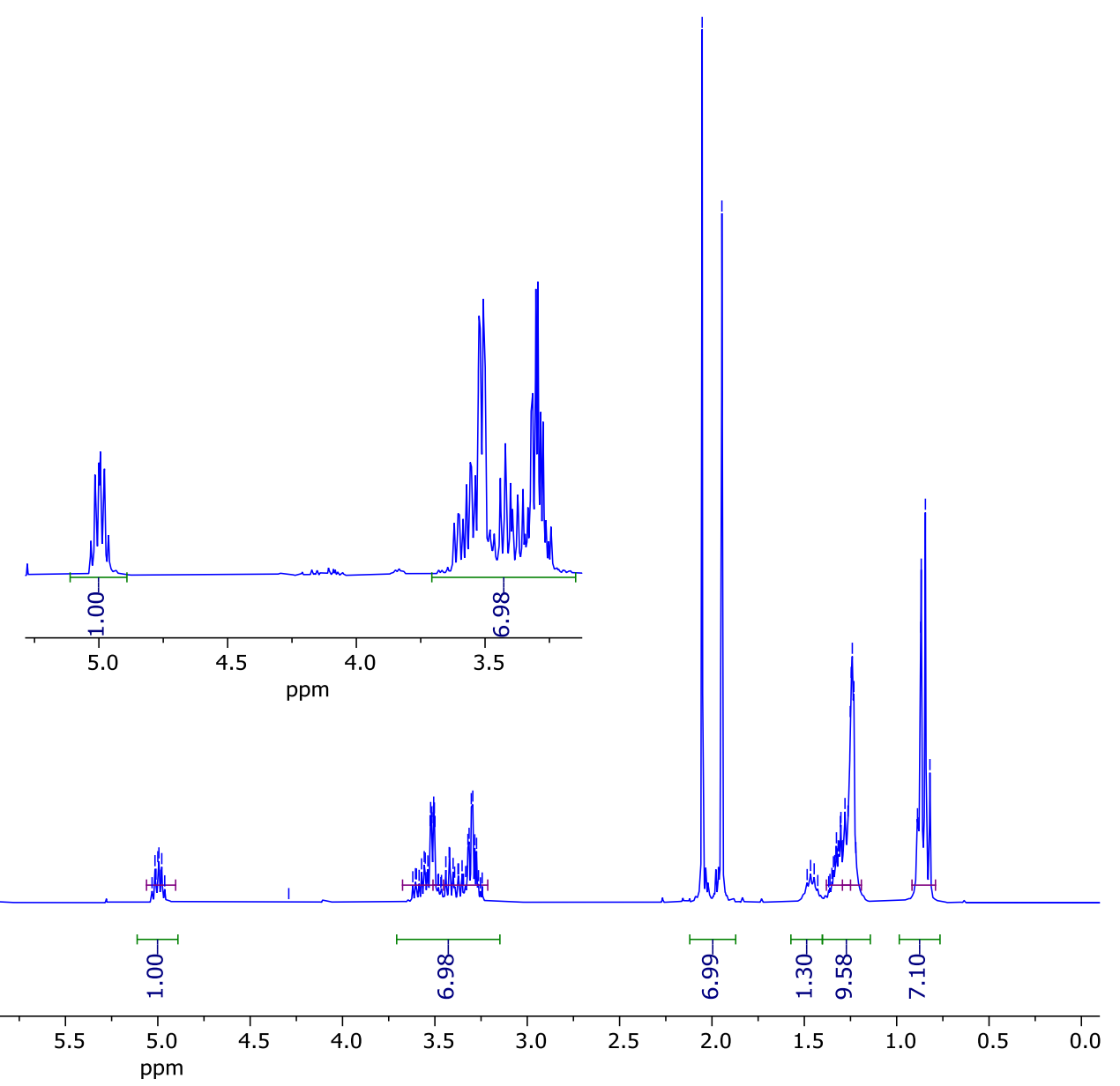


Figure S26: ${ }^{13} \mathrm{C}\left\{{ }^{1} \mathrm{H}\right\}$ NMR spectrum of 1-acetamido-3-((2-ethylhexyl)oxy)propan-2-yl acetate ((rac)-18) $\left(\mathrm{CDCl}_{3}\right.$, $\left.75 \mathrm{MHz}\right)$. Inset: The only set of (partially) resolved peaks for one of the methyl groups indicating $\sim 1: 1$ diastereomeric mixture of products.
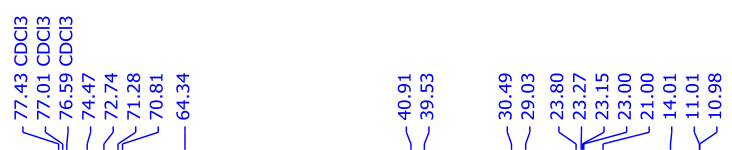

111P<smiles>CC(=O)COC(C)COC(C)=O</smiles>

(rac)-18

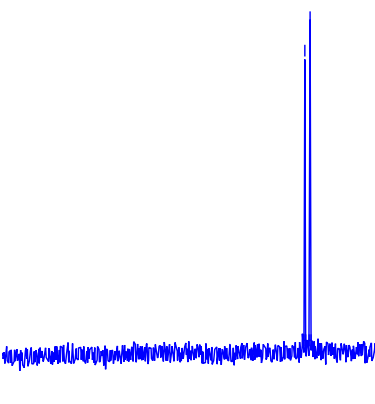


Figure S27: ${ }^{1} \mathrm{H}$ NMR spectrum of 1-acetamido-3-methoxy-2-yl-benzoate ((rac)-19) $\left(\mathrm{CDCl}_{3}, 500 \mathrm{MHz}\right)$.

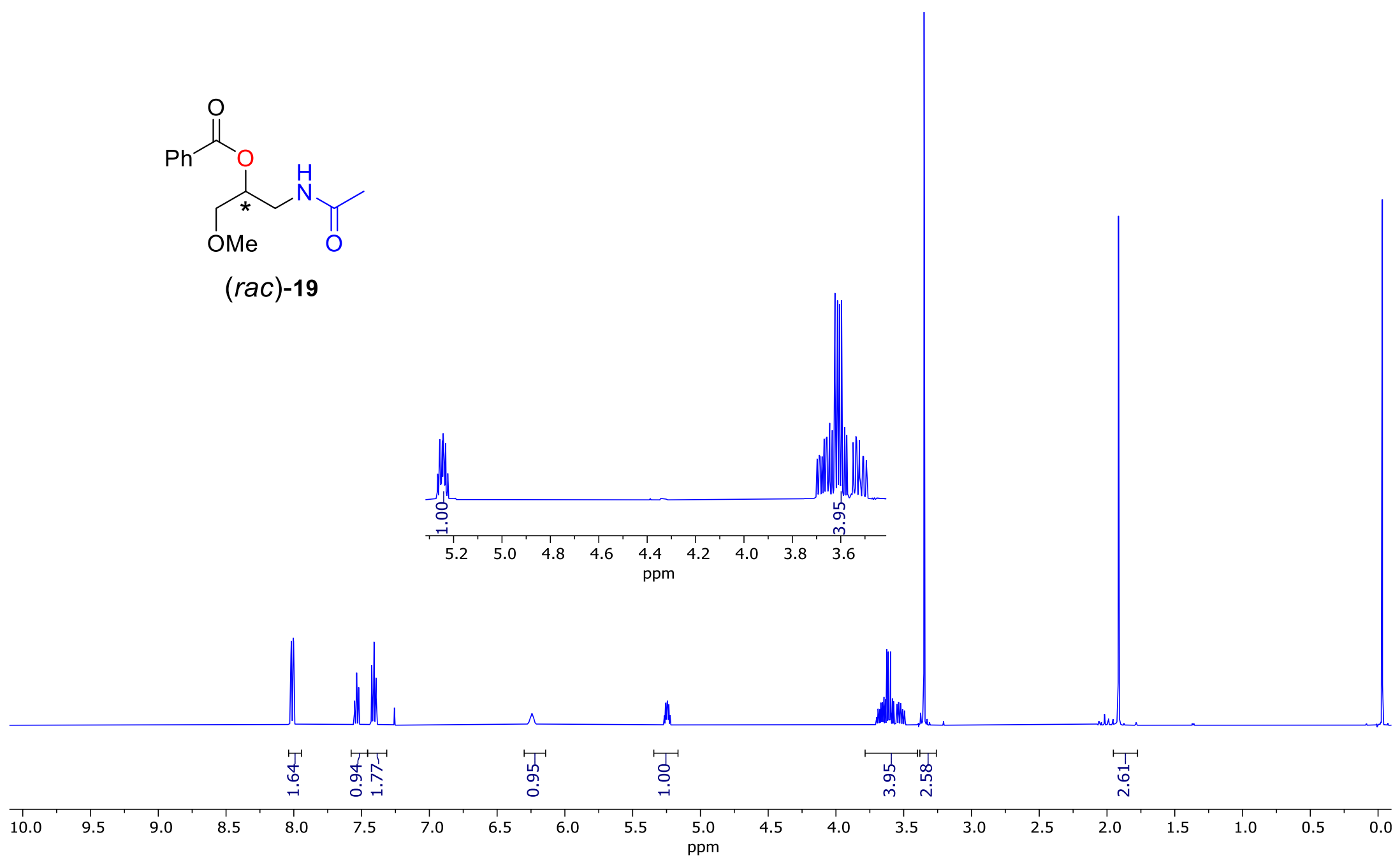


Figure S28. ${ }^{13} \mathrm{C}\left\{{ }^{1} \mathrm{H}\right\}$ NMR spectrum 1-acetamido-3-methoxy-2-yl-benzoate ((rac)-19) $\left(\mathrm{CDCl}_{3}, 126 \mathrm{MHz}\right)$.

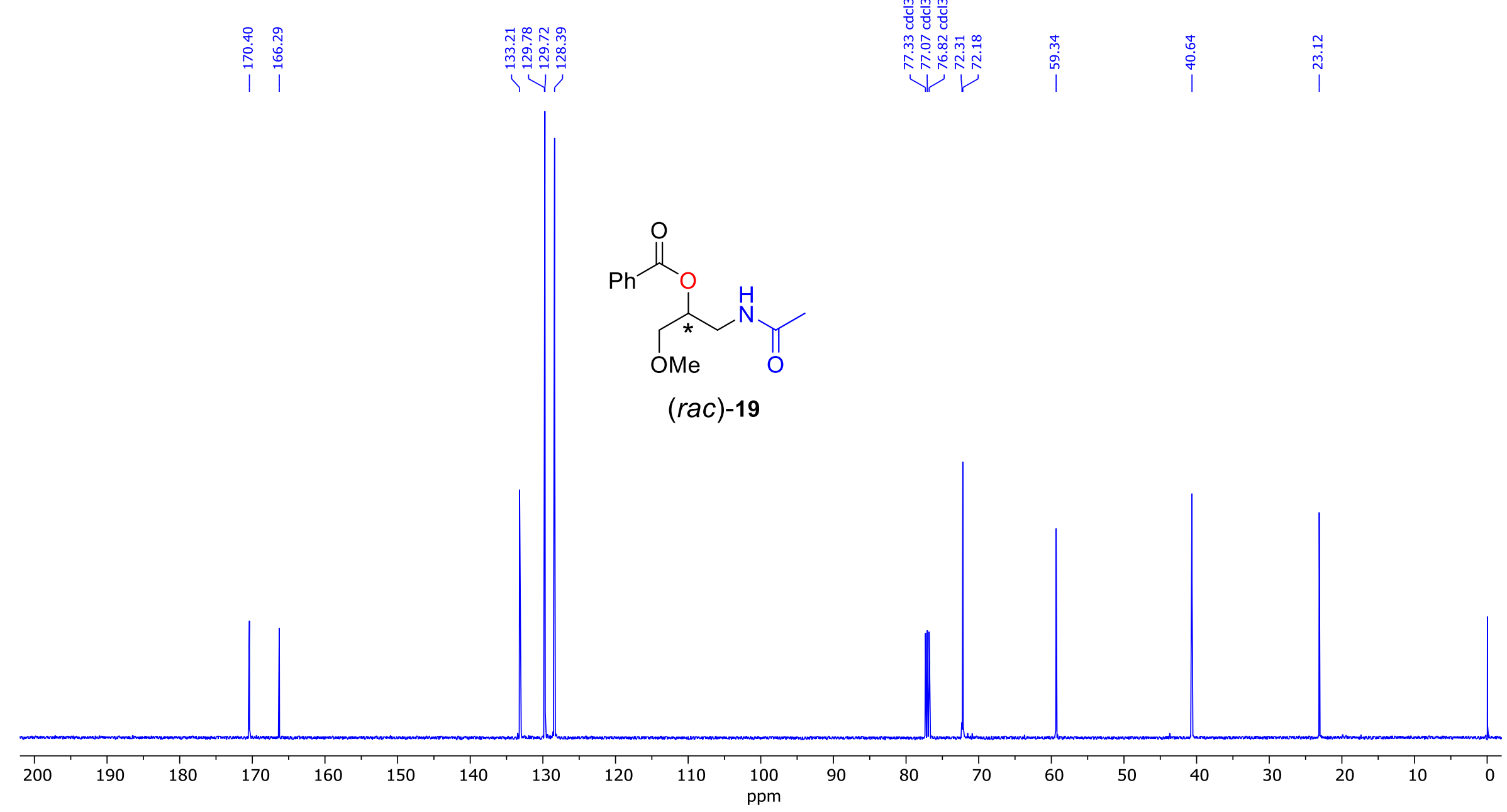


Figure S29: gCOSY NMR spectrum of 1-acetamido-3-methoxy-2-yl-benzoate (( $\mathrm{rac})-19)\left(\mathrm{CDCl}_{3}, 500 \mathrm{MHz}, 500 \mathrm{MHz}\right)$.

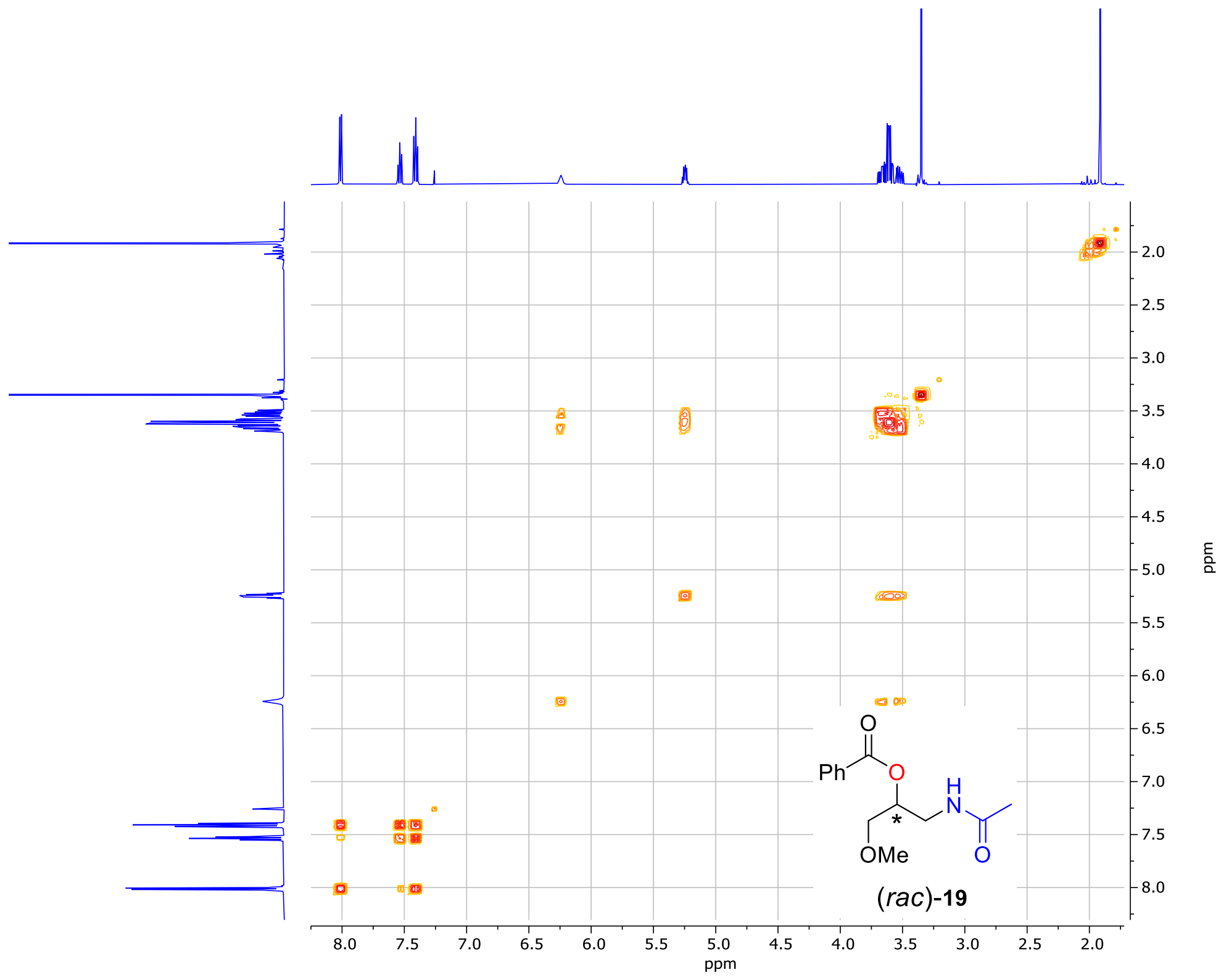


Figure S30: HSQC NMR spectrum of 1-acetamido-3-methoxy-2-yl-benzoate ((rac)-19) $\left(\left(\mathrm{CDCl}_{3}, 500 \mathrm{MHz}, 126 \mathrm{MHz}\right)\right.$.

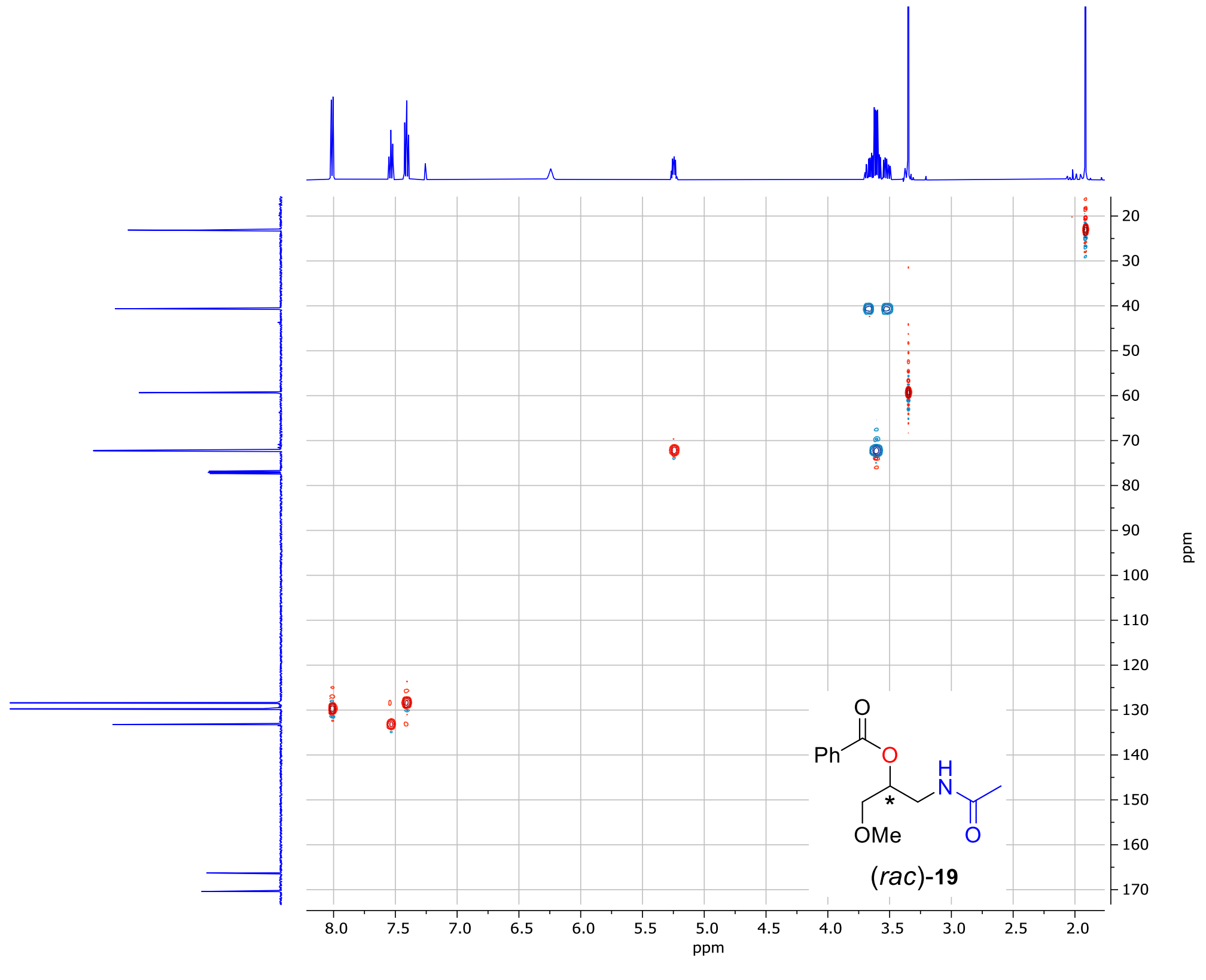


Figure S31: ${ }^{13} \mathrm{C}$ NMR spectrum of crude trans-cyclohexane-1,2-diyl diacetate ((rac)-20) $\left(\mathrm{CDCl}_{3}, 75 \mathrm{MHz}\right)$.

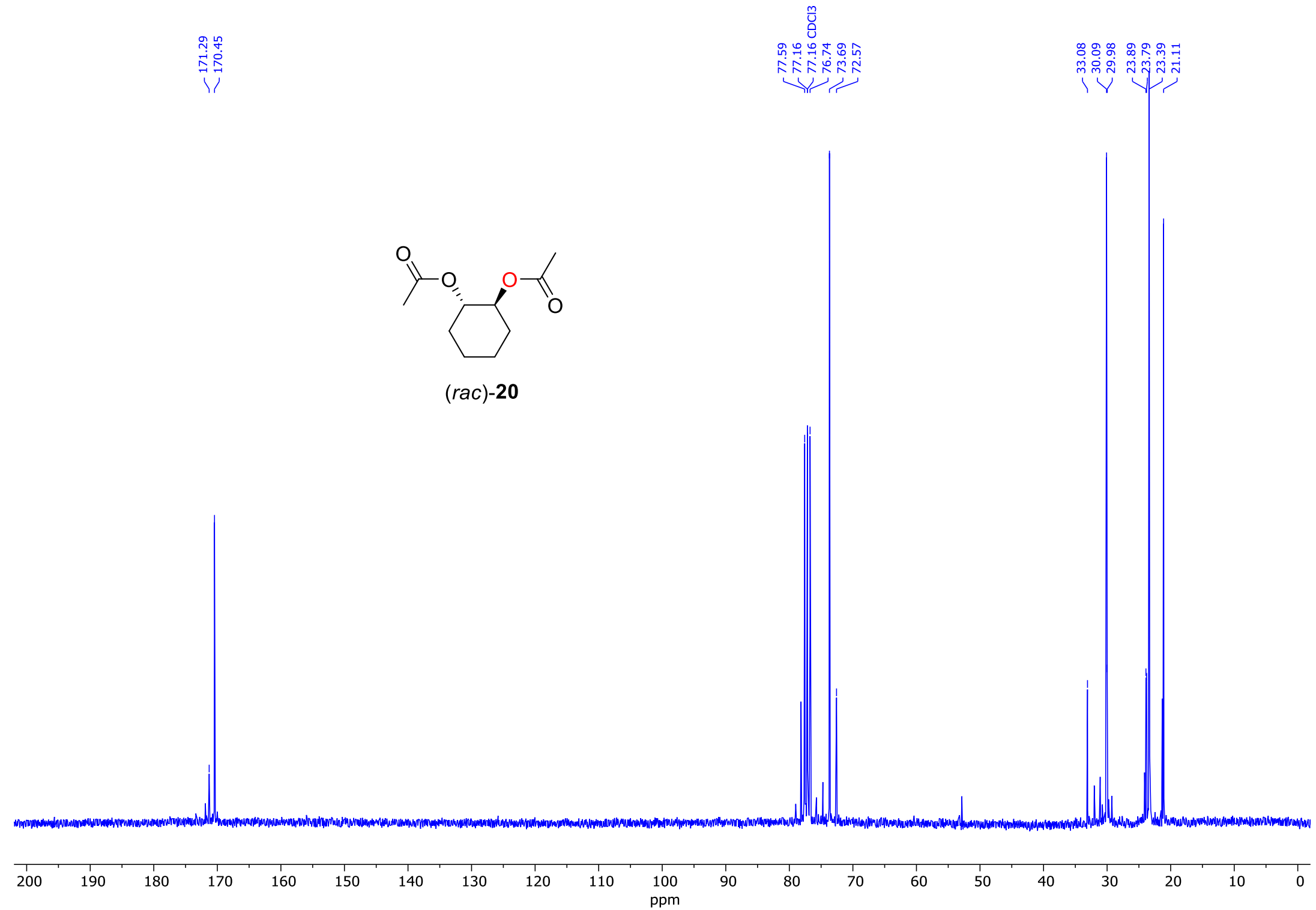


Figure S32: ${ }^{1} \mathrm{H}$ NMR spectrum of purified acetamidohexane-2-yl benzoate ((rac)-21) and 2-acetamidohexane-1-yl benzoate ((rac)-22). Crude $(($ rac $)-21):((r a c)-22)=2.9: 1)\left(\mathrm{CDCl}_{3}, 400 \mathrm{MHz}\right)$.

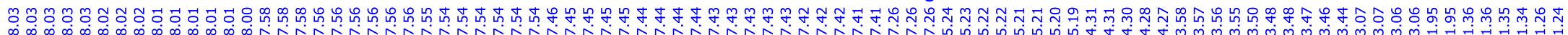<smiles>CC(=O)NC[C@H](C)OC(=O)c1ccccc1</smiles>

(rac)-21<smiles>CC(=O)N[C@@H](C)COC(=O)c1ccccc1</smiles>

$($ rac)-22

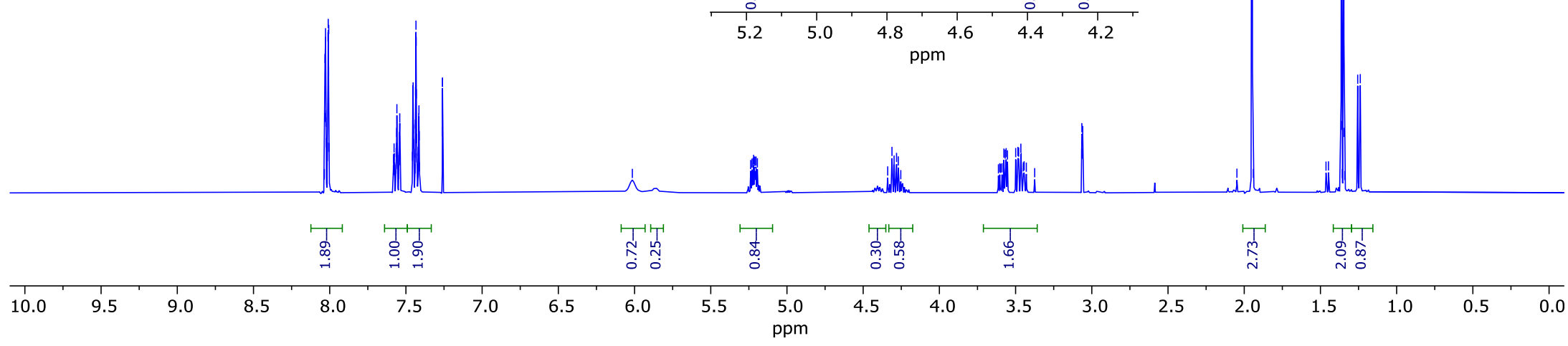


Figure S33: ${ }^{13} \mathrm{C}\left\{{ }^{1} \mathrm{H}\right\}$ NMR of purified 1-acetamidohexane-2-yl benzoate ((rac)-21) and 2-acetamidohexane-1-yl benzoate ((rac)-22). Crude $((r a c)-21):((r a c)-22)=2.9: 1)\left(\mathrm{CDCl}_{3}, 400 \mathrm{MHz}\right)$.

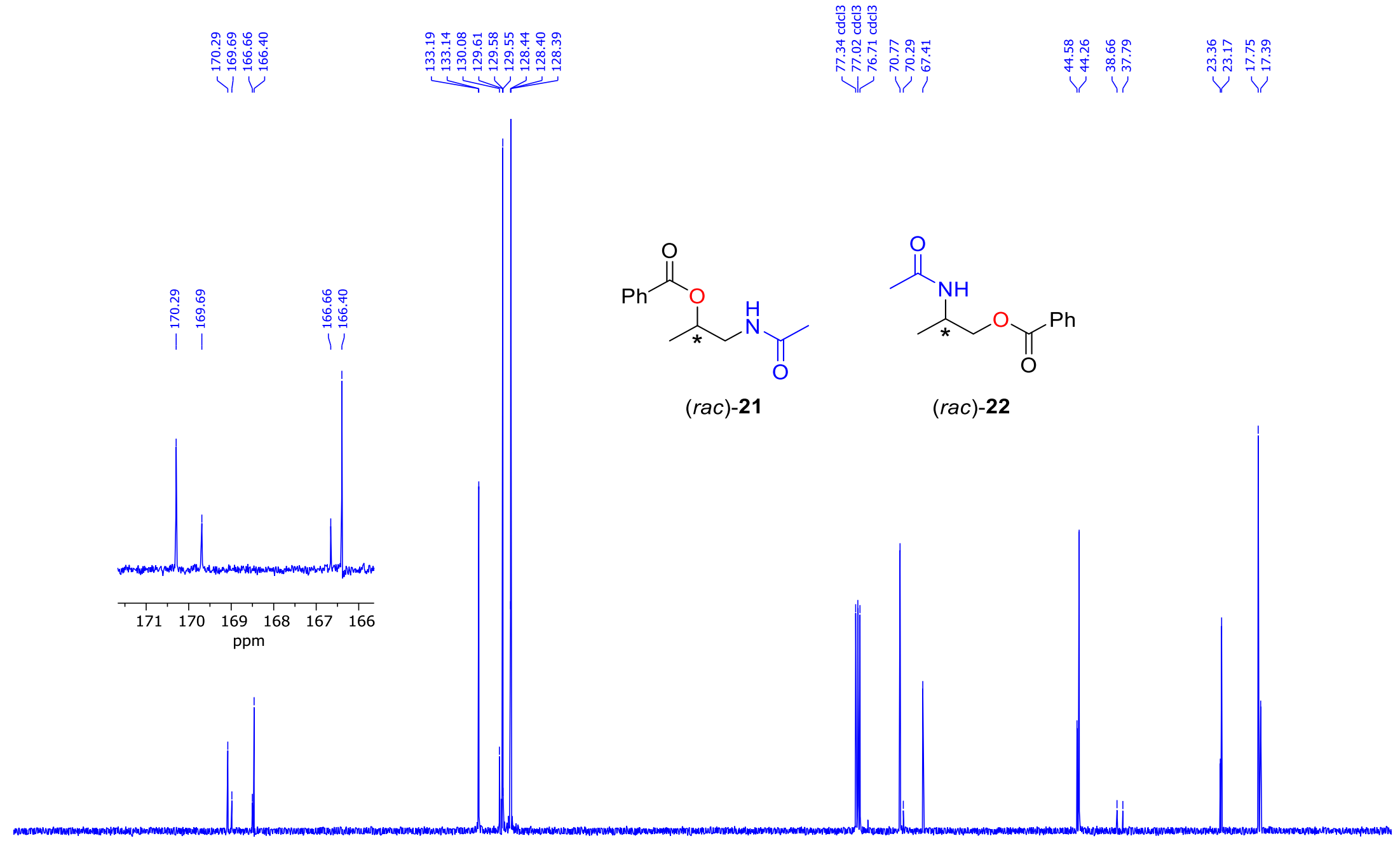

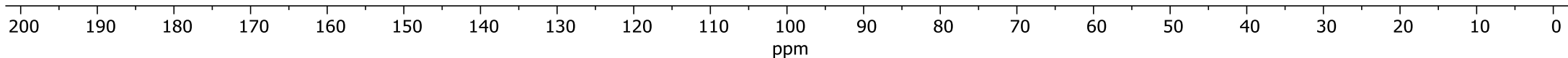


Figure S34. ${ }^{1} \mathrm{H}$ NMR spectrum of 1-acetamidopropan-2-yl acetate ((rac)-23) and 1-acetamidopropan-2-yl acetate ((rac)-24). ((rac)-23):((rac)-24) $=3.8: 1\left(\mathrm{CDCl}_{3}, 300 \mathrm{MHz}\right)$.

峞

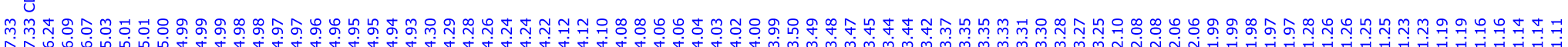

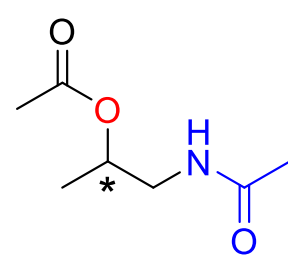

(rac)-23

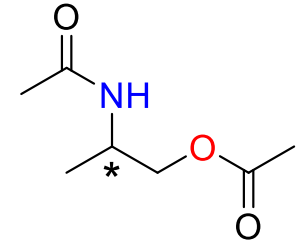

(rac)-24

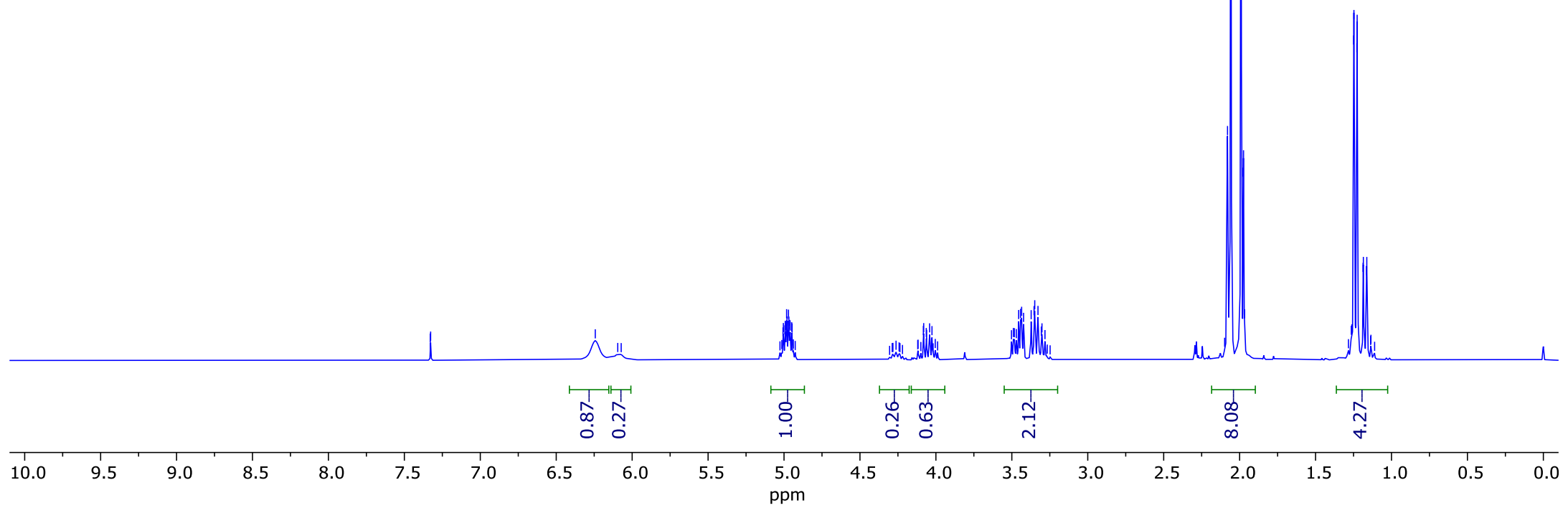


Figure S35: ${ }^{13} \mathrm{C}\left\{{ }^{1} \mathrm{H}\right\}$ NMR spectrum of 1-acetamidopropan-2-yl acetate ((rac)-23) and 2-acetamidopropan-1-yl acetate ((rac)-24). ((rac)-23): $((\mathrm{rac})-24)=3.7: 1\left(\mathrm{CDCl}_{3}, 75 \mathrm{MHz}\right)$.

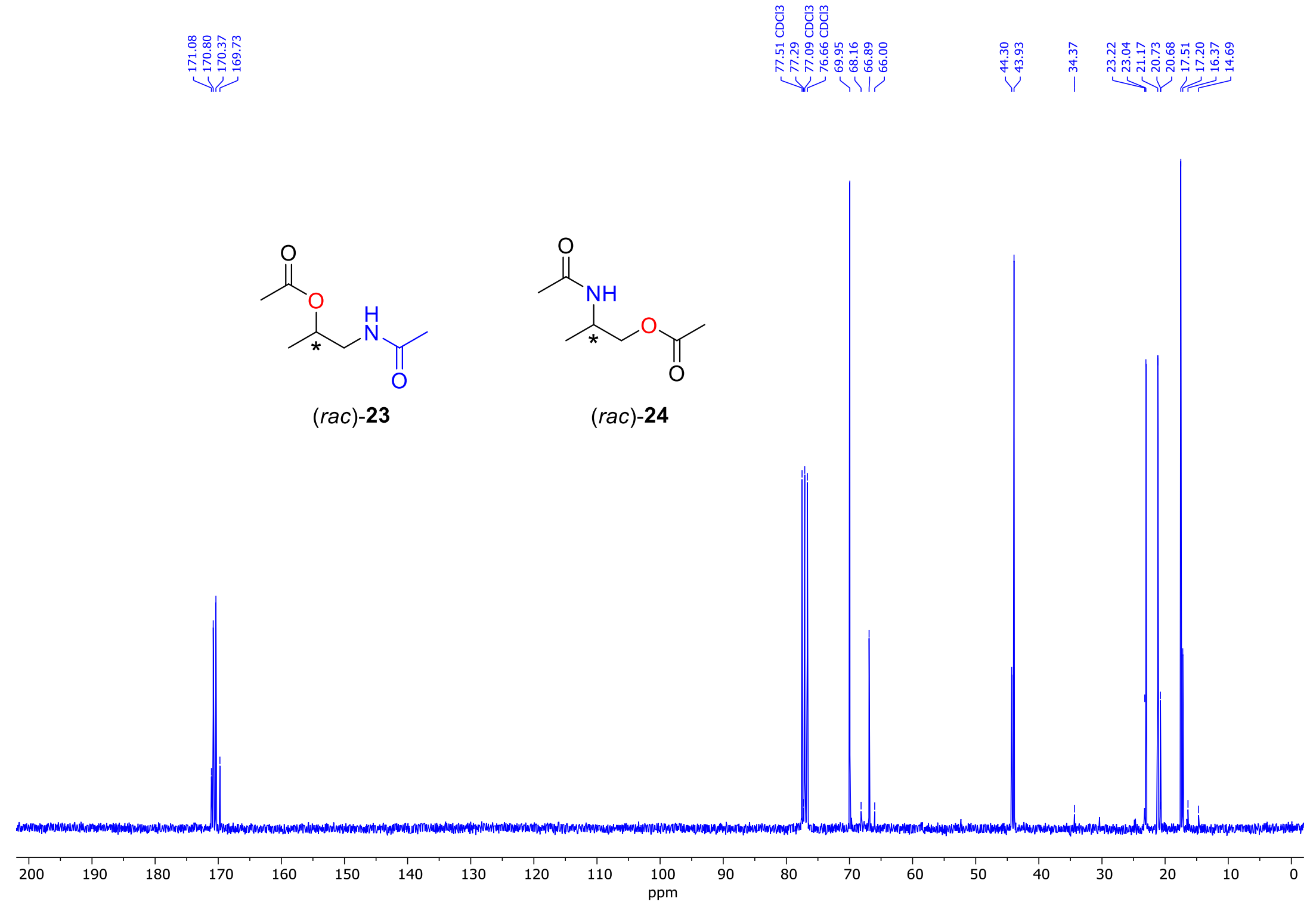


Figure S36: ${ }^{1} \mathrm{H}$ NMR spectrum of 1-benzamidohexane-2-yl benzoate ((rac)-25) and 2-benzamidohexane-1 yl benzoate ((rac)-26). ((rac)-25): $(($ rac $)-26)=2.3: 1\left(\mathrm{CDCl}_{3}, 400 \mathrm{MHz}\right)$

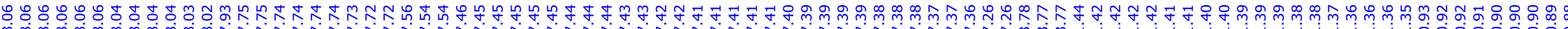

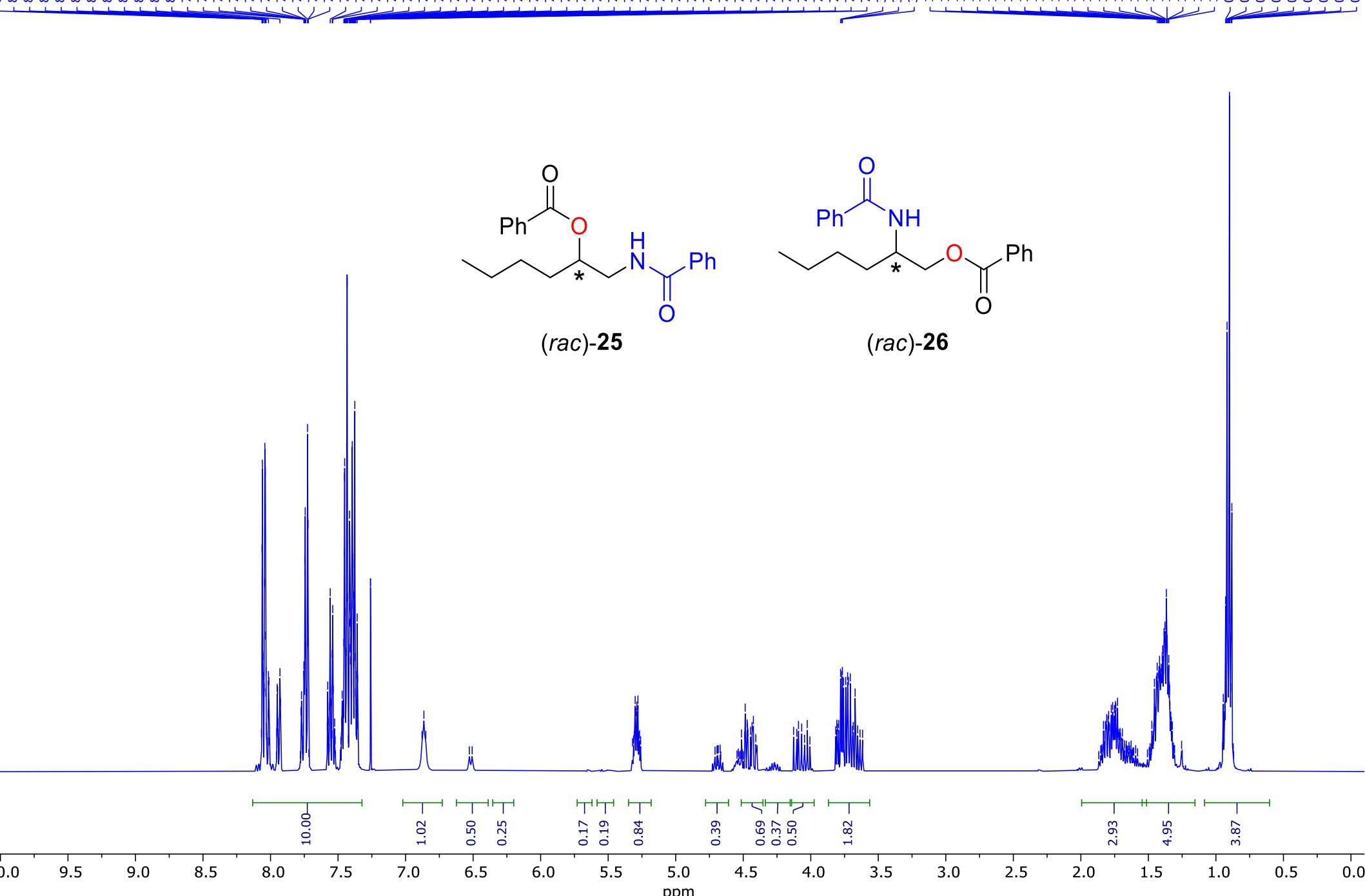


Figure S37: ${ }^{13} \mathrm{C}\left\{{ }^{1} \mathrm{H}\right\}$ NMR spectrum of 1-benzamidohexane-2-yl benzoate ((rac)-25) and 2-benzamidohexane-1-yl benzoate ((rac)-26). ((rac)25): $($ rac $)-26)=2.3: 1\left(\mathrm{CDCl}_{3}, 100 \mathrm{MHz}\right)$.

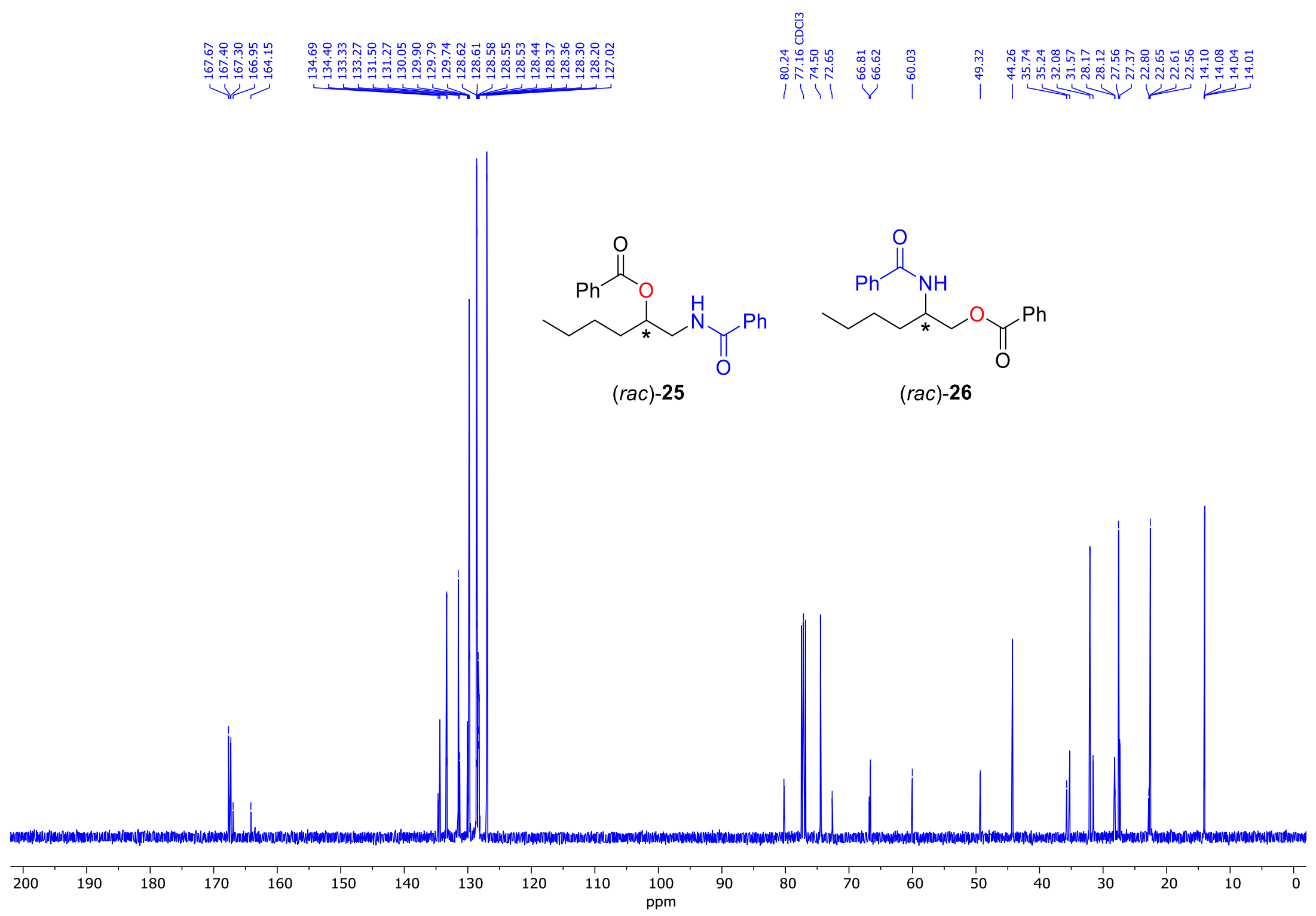


Figure S38: ${ }^{1} \mathrm{H}$ NMR spectrum of purified 1-acetamidohexane-2-yl benzoate ((rac)-27) and 2-acetamidohexane-1-yl benzoate ((rac)-28). Crude $((r a c)-27):((r a c)-28)=2.9: 1)\left(\mathrm{CDCl}_{3}, 400 \mathrm{MHz}\right)$.

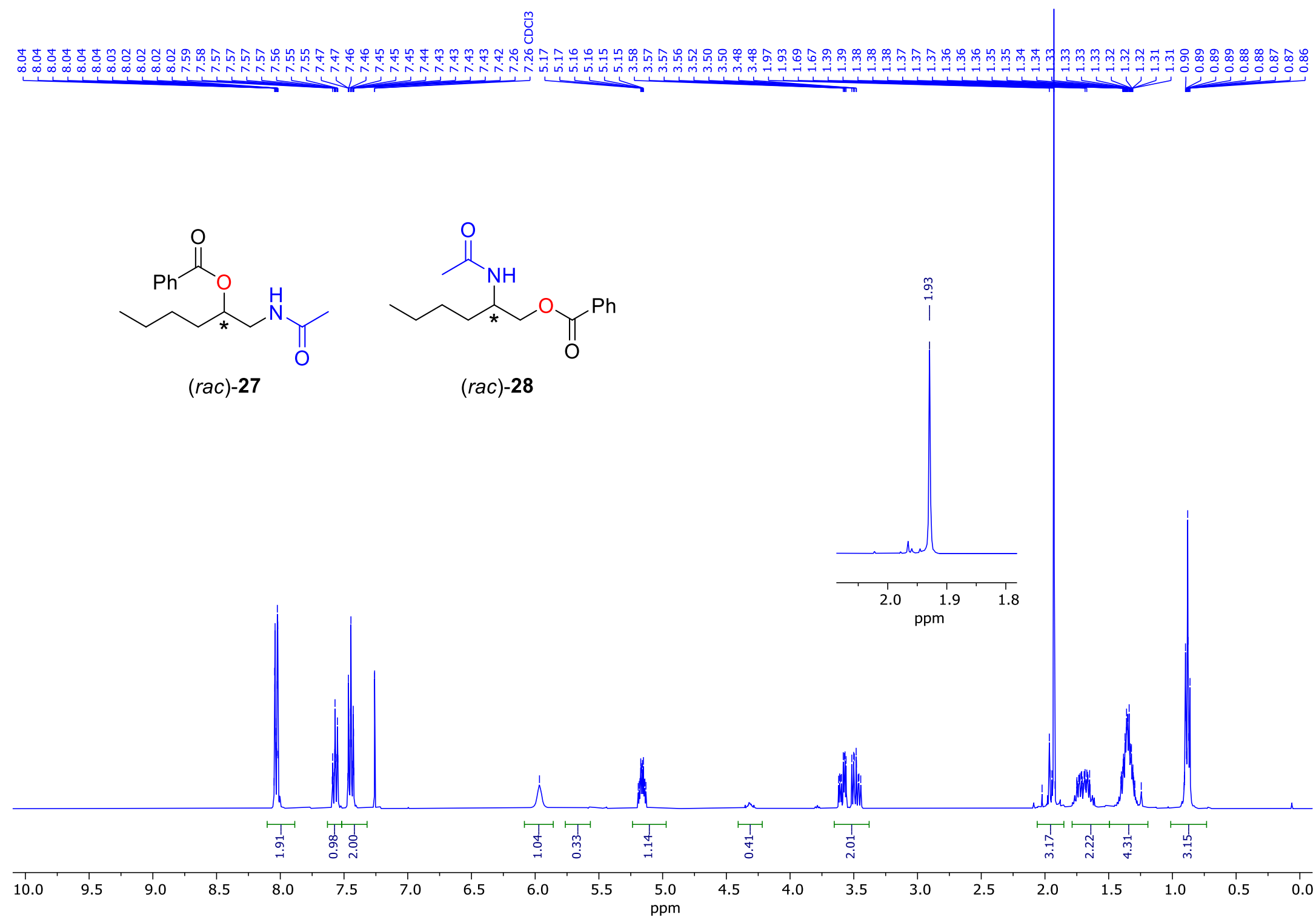


Figure S39: ${ }^{13} \mathrm{C}\left\{{ }^{1} \mathrm{H}\right\}$ NMR of purified 1-acetamidohexane-2-yl benzoate ((rac)-27) and 2-acetamidohexane-1-yl benzoate ((rac)-28). Crude $((r a c)-27):((r a c)-28)=2.9: 1)\left(\mathrm{CDCl}_{3}, 100 \mathrm{MHz}\right)$.

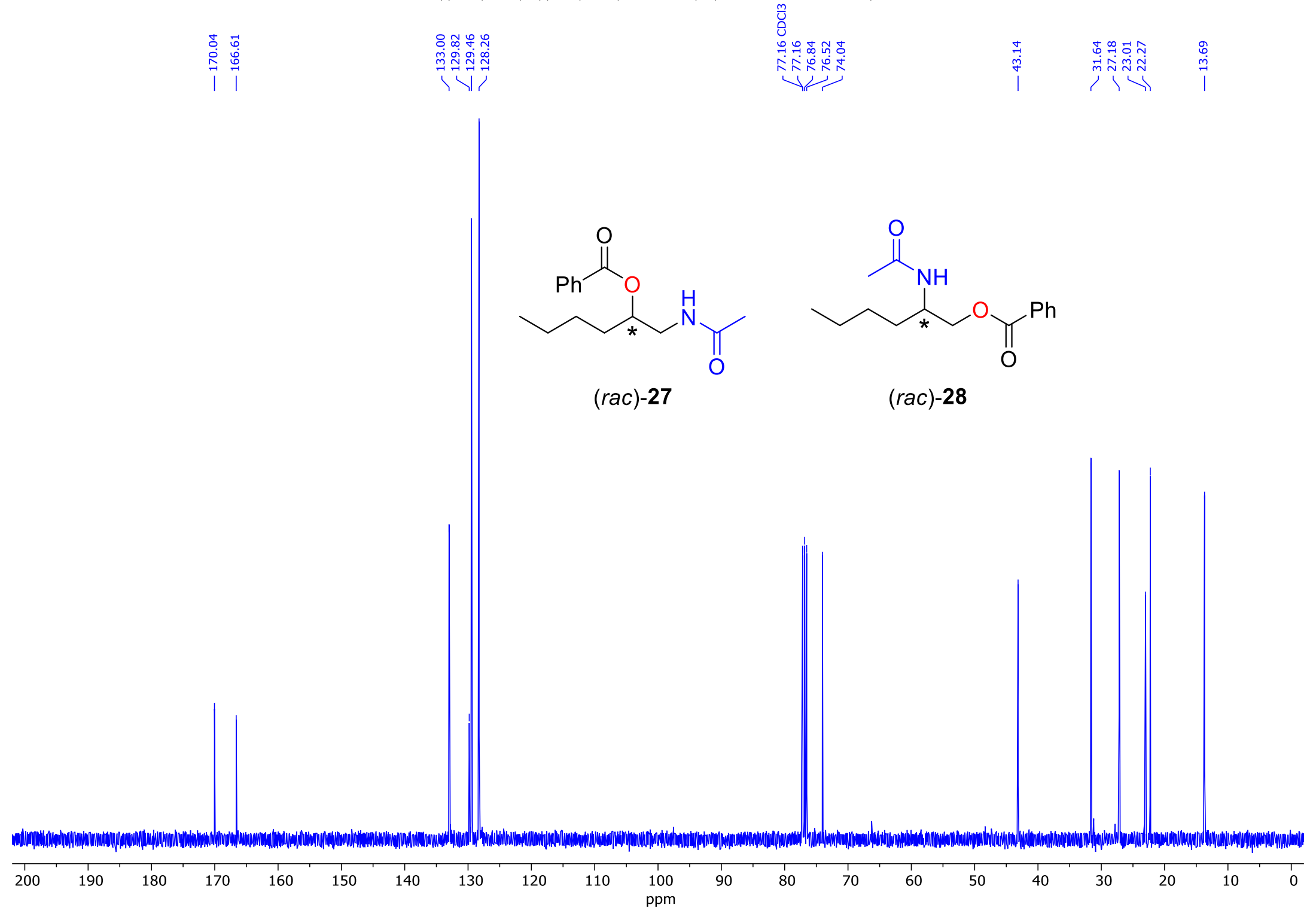


Figure S40: ${ }^{1} \mathrm{H}$ NMR spectrum of 5-butyl-2-methyl-4,5-dihydrooxazole ((rac)-29) and 4-butyl-2-methyl-4,5-dihydrooxazole ((rac)-30). ((rac)29): $((\mathrm{rac})-30)=0.9: 1\left(\mathrm{CDCl}_{3}, 400 \mathrm{MHz}\right)$.

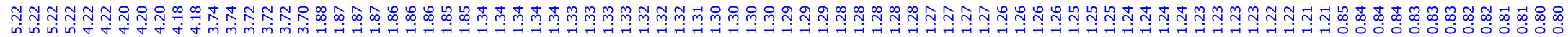

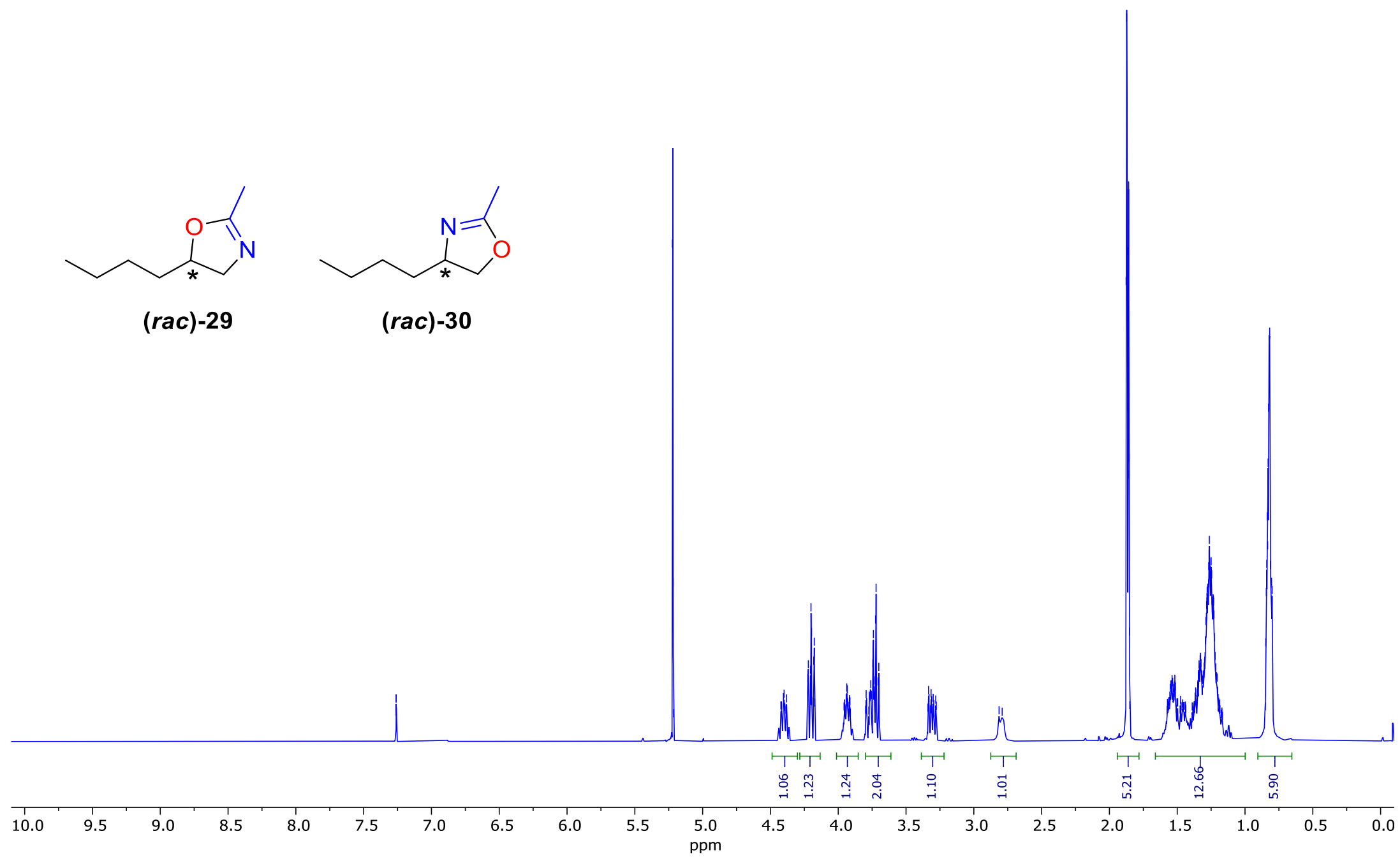


Figure S41: ${ }^{13} \mathrm{C}\left\{{ }^{1} \mathrm{H}\right\}$ NMR spectrum of 5-butyl-2-methyl-4,5-dihydrooxazole ((rac)-29) and 4-butyl-2-methyl-4,5-dihydrooxazole ((rac)-30). $(($ rac $)-29):((r a c)-30)=0.9: 1\left(\mathrm{CDCl}_{3}, 101 \mathrm{MHz}\right)$.

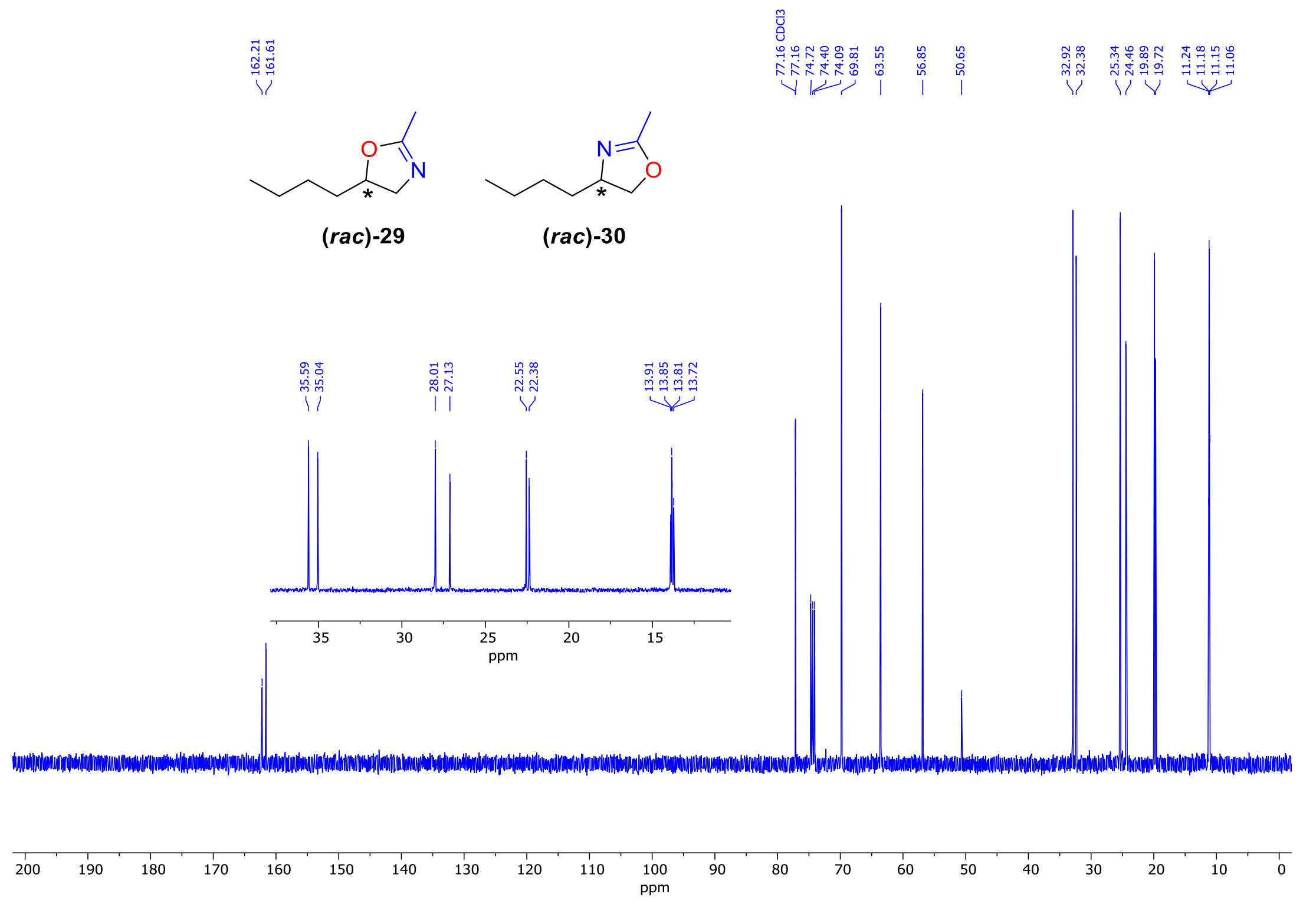




\section{Representative NMR Spectra of diesters, monoesters, and dioxonium cation exchange with esters}

Figure S42: ${ }^{1} \mathrm{H}$ NMR spectrum of 3-chloro-2-hydroxypropyl acetate ((rac)-1a) and 1-chloro-3-hydroxypropan-2-yl acetate ((rac)-1b. ((rac)1a):((rac)-1b = 7.6:1 $\left(\mathrm{CDCl}_{3}, 300 \mathrm{MHz}\right)$.<smiles>CC(=O)OC[C@@H](O)CCl</smiles>

(rac)-1a<smiles>CC(=O)O[C@H](CO)CCl</smiles>

$($ rac) $-\mathbf{1 b}$

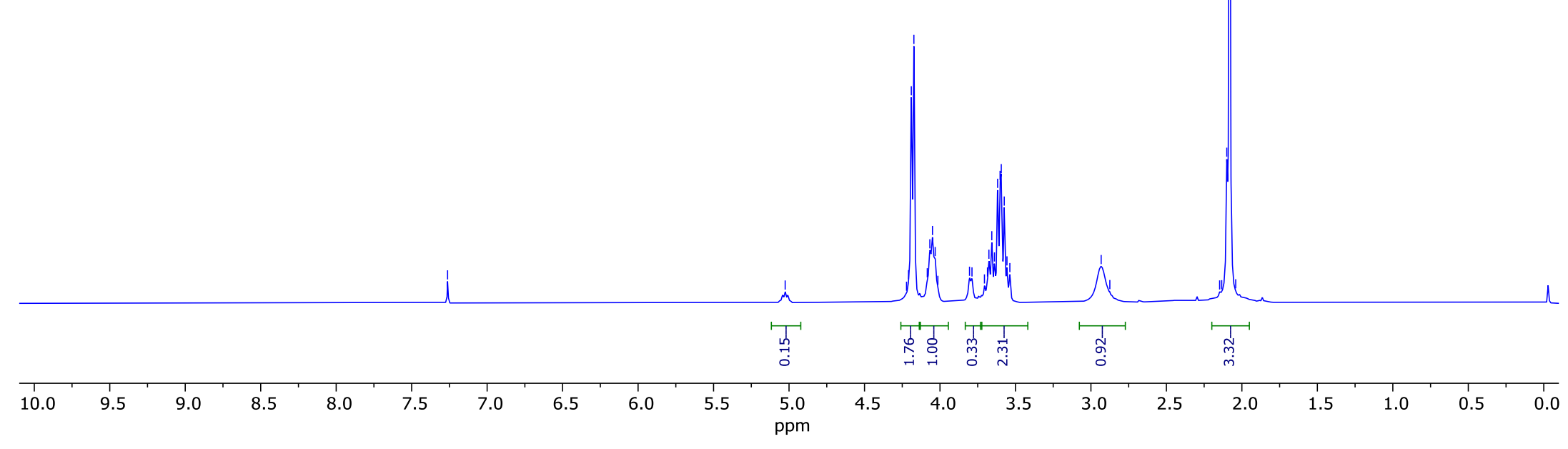


Figure S43: ${ }^{13} \mathrm{C}\left\{{ }^{1} \mathrm{H}\right\}$ NMR spectrum of 3-chloro-2-hydroxypropyl acetate ((rac)-1a) and 1-chloro-3-hydroxypropan-2-yl acetate ((rac)-1b. ((rac)1a): $\left((\mathrm{rac})-\mathbf{1 b}=7.6: 1\left(\mathrm{CDCl}_{3}, 75 \mathrm{MHz}\right)\right.$.<smiles>CC(=O)OCC(O)CCl</smiles>

(rac)-1a<smiles>CC(=O)OC(CO)CCl</smiles>

(rac)-1b

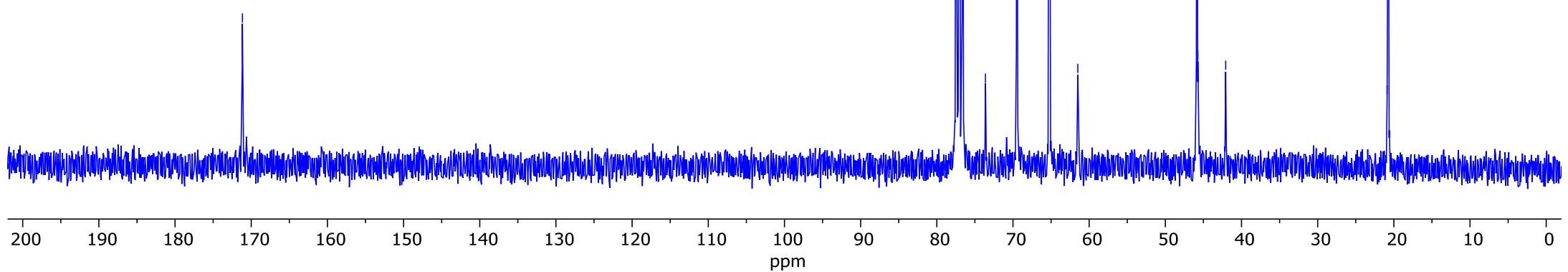


Figure S44: ${ }^{1} \mathrm{H}$ NMR spectrum of 3-chloropropane-1,2-diyl diacetate (( $\left.\left.r a c\right)-5\right)\left(\mathrm{CDCl}_{3}, 300 \mathrm{MHz}\right)$.

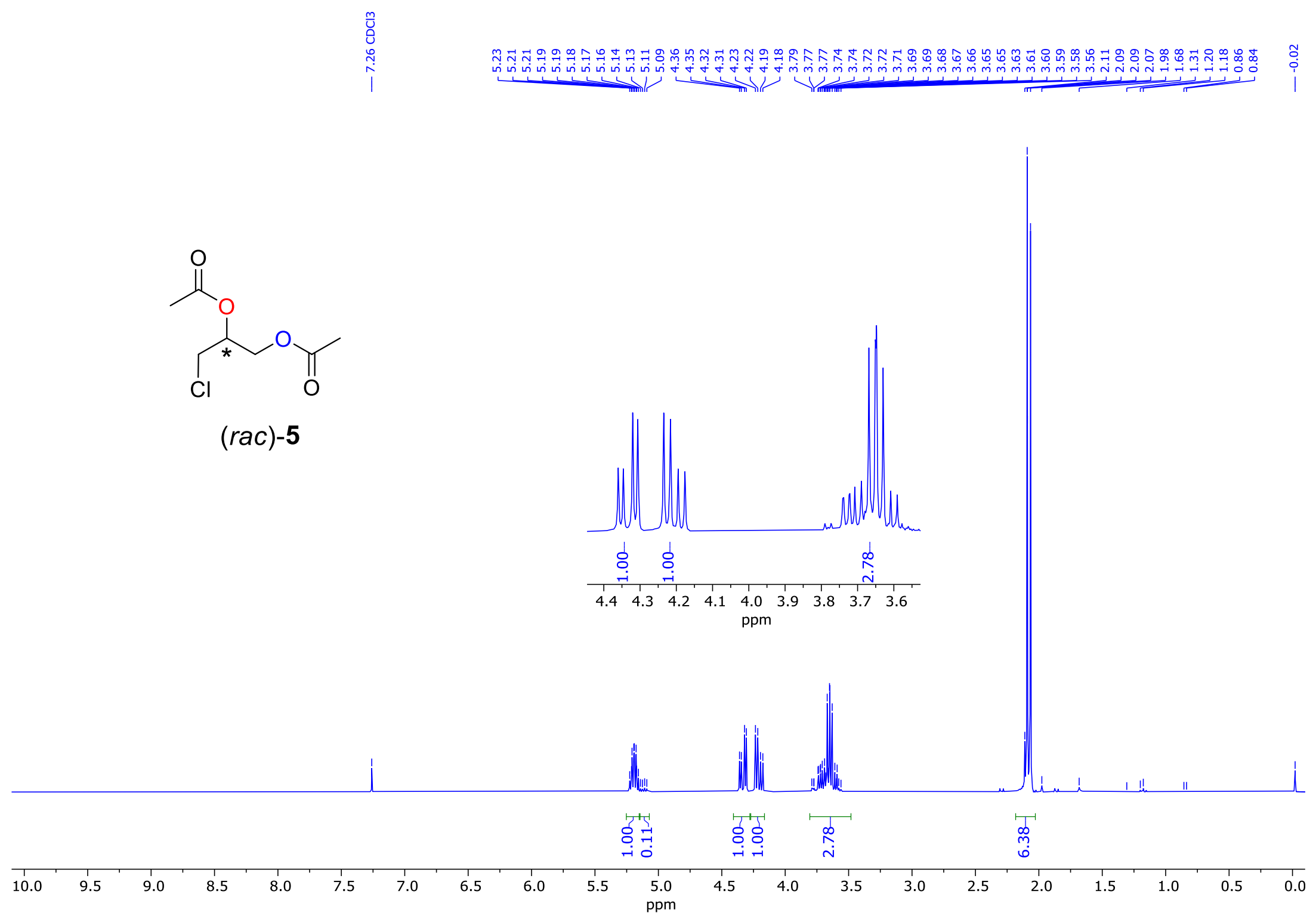


Figure S45: ${ }^{13} \mathrm{C}\left\{{ }^{1} \mathrm{H}\right\}$ NMR spectrum of 3-chloropropane-1,2-diyl diacetate ((rac)-5) $\left(\mathrm{CDCl}_{3}, 75 \mathrm{MHz}\right)$.

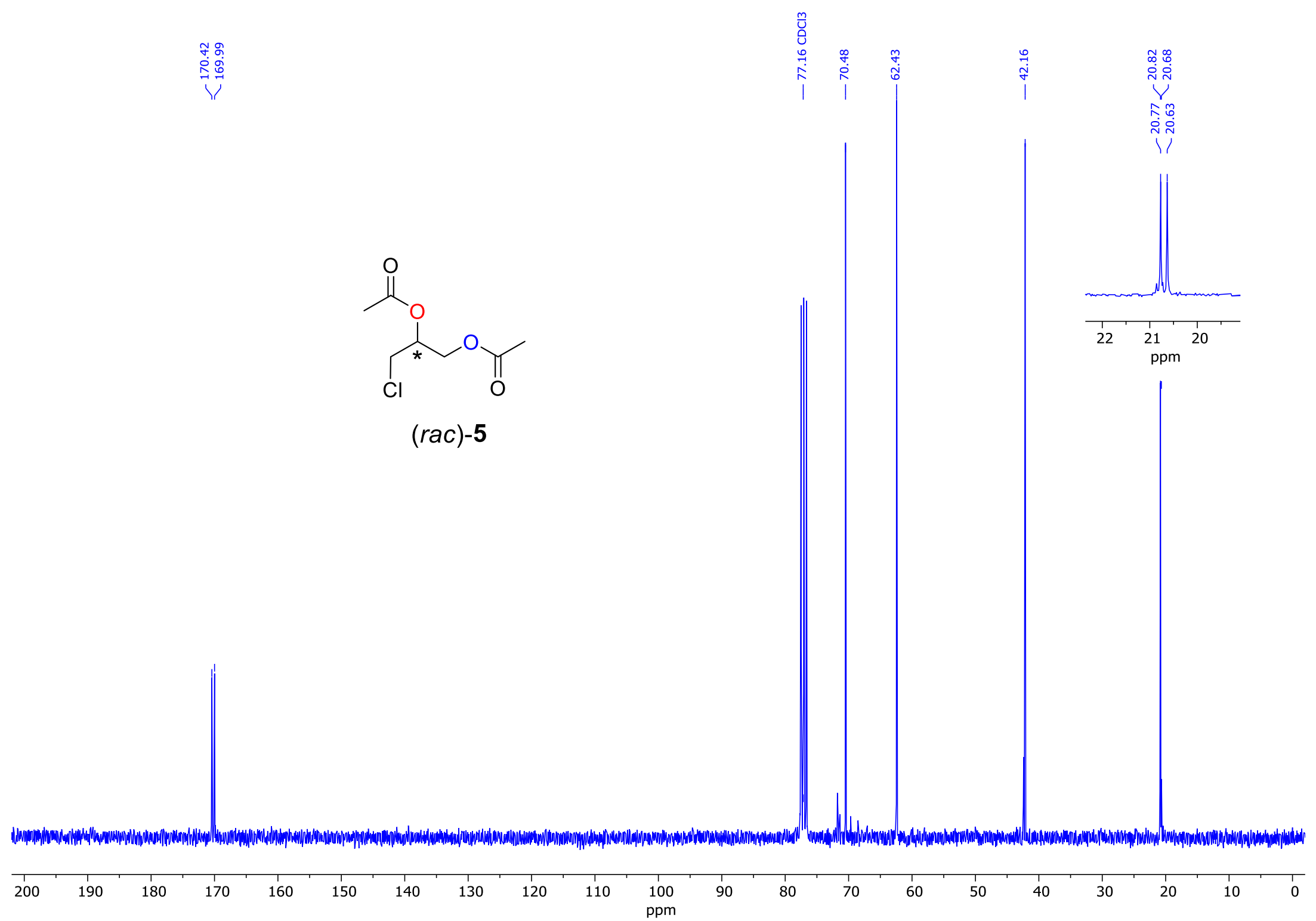


Figure S46: ${ }^{1} \mathrm{H}$ NMR spectrum 3-chloropropane-1,2-diyl dibenzoate ((rac)-7) $\left(\mathrm{CDCl}_{3}, 300 \mathrm{MHz}\right)$.

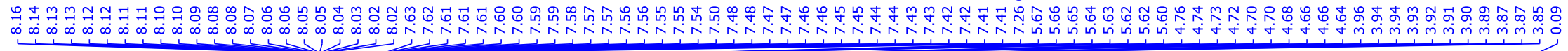

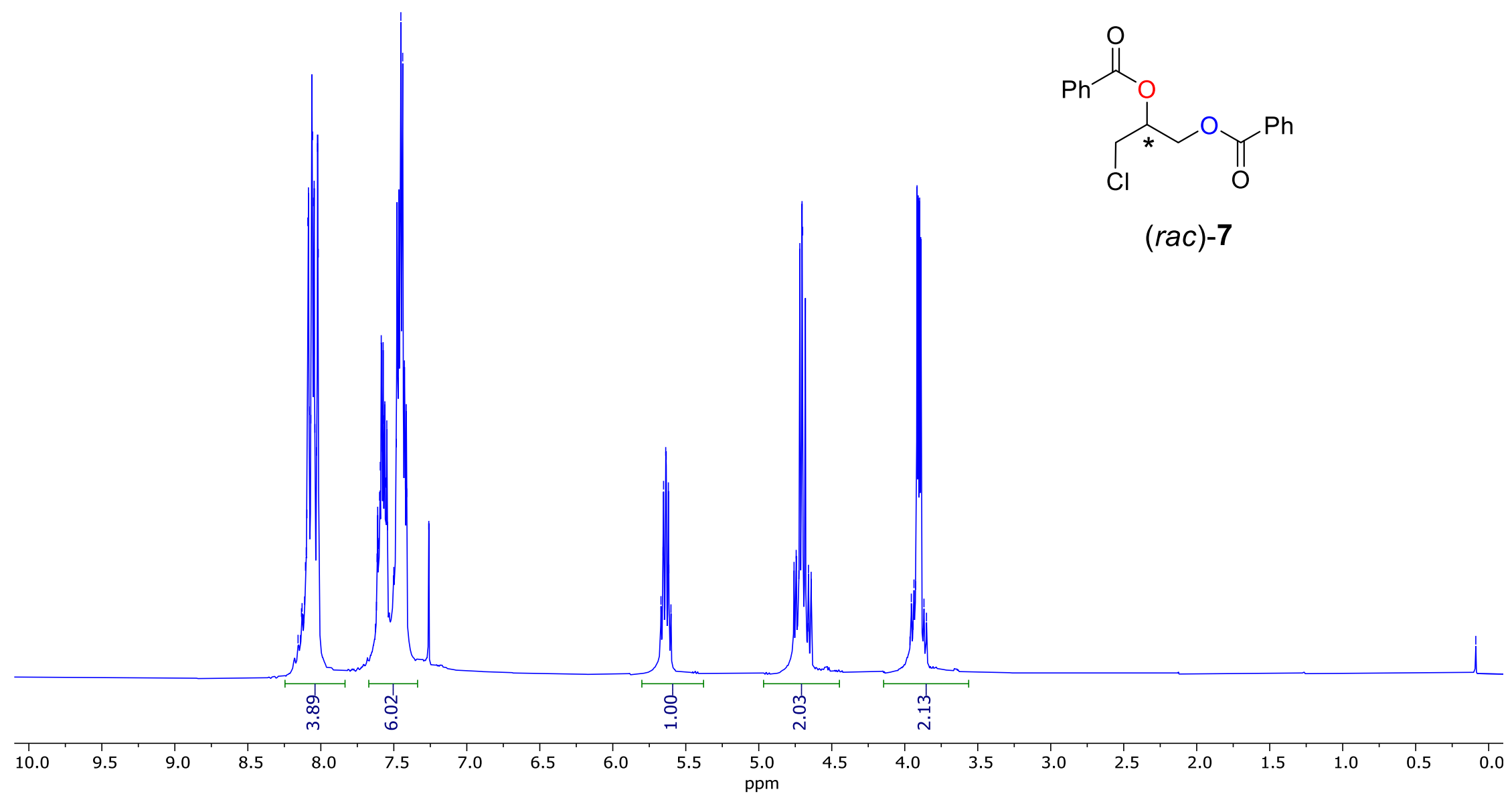


Figure S47: ${ }^{13} \mathrm{C}\left\{{ }^{1} \mathrm{H}\right\}$ NMR spectrum of 3-chloropropane-1,2-diyl dibenzoate ((rac)-7) $\left(\mathrm{CDCl}_{3}, 75 \mathrm{MHz}\right)$.

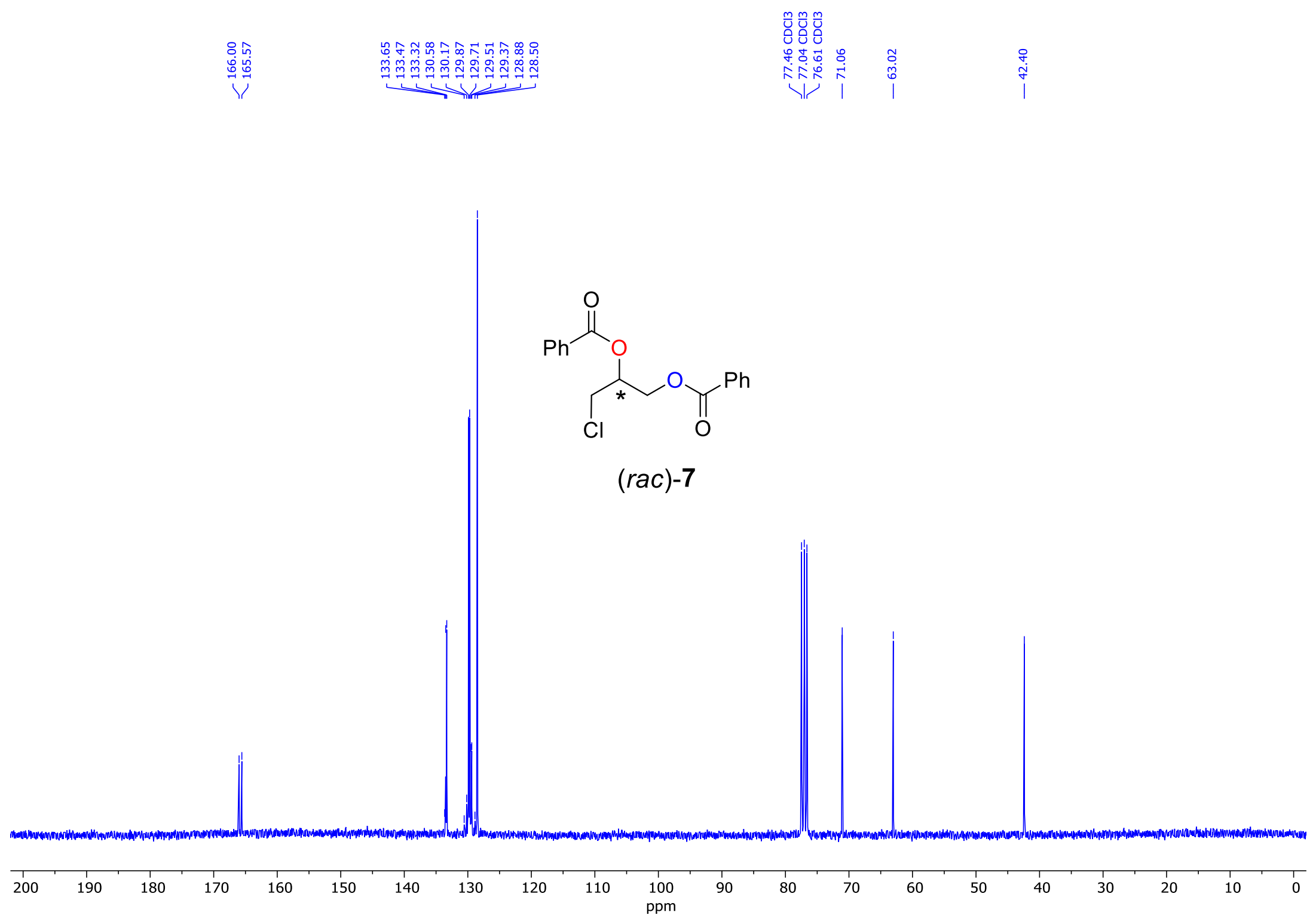


Figure S48: ${ }^{1} \mathrm{H}$ NMR spectrum of 3-(1,3-dioxoisoindolin-2-yl)propane-1,2-diyl diacetate (( $\left.\left.\mathrm{rac}\right)-11 \mathrm{a}\right)\left(\mathrm{CDCl}_{3}, 300 \mathrm{MHz}\right)$

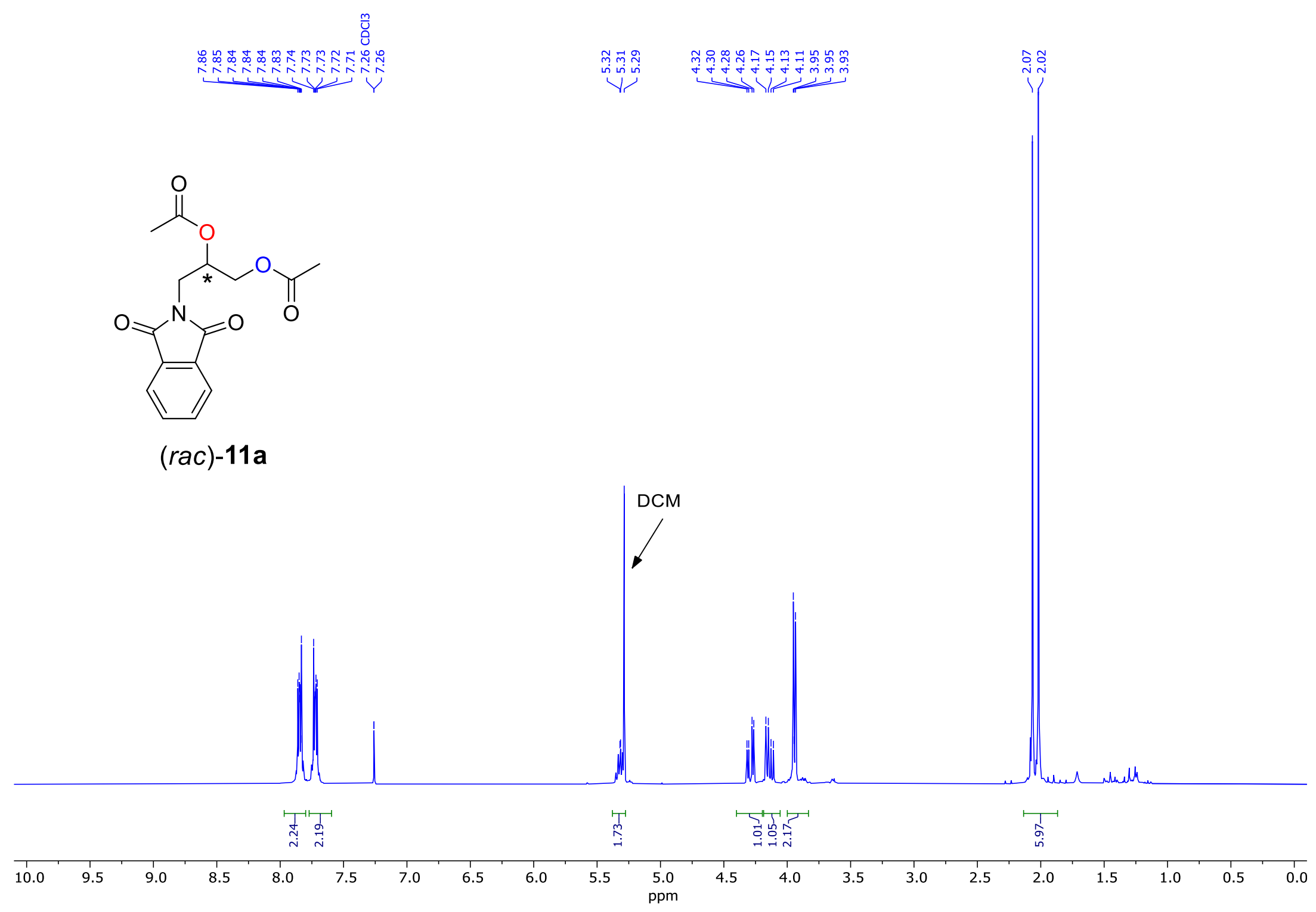


Figure S49: ${ }^{13} \mathrm{C}\left\{{ }^{1} \mathrm{H}\right\}$ NMR spectrum of 3-(1,3-dioxoisoindolin-2-yl)propane-1,2-diyl diacetate ((rac)-11a) $\left(\mathrm{CDCl}_{3}, 75 \mathrm{MHz}\right)$.

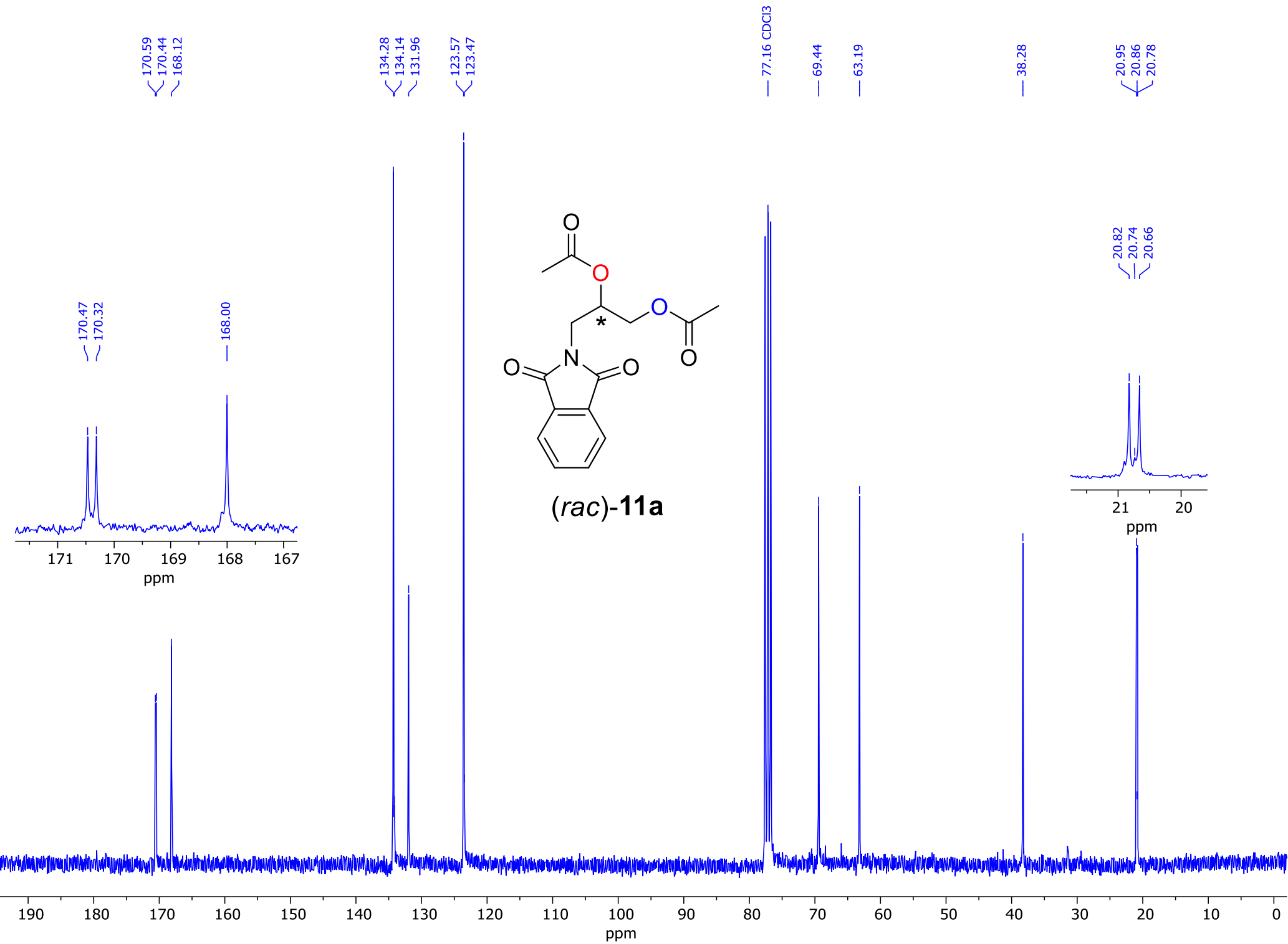


Figure S50: ${ }^{1} \mathrm{H}$ NMR spectrum of purified 2-hydroxy-3-phenoxypropyl acetate ((rac)-13e) $\left(\mathrm{CDCl}_{3}, 300 \mathrm{MHz}\right)$.

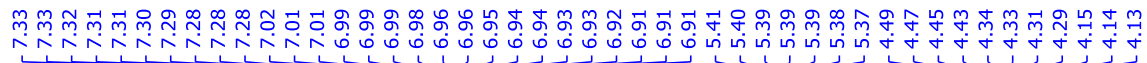

计

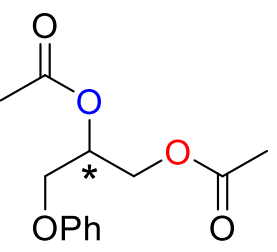

(rac)-13e

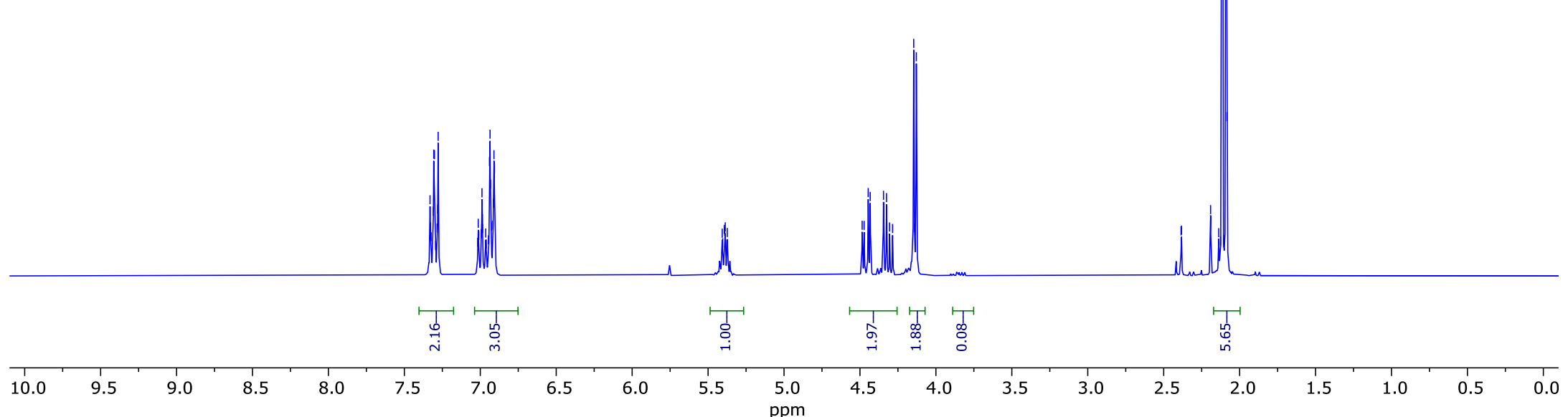


Figure S51: ${ }^{13} \mathrm{C}\left\{{ }^{1} \mathrm{H}\right\}$ NMR spectrum of purified 2-hydroxy-3-phenoxypropyl acetate ((rac)-13e) $\left(\mathrm{CDCl}_{3}, 75 \mathrm{MHz}\right)$.
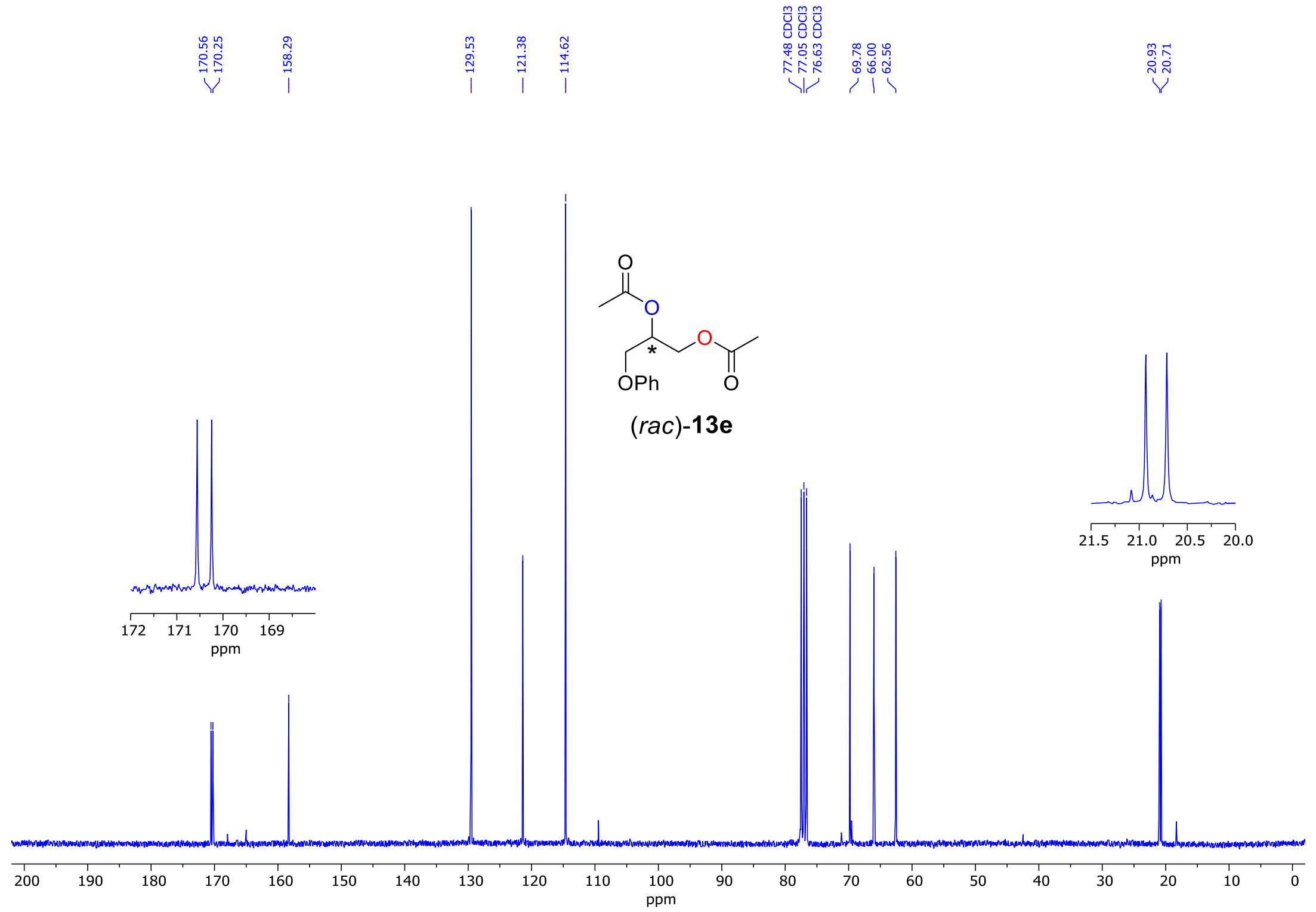
Figure S52: ${ }^{1} \mathrm{H}$ NMR spectrum of 3-phenoxypropane-1,2-diyl bis(2,2-dimethylpropanoate) ((rac)-15) (CDCl $\left.3,300 \mathrm{MHz}\right)$.
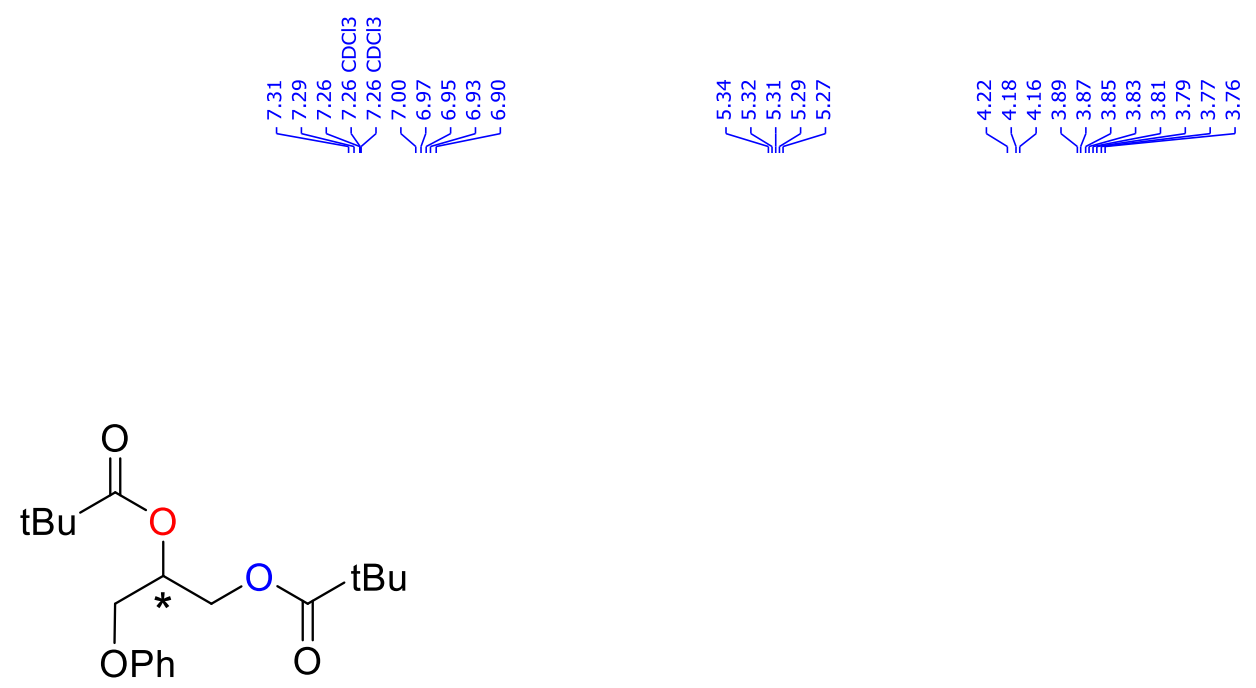

(rac)-15

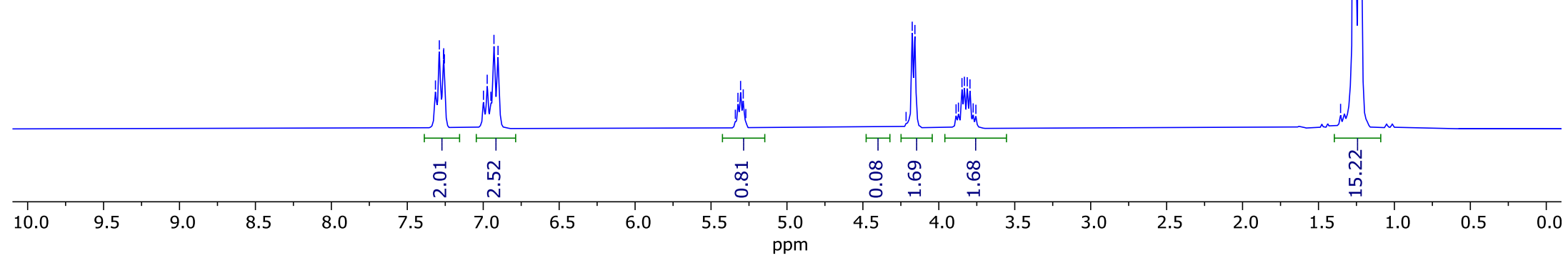


Figure S53: ${ }^{13} \mathrm{C}\left\{{ }^{1} \mathrm{H}\right\}$ NMR spectrum of 3-phenoxypropane-1,2-diyl bis(2,2-dimethylpropanoate) ((rac)-15) $\left(\mathrm{CDCl}_{3}, 75 \mathrm{MHz}\right)$.

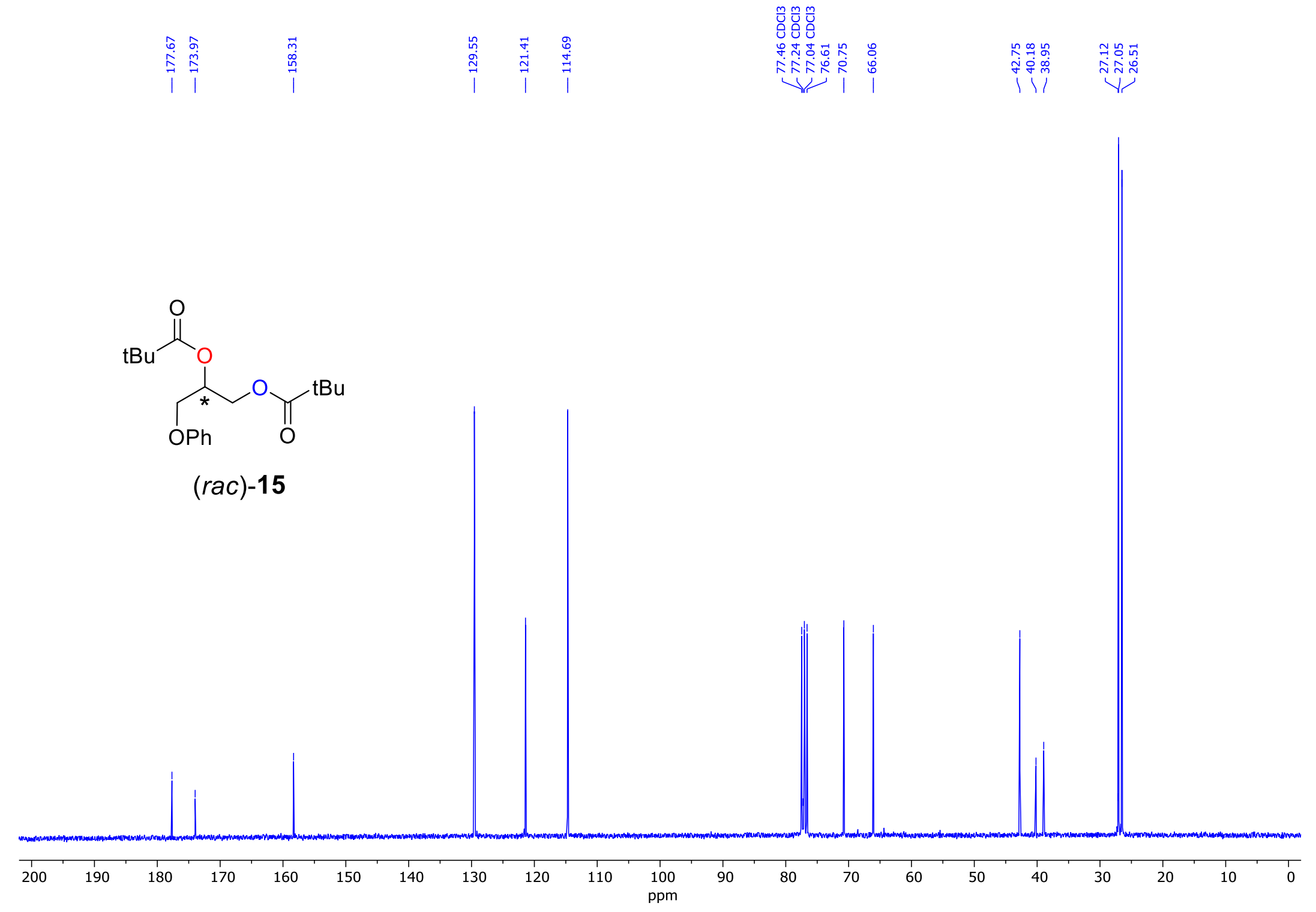


Figure S54: ${ }^{1} \mathrm{H}$ NMR spectrum of 3-(allyloxy)propane-1,2-diyl diacetate $17\left(\mathrm{CDCl}_{3}, 300 \mathrm{MHz}\right)$.
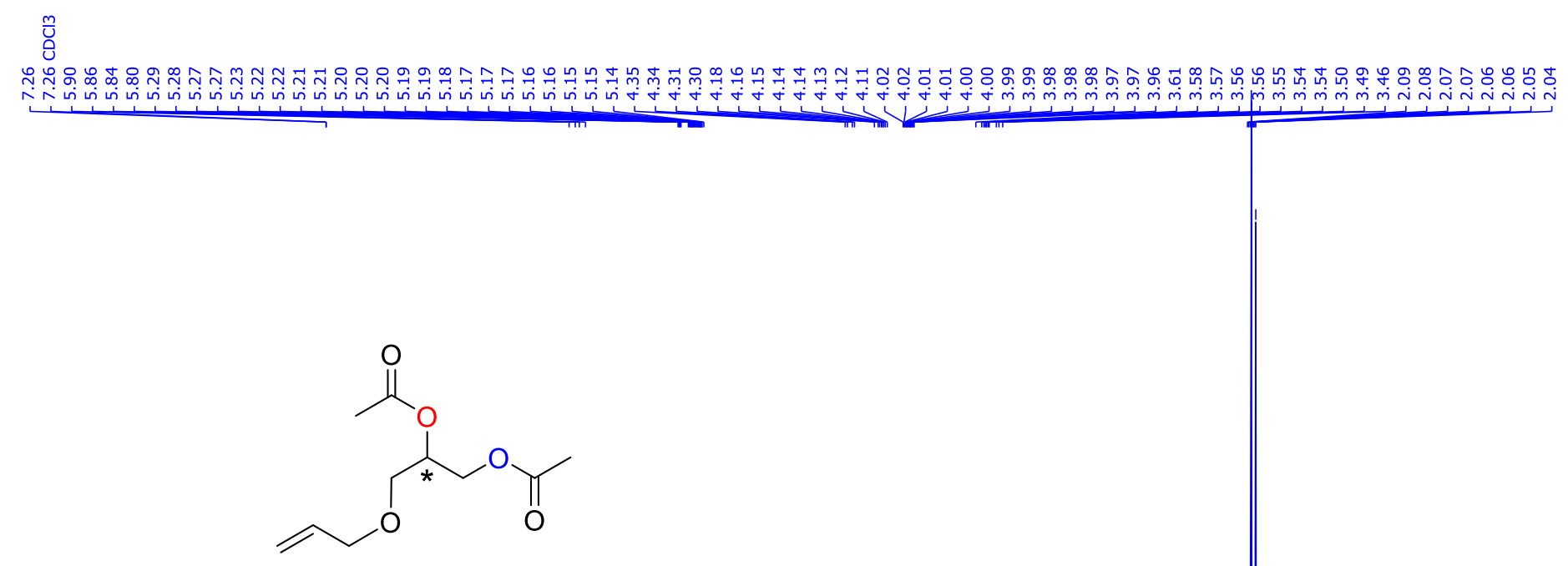

(rac)-17

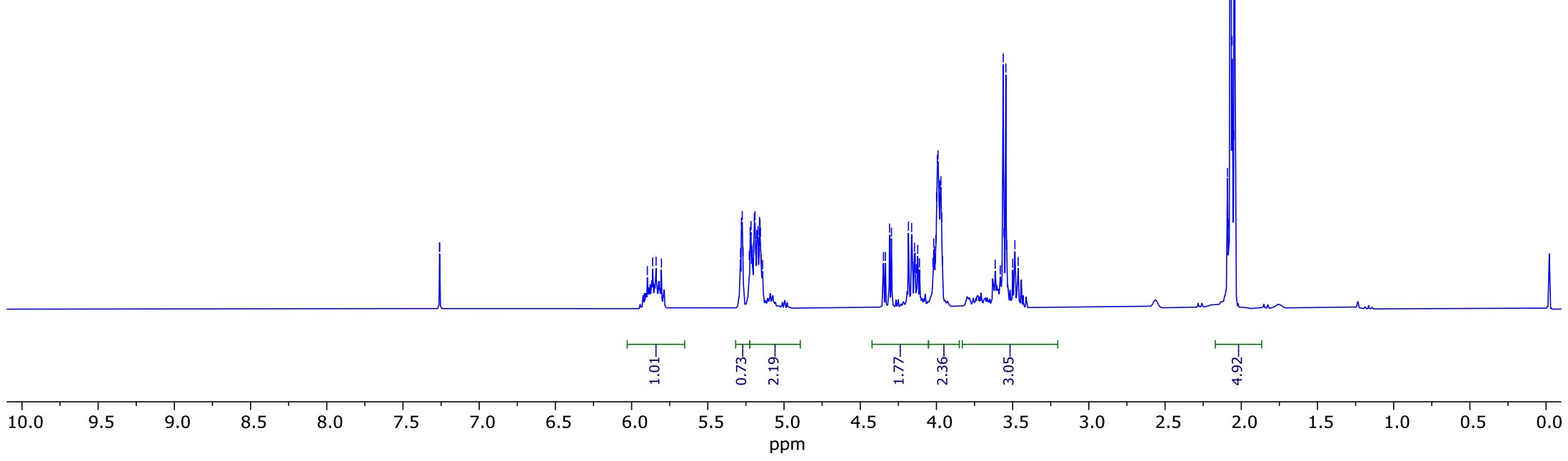


Figure S55: ${ }^{13} \mathrm{C}\left\{{ }^{1} \mathrm{H}\right\}$ NMR 3-(allyloxy)propane-1,2-diyl diacetate $17\left(\mathrm{CDCl}_{3}, 75 \mathrm{MHz}\right)$.
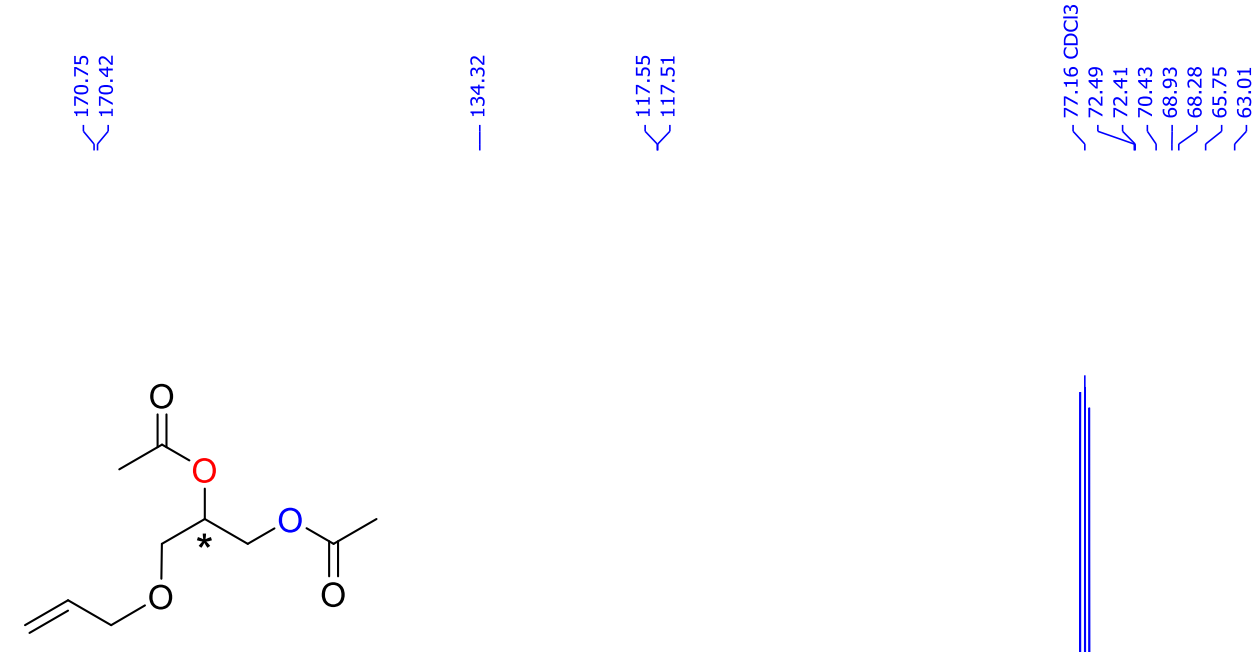

(rac)-17

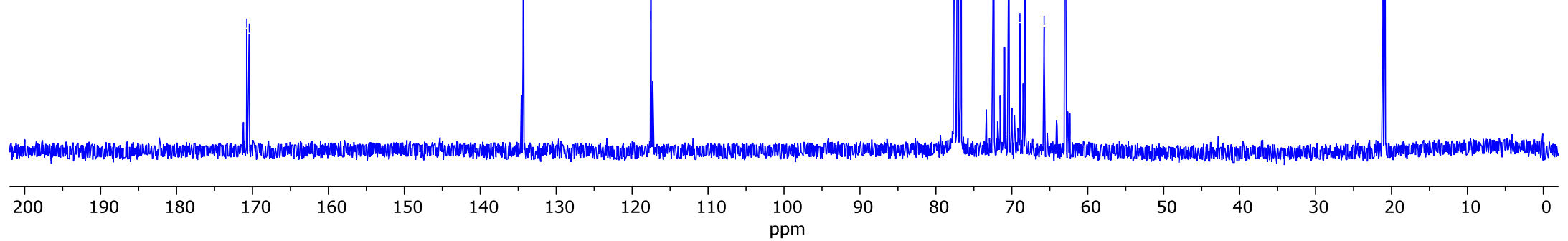


Figure S56: ${ }^{1} \mathrm{H}$ NMR spectrum of 2-hydroxy-3-phenoxypropyl acetate ((rac)-13a) $\left(<5 \%((\mathrm{rac})-\mathbf{1 3 c})\left(\mathrm{CDCl}_{3}, 300 \mathrm{MHz}\right)\right.$.

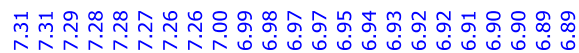

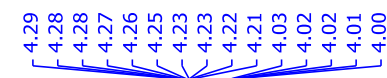<smiles>CC(=O)OCC(O)Cc1ccccc1</smiles>

$(r a c)-13 a$

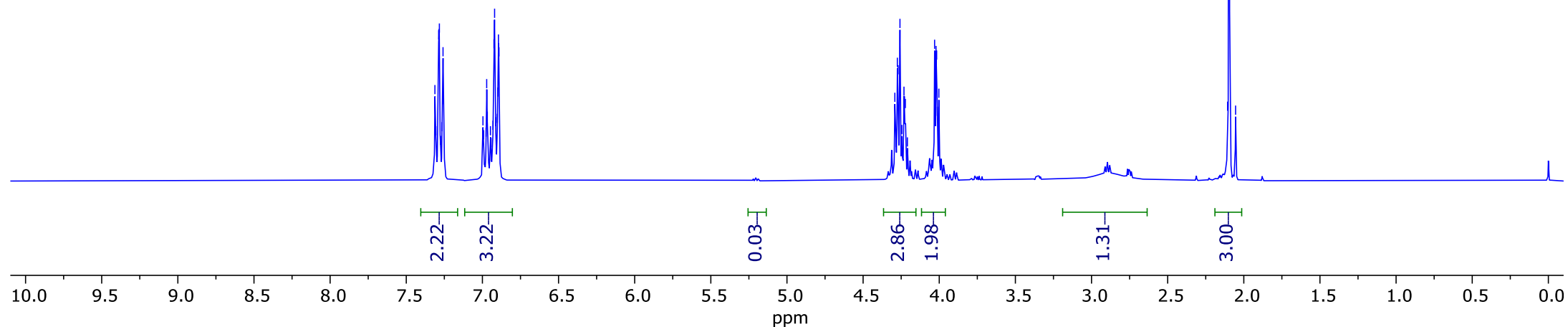


Figure S57: ${ }^{13} \mathrm{C}\left\{{ }^{1} \mathrm{H}\right\}$ NMR spectrum of 2-hydroxy-3-phenoxypropyl acetate $((\mathrm{rac})-\mathbf{1 3 a})\left(<5 \%((\mathrm{rac})-\mathbf{1 3 c})\left(\mathrm{CDCl}_{3}, 300 \mathrm{MHz}\right)\right.$.

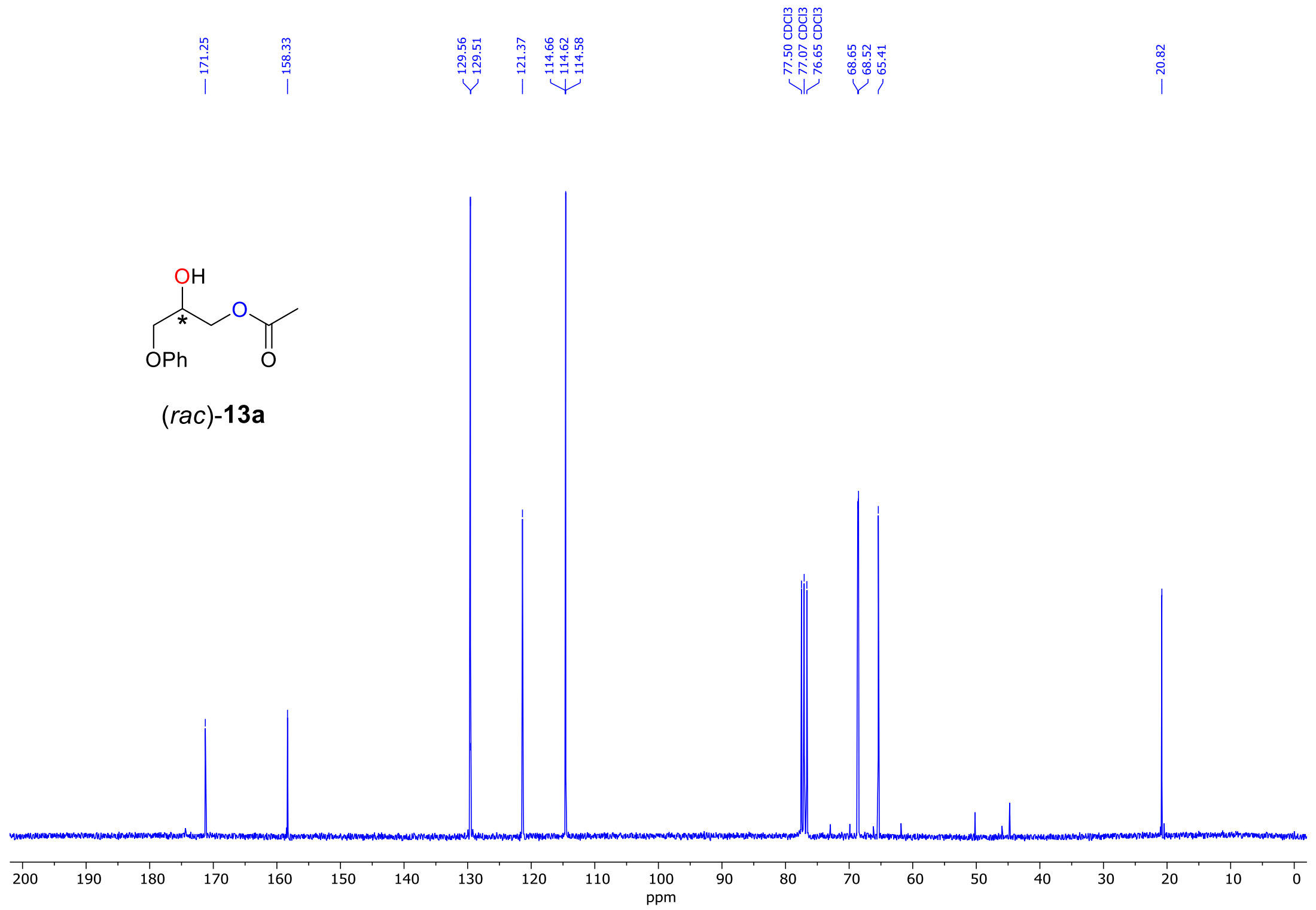


Figure S58: ${ }^{1} \mathrm{H}$ NMR spectrum of cation 13b exchange with EtOAc to produce a crude 2-hydroxy-3-phenoxypropyl acetate $((\mathrm{rac})-\mathbf{1 3 e})\left(\mathrm{CDCl}_{3}\right.$, $300 \mathrm{MHz}$ ).

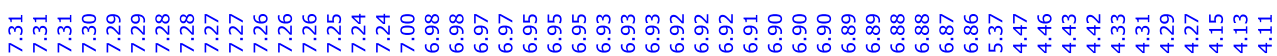

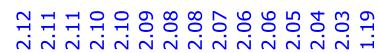<smiles>CC(=O)OC[C@H](Cc1ccccc1)OC(C)=O</smiles>

(rac)-13e

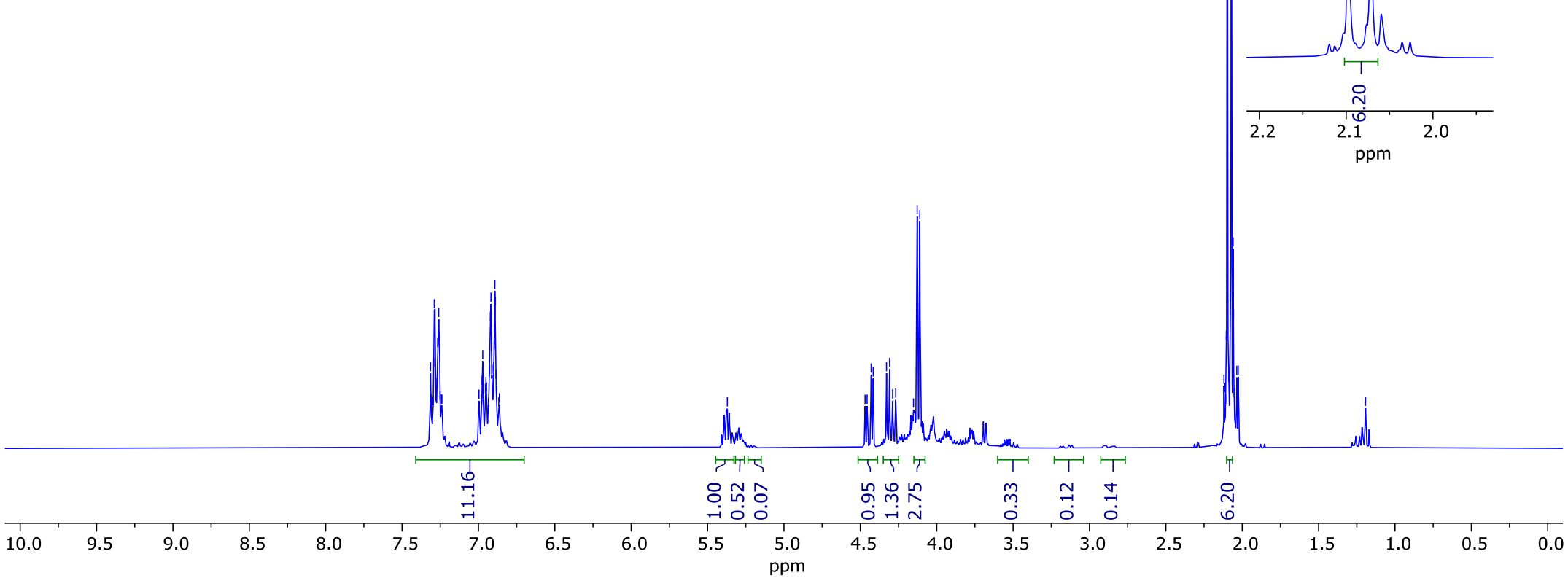


Figure S59: ${ }^{13} \mathrm{C}\left\{{ }^{1} \mathrm{H}\right\}$ NMR spectrum of cation $\mathbf{1 3 b}$ exchange with EtOAc to crude 2-hydroxy-3-phenoxypropyl acetate $((\mathrm{rac})-\mathbf{1 3 e})\left(\mathrm{CDCl}{ }_{3}, 75\right.$ $\mathrm{MHz}$ ).

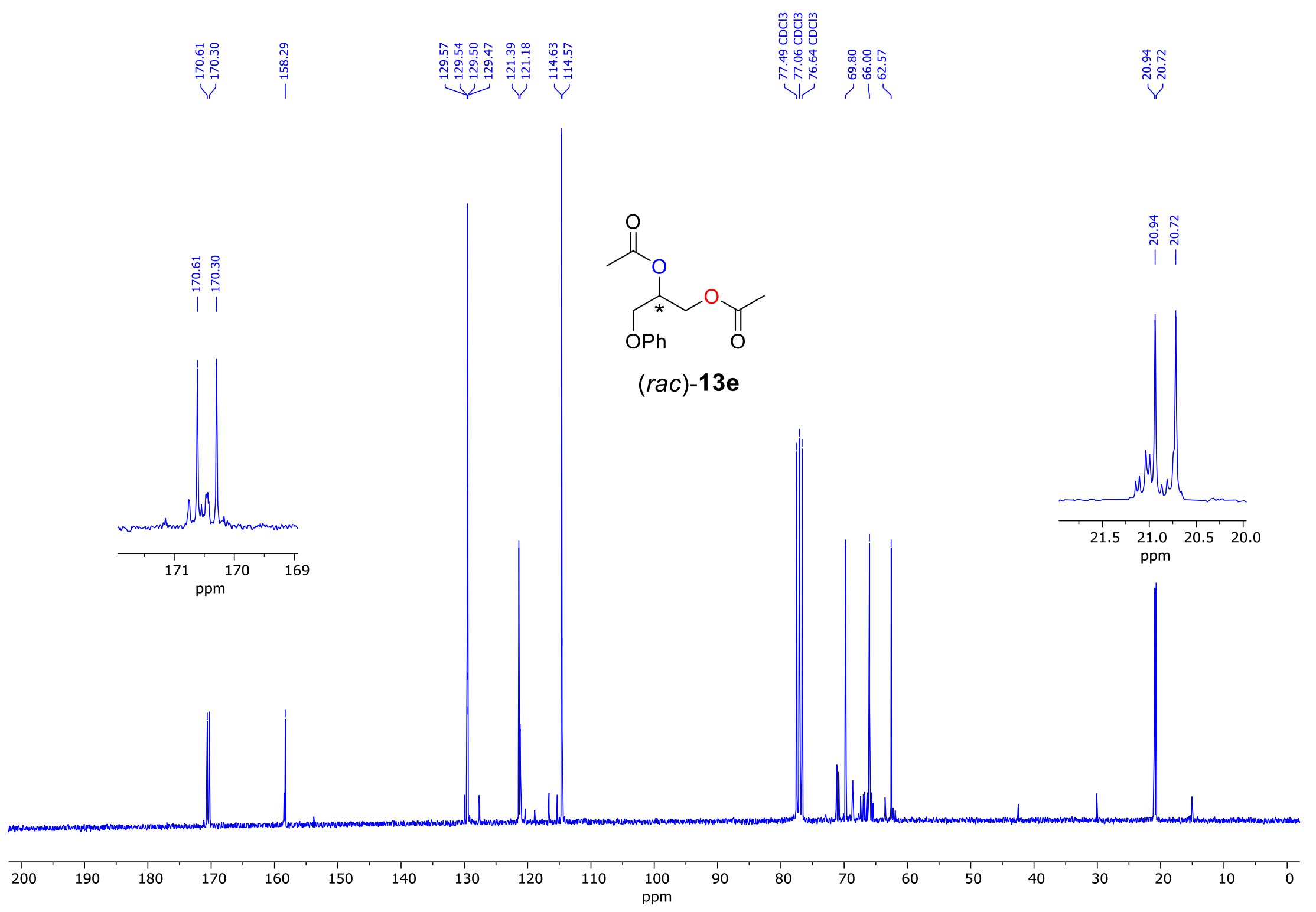


Figure S60: ${ }^{13} \mathrm{C}\left\{{ }^{1} \mathrm{H}\right\}$ NMR overlay of purified 2-hydroxy-3-phenoxypropyl acetate ((rac)-13e) (top, maroon spectrum) versus crude cation exchange (bottom, blue spectrum) $\left(\mathrm{CDCl}_{3}, 75 \mathrm{MHz}\right)$.

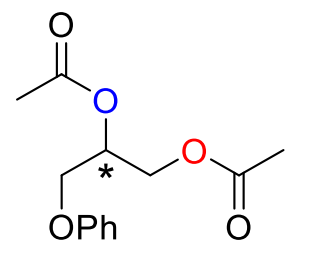

$($ rac)-13e

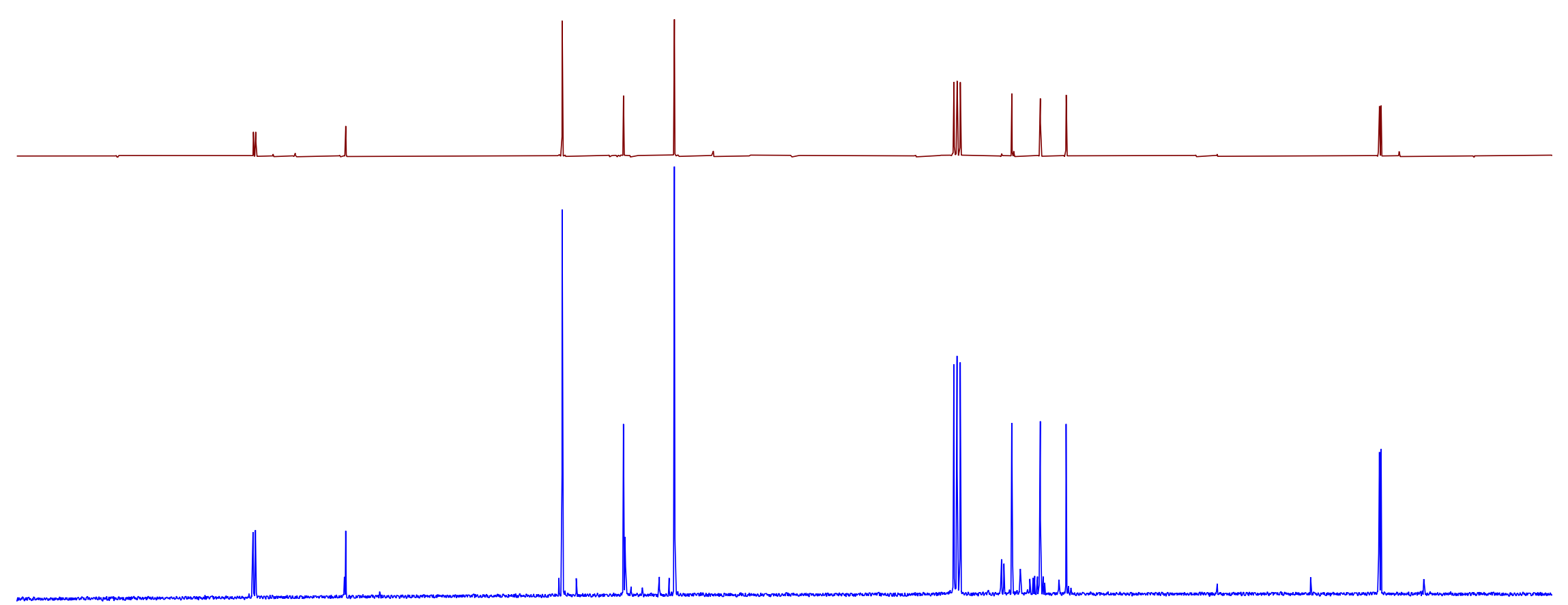


Figure S61: Chiral HPLC of $(R, S)$-1-acetamido-3-chloropropan-2-yl benzoate ((rac)-6) vs. $(R)$-1-acetamido-3-chloropropan-2-yl benzoate $((R)$ 6b).

Resolution of enantiomers: DICAL Chiralcel® OJ-H, 94-6 Hexane-IPA, $1.0 \mathrm{~mL} / \mathrm{min}, 254 \mathrm{~nm}, \mathrm{RT} 1$ (major) = $24.3 \mathrm{~min}, \mathrm{RT} 2$ (minor) =31.3 min. er 98:2 (Isocratic).
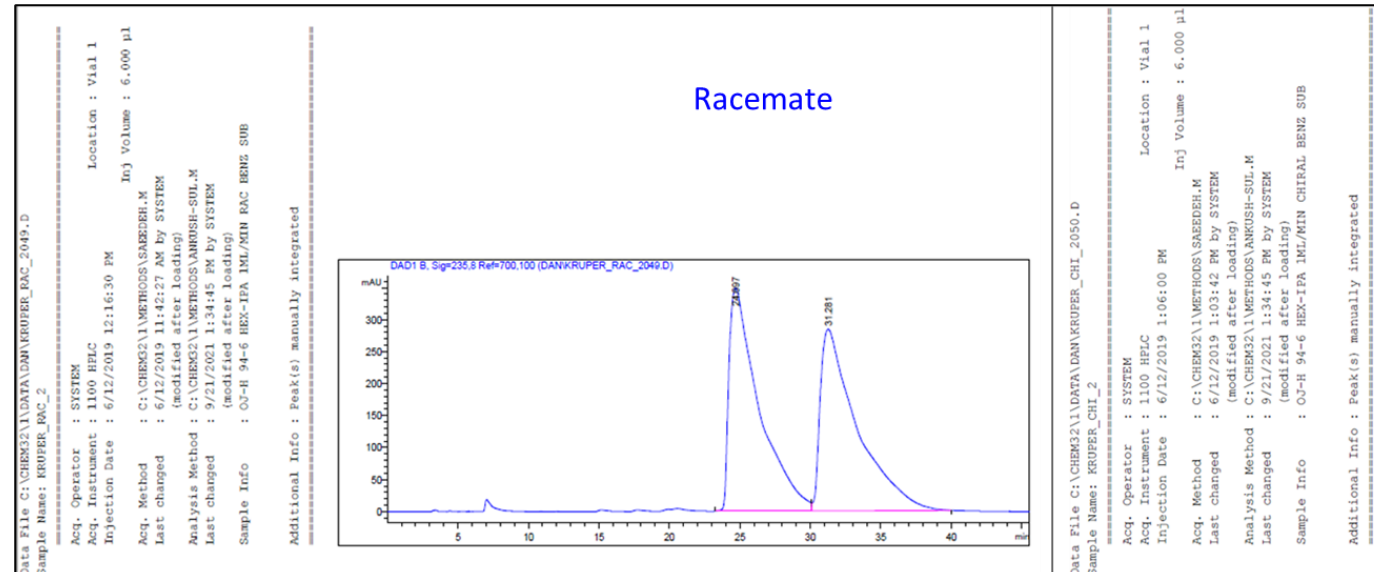

Single

enantiomer

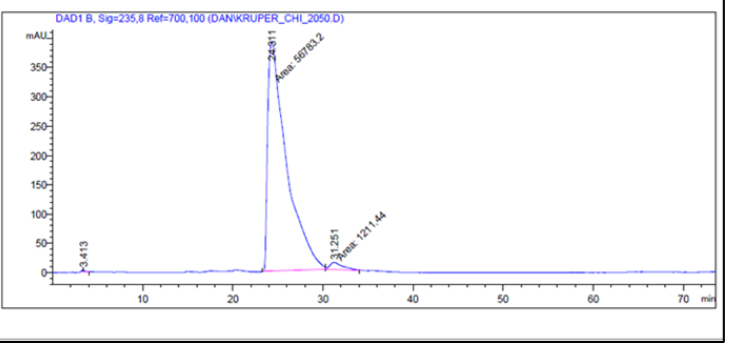


Figure S62. Chiral HPLC of $(R, S)$-1-acetamido-3-(1,3-dioxoisoindolin-2-yl)propan-2-yl acetate ((rac)-10a) vs. $(R)$-1-acetamido-3-(1,3dioxoisoindolin-2-yl)propan-2-yl acetate $((R)-\mathbf{1 0 b})$ (Isocratic).

Resolution of enantiomers: DICAL Chiralcel ${ }^{\circledR}$ OJ-H, 90-10 Hexane-IPA, $1.0 \mathrm{~mL} / \mathrm{min}, 230 \mathrm{~nm}, \mathrm{RT} 1$ (minor) = 58.9 min, RT2 (major) =67.2 min. $\operatorname{er} 99: 1$

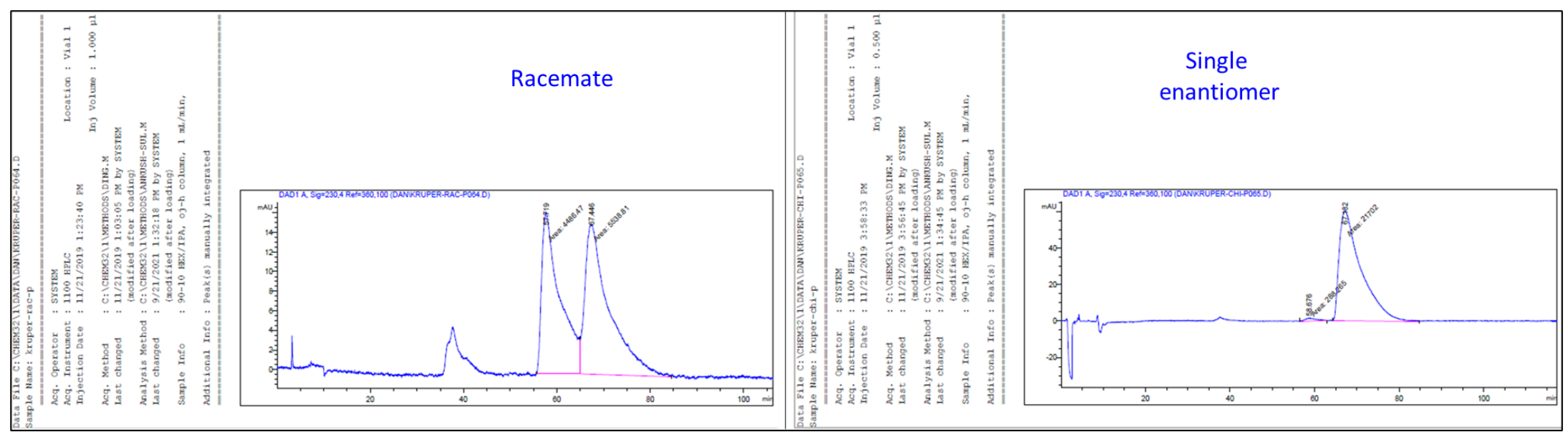


Figure S63: DSC of amidoester (S)-4b.

Sample: S-Epi amide pure crystal Size: $6.3000 \mathrm{mg}$

Method: Dan'sMethod\#2

DSC

File: C:ITAIDatalDSCIKruperIS-Epi Amide.001

Run Date 13-Aug-2019 13:33

Instrument: DSC Q20 V24.11 Build 124

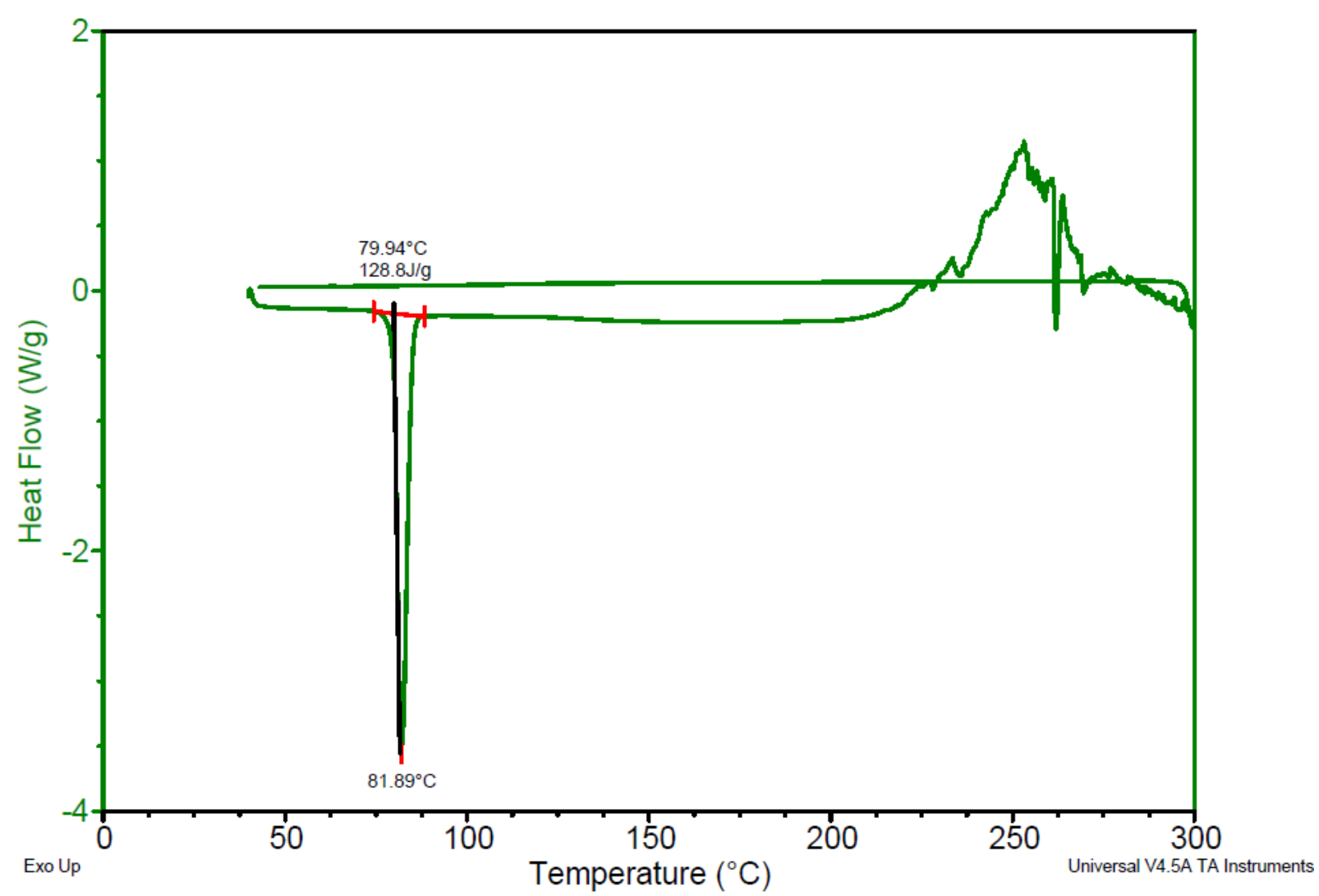


Figure S64: DSC of amidoester $(R)-\mathbf{1 0 b}$.

Sample: S-phthalimido amide pure crystal Size: $4.5000 \mathrm{mg}$

DSC

File: C:ITAIDataIDSCIKruperIS-phthalimide.001

Run Date: 13-Aug-2019 11:56

Instrument: DSC Q20 V24.11 Build 124

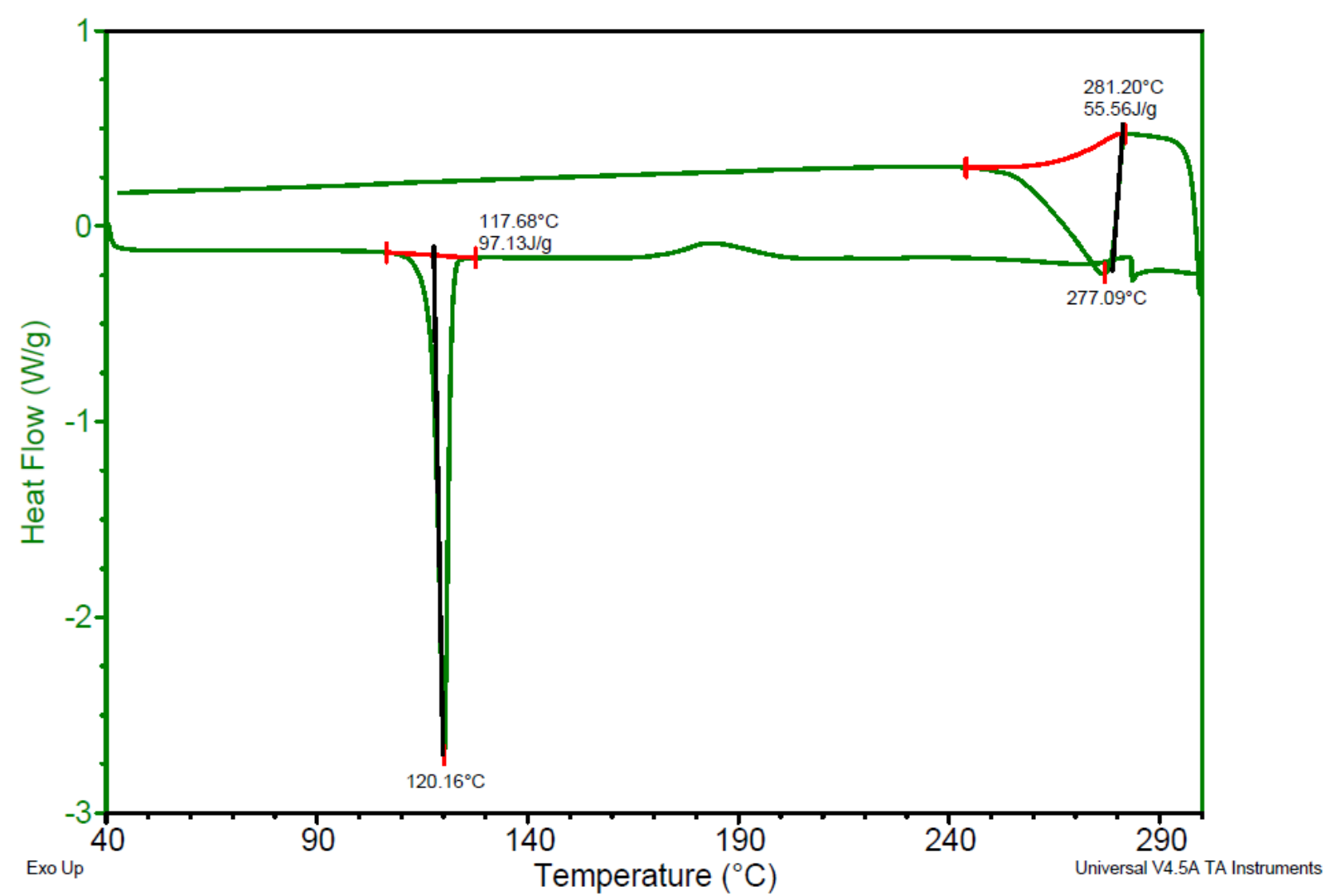


Figure S65: Molecular Structures of (S)-4b (CCDC Code: 2088283). Space group P $2{ }_{1} 2_{1} 2_{1}$. Thermal ellipsoids are shown at $50 \%$ probability

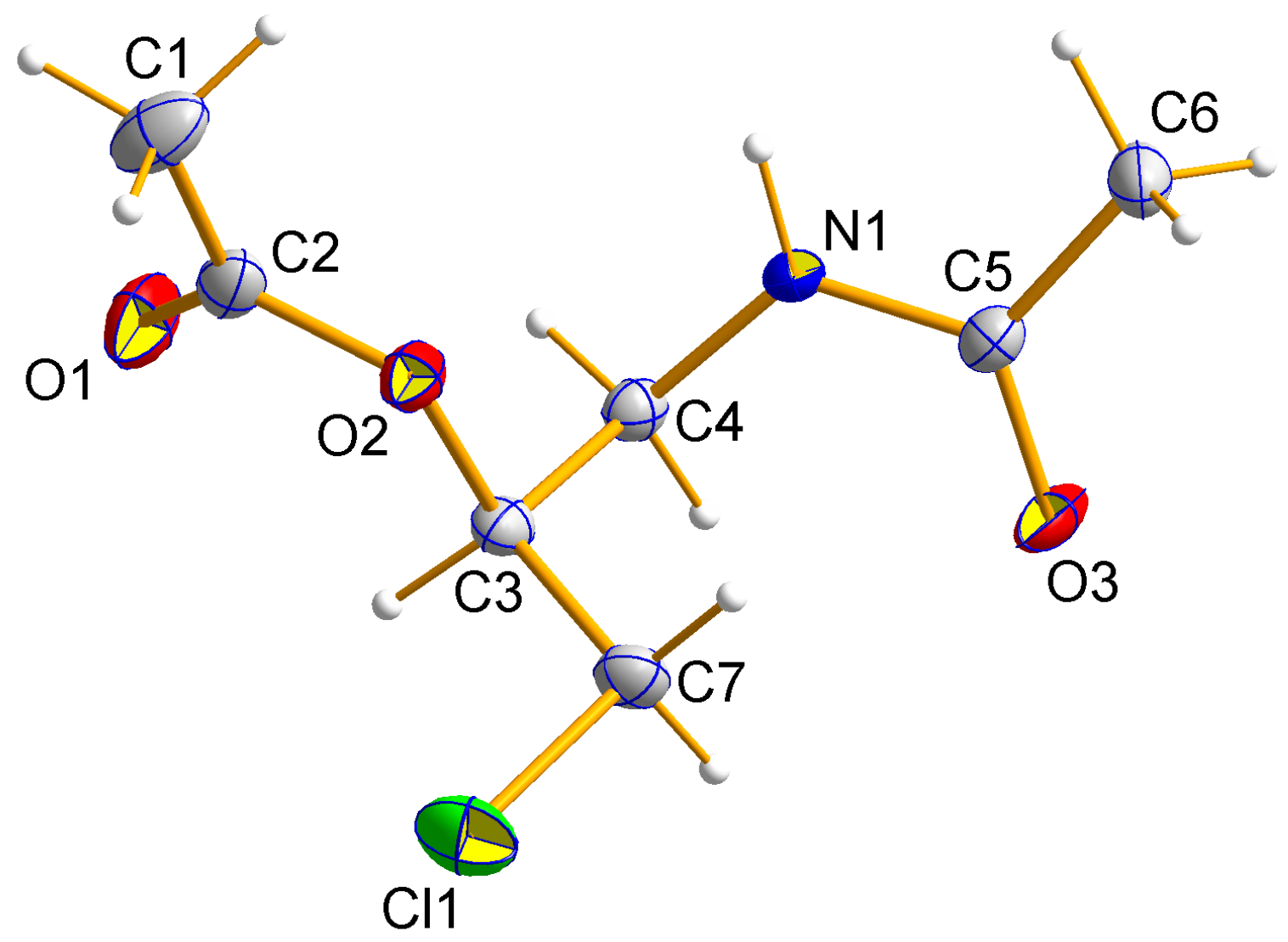


Figure S66: Molecular Structures of $(R)-\mathbf{4 c}$ (CCDC Code: 2088284). Space group P $2{ }_{1}{ }_{1}{ }_{2}{ }_{1}$. Thermal ellipsoids are shown at $50 \%$ probability.

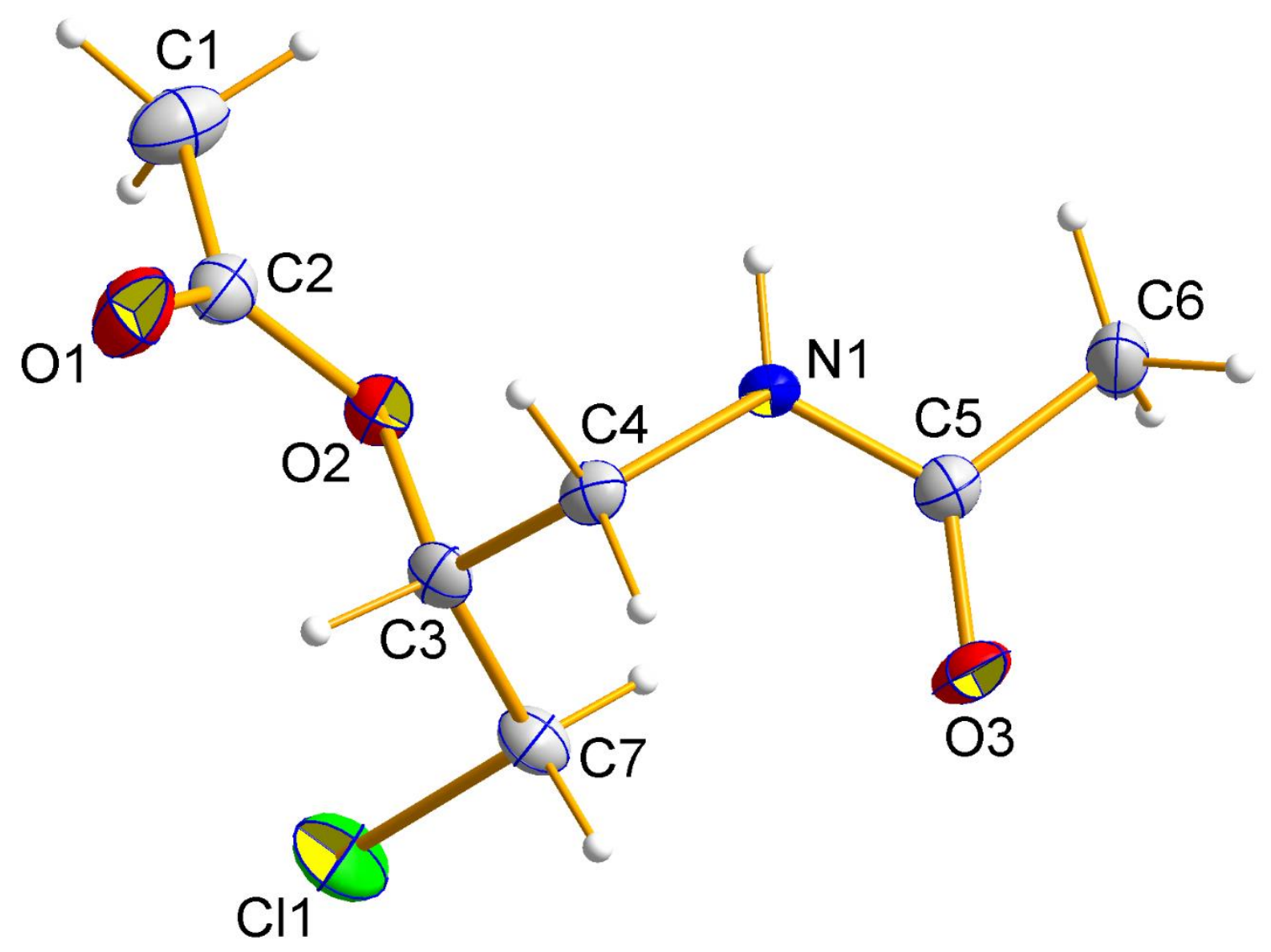


Figure S67: Molecular Structures of $(R)$-10b (CCDC Code: 2088285). Thermal ellipsoids are shown at 50\% probability.

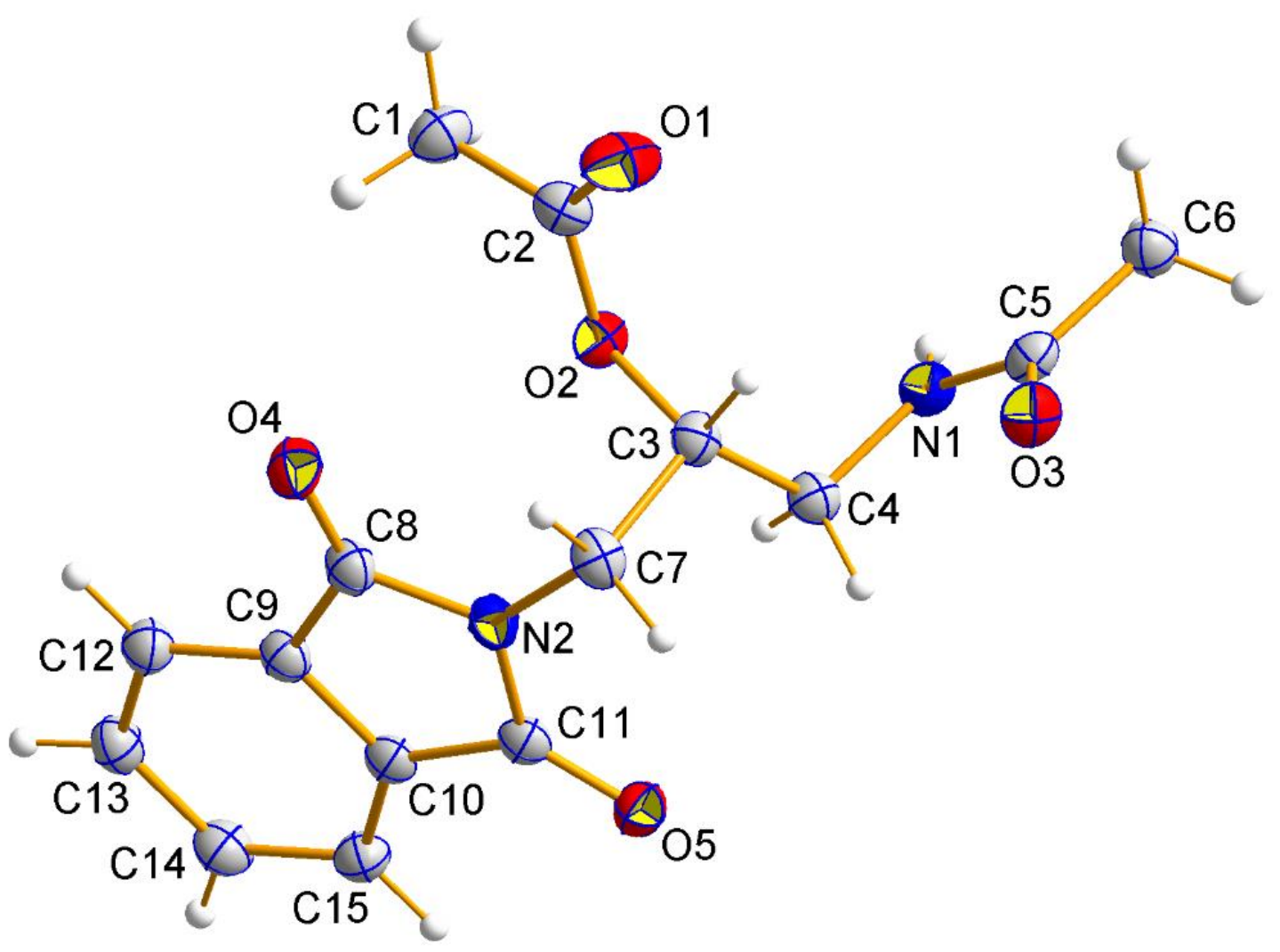


Table 1. Crystal data and structure refinement for (S)-4b (CCDC Code: 2088283).

\begin{tabular}{|c|c|c|}
\hline Empirical formula & \multicolumn{2}{|l|}{$\mathrm{C}_{7} \mathrm{H}_{12} \mathrm{ClNO}_{3}$} \\
\hline Formula weight & \multicolumn{2}{|l|}{193.63} \\
\hline Temperature & \multicolumn{2}{|l|}{$100(2) \mathrm{K}$} \\
\hline Wavelength & \multicolumn{2}{|l|}{$0.71073 \AA$} \\
\hline Crystal system & \multicolumn{2}{|l|}{ Orthorhombic } \\
\hline Space group & \multicolumn{2}{|l|}{$\mathrm{P} 2{ }_{1}{ }_{1} 2_{1}$} \\
\hline \multirow[t]{3}{*}{ Unit cell dimensions } & $a=8.4293(5) \AA$ & $\alpha=90^{\circ}$. \\
\hline & $\mathrm{b}=9.5216(5) \AA$ & $\beta=90^{\circ}$. \\
\hline & $\mathrm{c}=12.3239(7) \AA$ & $\gamma=90^{\circ}$ \\
\hline Volume & \multicolumn{2}{|l|}{$989.12(10) \AA^{3}$} \\
\hline Z & \multicolumn{2}{|l|}{4} \\
\hline Density (calculated) & \multicolumn{2}{|l|}{$1.300 \mathrm{Mg} / \mathrm{m}^{3}$} \\
\hline Absorption coefficient & \multicolumn{2}{|l|}{$0.357 \mathrm{~mm}^{-1}$} \\
\hline $\mathrm{F}(000)$ & \multicolumn{2}{|l|}{408} \\
\hline Crystal size & \multicolumn{2}{|c|}{$0.250 \times 0.210 \times 0.180 \mathrm{~mm}^{3}$} \\
\hline Theta range for data collection & \multicolumn{2}{|l|}{2.703 to $27.493^{\circ}$. } \\
\hline Index ranges & \multicolumn{2}{|c|}{$-10<=\mathrm{h}<=10,-12<=\mathrm{k}<=11,-16<=\mathrm{l}<=12$} \\
\hline Reflections collected & \multicolumn{2}{|l|}{5345} \\
\hline Independent reflections & \multicolumn{2}{|c|}{$2211[\mathrm{R}(\mathrm{int})=0.0132]$} \\
\hline Completeness to theta $=25.242^{\circ}$ & \multicolumn{2}{|l|}{$100.0 \%$} \\
\hline Refinement method & \multicolumn{2}{|c|}{ Full-matrix least-squares on $\mathrm{F}^{2}$} \\
\hline Data / restraints / parameters & \multicolumn{2}{|l|}{$2211 / 0 / 111$} \\
\hline Goodness-of-fit on $\mathrm{F}^{2}$ & \multicolumn{2}{|l|}{1.090} \\
\hline Final $R$ indices [I>2sigma(I)] & \multicolumn{2}{|c|}{$\mathrm{R} 1=0.0224, \mathrm{wR} 2=0.0606$} \\
\hline
\end{tabular}


$\mathrm{R}$ indices (all data)

Absolute structure parameter

Largest diff. peak and hole
$\mathrm{R} 1=0.0235, \mathrm{wR} 2=0.0613$

$0.023(16)$

0.218 and -0.175 e. $\AA^{-3}$

$R 1=\sum\left(|| F_{o}|-| F_{c}||\right) / \sum\left|F_{0}\right| w R 2=\left[\sum\left[w\left(F_{o}^{2}-F_{c}^{2}\right)^{2}\right] / \sum\left[w\left(F_{o}^{2}\right)^{2}\right]\right]^{1 / 2}$

$S=\left[\sum\left[w\left(F_{o}{ }^{2}-F_{c}{ }^{2}\right)^{2}\right] /(n-p)\right]^{1 / 2} w=1 /\left[\sigma^{2}\left(F_{o}{ }^{2}\right)+\left(m^{*} p\right)^{2}+n * p\right], p=\left[\max \left(F_{o}{ }^{2}, 0\right)+2^{*} F_{c}{ }^{2}\right] / 3, m$ \& $n$ are constants. 
Table 2. Crystal data and structure refinement for (R)-4c (CCDC Code: 2088284).

\begin{tabular}{|c|c|c|}
\hline Empirical formula & \multicolumn{2}{|l|}{$\mathrm{C}_{7} \mathrm{H}_{12} \mathrm{ClNO}_{3}$} \\
\hline Formula weight & \multicolumn{2}{|l|}{193.63} \\
\hline Temperature & \multicolumn{2}{|l|}{$100(2) \mathrm{K}$} \\
\hline Wavelength & \multicolumn{2}{|l|}{$0.71073 \AA$} \\
\hline Crystal system & \multicolumn{2}{|l|}{ Orthorhombic } \\
\hline Space group & \multicolumn{2}{|l|}{$\mathrm{P} 2{ }_{1} 2_{1} 2_{1}$} \\
\hline \multirow[t]{3}{*}{ Unit cell dimensions } & $\mathrm{a}=8.4411(3) \AA$ & $\alpha=90^{\circ}$. \\
\hline & $b=9.5203(3) \AA$ & $\beta=90^{\circ}$. \\
\hline & $c=12.3234(5) \AA$ & $\gamma=90^{\circ}$ \\
\hline Volume & \multicolumn{2}{|l|}{$990.33(6) \AA^{3}$} \\
\hline $\mathrm{Z}$ & \multicolumn{2}{|l|}{4} \\
\hline Density (calculated) & \multicolumn{2}{|l|}{$1.299 \mathrm{Mg} / \mathrm{m}^{3}$} \\
\hline Absorption coefficient & \multicolumn{2}{|l|}{$0.357 \mathrm{~mm}^{-1}$} \\
\hline $\mathrm{F}(000)$ & \multicolumn{2}{|l|}{408} \\
\hline Crystal size & \multicolumn{2}{|c|}{$0.25 \times 0.24 \times 0.13 \mathrm{~mm}^{3}$} \\
\hline Theta range for data collection & \multicolumn{2}{|l|}{2.704 to $27.499^{\circ}$. } \\
\hline Index ranges & \multicolumn{2}{|c|}{$-10<=\mathrm{h}<=6,-12<=\mathrm{k}<=5,-10<=1<=16$} \\
\hline Reflections collected & \multicolumn{2}{|l|}{3733} \\
\hline Independent reflections & \multicolumn{2}{|c|}{$2085[\mathrm{R}(\mathrm{int})=0.0183]$} \\
\hline Completeness to theta $=25.242^{\circ}$ & \multicolumn{2}{|l|}{$99.7 \%$} \\
\hline Refinement method & \multicolumn{2}{|c|}{ Full-matrix least-squares on $\mathrm{F}^{2}$} \\
\hline Data / restraints / parameters & \multicolumn{2}{|l|}{2085 / 0 / 112} \\
\hline Goodness-of-fit on $\mathrm{F}^{2}$ & \multicolumn{2}{|l|}{1.066} \\
\hline
\end{tabular}


Final R indices [I>2sigma(I)]

$\mathrm{R}$ indices (all data)

Absolute structure parameter

Extinction coefficient

Largest diff. peak and hole
$\mathrm{R} 1=0.0291, \mathrm{wR} 2=0.0717$

$\mathrm{R} 1=0.0315, \mathrm{wR} 2=0.0728$

$0.23(5)$

$0.000(2)$

0.245 and -0.209 e. $\AA^{-3}$

$\mathrm{R} 1=\sum\left(|| \mathrm{Fo}_{\mathrm{O}}|-| \mathrm{F}_{\mathrm{C}} \mid\right) / \sum\left|\mathrm{Fo}_{\mathrm{o}}\right| \quad \mathrm{WR2}=\left[\sum\left[\mathrm{w}\left(\mathrm{Fo}_{\mathrm{o}}{ }^{2}-\mathrm{F}_{\mathrm{c}}{ }^{2}\right)^{2}\right] / \sum\left[\mathrm{w}\left(\mathrm{F}_{\mathrm{o}}{ }^{2}\right)^{2}\right]\right]^{1 / 2}$

$S=\left[\sum\left[w\left(F_{o}{ }^{2}-F_{c}{ }^{2}\right)^{2}\right] /(n-p)\right]^{1 / 2} w=1 /\left[\sigma^{2}\left(F_{o}{ }^{2}\right)+\left(m^{*} p\right)^{2}+n * p\right], p=\left[\max \left(F_{o}{ }^{2}, 0\right)+2^{*} F_{c}{ }^{2}\right] / 3, m \& n$ are constants. 
Table 3. Crystal data and structure refinement for (R)-10b (CCDC Code: 2088285).

Empirical formula

Formula weight

Temperature

Wavelength

Crystal system

Space group

Unit cell dimensions

Volume

Z

Density (calculated)

Absorption coefficient

$\mathrm{F}(000)$

Crystal size

Theta range for data collection

Index ranges

Reflections collected

Independent reflections

Completeness to theta $=67.679^{\circ}$

Absorption correction

Refinement method

Data / restraints / parameters

Goodness-of-fit on $\mathrm{F}^{2}$

Final R indices [I $>2 \operatorname{sigma}(\mathrm{I})]$
$\mathrm{C}_{15} \mathrm{H}_{15} \mathrm{~N}_{2} \mathrm{O}_{5}$

303.29

100(2) K

$1.54178 \AA$

Monoclinic

$\mathrm{P} 2_{1}$

$$
\begin{array}{ll}
\mathrm{a}=9.9449(3) \AA & \alpha=90^{\circ} \\
\mathrm{b}=4.98240(10) \AA & \beta=93.5340(10)^{\circ} \\
\mathrm{c}=14.8804(4) \AA & \gamma=90^{\circ}
\end{array}
$$

735.91(3) $\AA^{3}$

2

$1.369 \mathrm{Mg} / \mathrm{m}^{3}$

$0.876 \mathrm{~mm}^{-1}$

318

$0.251 \times 0.058 \times 0.050 \mathrm{~mm}^{3}$

2.975 to $78.909^{\circ}$.

$-12 \leq \mathrm{h} \leq 12,-6 \leq \mathrm{k} \leq 5,-18 \leq \mathrm{l} \leq 18$

49409

$3042[\mathrm{R}$ (int) $=0.0307]$

$97.7 \%$

None

Full-matrix least-squares on $\mathrm{F}^{2}$

3042 / 1 / 205

1.061

$\mathrm{R} 1=0.0259, \mathrm{wR} 2=0.0668[3001]$ 
$\mathrm{R}$ indices (all data)

Absolute structure parameter

Largest diff. peak and hole
$\mathrm{R} 1=0.0263, \mathrm{wR} 2=0.0670$

$0.04(4)$

0.182 and -0.136 e. $\AA^{-3}$

$\mathrm{R} 1=\sum\left(|| \mathrm{Fo}_{\mathrm{o}}|-| \mathrm{F}_{\mathrm{c}}||\right) / \sum\left|\mathrm{Fo}_{\mathrm{o}}\right| \mathrm{wR} 2=\left[\sum\left[\mathrm{w}\left(\mathrm{Fo}_{\mathrm{o}}^{2}-\mathrm{Fc}^{2}\right)^{2}\right] / \sum\left[\mathrm{w}\left(\mathrm{Fo}_{\mathrm{o}}^{2}\right)^{2}\right]\right]^{1 / 2}$

$S=\left[\sum\left[w\left(F_{o}{ }^{2}-F_{c}{ }^{2}\right)^{2}\right] /(n-p)\right]^{1 / 2} w=1 /\left[\sigma^{2}\left(F_{o}{ }^{2}\right)+(m * p)^{2}+n * p\right], p=\left[\max \left(F_{o}{ }^{2}, 0\right)+2^{*} F_{c}{ }^{2}\right] / 3, m$ \& $n$ are constants. 